TTP07-19

SFB/CPP-07-43

MPP-2007-102

DESY 07-112

\title{
Electroweak corrections to hadronic production of $W$ bosons at large transverse momenta
}

\author{
Johann H. KÜHN ${ }^{a}$, A. Kulesza ${ }^{b}$, S. Pozzorini ${ }^{c}$, M. Schulze ${ }^{a}$ \\ ${ }^{a}$ Institut für Theoretische Teilchenphysik, Universität Karlsruhe, \\ D-76128 Karlsruhe, Germany \\ ${ }^{b}$ Deutsches Elektronen-Synchrotron DESY, Notkestrasse 85, \\ D-22607 Hamburg, Germany \\ ${ }^{c}$ Max-Planck-Institut für Physik, Föhringer Ring 6, \\ D-80805 Munich, Germany
}

\begin{abstract}
:
To match the precision of present and future measurements of $W$-boson production at hadron colliders electroweak radiative corrections must be included in the theory predictions. In this paper we consider their effect on the transverse momentum $\left(p_{\mathrm{T}}\right)$ distribution of $W$ bosons, with emphasis on large $p_{\mathrm{T}}$. We evaluate the full electroweak $\mathcal{O}(\alpha)$ corrections to the processes $p p \rightarrow W+$ jet and $p \bar{p} \rightarrow W+$ jet including virtual and real photonic contributions. We present the explicit expressions in analytical form for the virtual corrections and provide results for the real corrections, discussing in detail the treatment of soft and collinear singularities. We also provide compact approximate expressions which are valid in the high-energy region, where the electroweak corrections are strongly enhanced by logarithms of $\hat{s} / M_{W}^{2}$. These expressions describe the complete asymptotic behaviour at one loop as well as the leading and next-to-leading logarithms at two loops. Numerical results are presented for proton-proton collisions at $14 \mathrm{TeV}$ and proton-antiproton collisions at $2 \mathrm{TeV}$. The corrections are negative and their size increases with $p_{\mathrm{T}}$. At the LHC, where transverse momenta of $2 \mathrm{TeV}$ or more can be reached, the one- and two-loop corrections amount up to $-40 \%$ and $+10 \%$, respectively, and will be important for a precise analysis of $W$ production. At the Tevatron, transverse momenta up to $300 \mathrm{GeV}$ are within reach. In this case the electroweak corrections amount up to $-10 \%$ and are thus larger than the expected statistical error.
\end{abstract}




\section{Introduction}

After the startup of the Large Hadron Collider (LHC) hard scattering reactions will be explored with high event rates and momentum transfers up to several $\mathrm{TeV}$. In order to identify new phenomena in this region, the predictions of the Standard Model have to be understood with adequate precision.

The study of gauge-boson production has been among the primary goals of hadron colliders, starting with the discovery of the $W$ and $Z$ bosons more than two decades ago [1]. The investigation of the production dynamics, strictly predicted by the electroweak theory, constitutes one of the important tests of the Standard Model. Differential distributions of gauge bosons, in rapidity as well as in transverse momentum $\left(p_{\mathrm{T}}\right)$, have always been the subject of theoretical and experimental studies. This allows to search for and set limits on anomalous gauge-boson couplings, measure the parton distribution functions and, if understood sufficiently well, use these reactions to calibrate the luminosity. For gauge-boson production at large $p_{\mathrm{T}}$ the final state of the leading-order process consists of an electroweak gauge boson plus one recoiling jet. Being, in leading order, proportional to the strong coupling constant, these reactions could also lead to a determination of $\alpha_{\mathrm{S}}$ in the $\mathrm{TeV}$ region.

The high center-of-mass energy at the LHC in combination with its enormous luminosity will allow to produce gauge bosons with transverse momenta up to $2 \mathrm{TeV}$ or even beyond. In this kinematic region the electroweak corrections are strongly enhanced, with the dominant terms in $L$-loop approximation being leading logarithms (LL) of the form $\alpha^{L} \log ^{2 L}\left(\hat{s} / M_{W}^{2}\right)$, next-to-leading logarithms (NLL) of the form $\alpha^{L} \log ^{2 L-1}\left(\hat{s} / M_{W}^{2}\right)$, and so on. These corrections, also known as electroweak Sudakov logarithms, may well amount to several tens of percent [2, 3, 4, 15, 6, 7, , 8, 9, 10, 11]. (A recent survey of the literature on electroweak Sudakov logarithms can be found in Ref. [12].) Specifically, the electroweak corrections to the $p_{\mathrm{T}}$-distribution of photons and $Z$ bosons at hadron colliders were studied in Refs. [7, 8, , 9, 10]. In Refs. [8, 19, 10, it was found that at transverse momenta of $\mathcal{O}(1 \mathrm{TeV})$ the dominant two-loop contributions to these reactions amount to several percent and must be included to match the precision of the LHC experiments. This is quite different from the production of on-shell gauge bosons with small transverse momenta [13], where the electroweak corrections are not enhanced by Sudakov logarithms. With this motivation in mind we study the electroweak corrections to hadronic production of $W$ bosons in association with a jet, $p\left(p^{-}\right) \rightarrow W j$, at large $p_{\mathrm{T}}$.

As a consequence of the non-vanishing $W$ charge, QED corrections cannot be separated from the purely weak ones and will thus be included in our analysis. Thus, in comparison with $Z$-boson production, several new aspects arise. Real photon emission must be included to cancel the infrared divergencies from virtual photonic corrections. Collinear singularities, a consequence of radiation from massless quarks, must be isolated and absorbed in the parton distribution functions (PDFs) in the case of initial-state radiation. We regularize soft and collinear singularities in two different schemes: using small quark and photon masses which are set to zero at the 
end of the calculation and, alternatively, dimensional regularization. In events with real radiation, the $p_{\mathrm{T}}$ of the $W$ boson is balanced both by the $p_{\mathrm{T}}$ of the recoiling parton (quark or gluon) and the photon. Configurations involving a small- $p_{\mathrm{T}}$ parton and a hard photon are better described as $W \gamma$ final states. We thus define the $W j$ cross section imposing a lower limit on the jet transverse momentum, which is chosen independent of the $W$-boson $p_{\mathrm{T}}$. In order to avoid final-state collinear singularities, we recombine collinear photon-quark final states.

The virtual EW corrections to $W j$ production are formally connected with the real emission of $W$ and $Z$ bosons, which leads to $W V j$ final states with $V=W, Z$. Both contributions are of $\mathcal{O}\left(\alpha^{2} \alpha_{\mathrm{S}}\right)$. If integrated over the full phase space, the real emission of gauge bosons produces large Sudakov logarithms that partially cancel those resulting from virtual gauge bosons. However, in exclusive measurements of $p p \rightarrow W j$, the available phase space for gauge boson emission is strongly suppressed by the experimental cuts. We thus expect that real emission provides relatively small contributions while the bulk of electroweak effects originates from virtual corrections. In fact, for $p p \rightarrow Z j$ it was shown that, in presence of realistic (and relatively less exclusive) experimental cuts, the contribution of real emission is about five times smaller than the virtual corrections [11]. Moreover, real emission can be further reduced with a veto on additional jets, which suppresses multiple-jet events resulting from the hadronic decay of the radiated gauge bosons. Therefore we will restrict ourselves to the investigation of virtual electroweak corrections (and photon bremsstrahlung). The real emission of $W$ and $Z$ bosons can be non-negligible and certainly deserves further detailed studies, however we do not expect a dramatic impact on our results.

The partonic reactions $\bar{q} q^{\prime} \rightarrow W^{ \pm} g(\gamma), q^{\prime} g \rightarrow W^{ \pm} q(\gamma)$ and $\bar{q} g \rightarrow W^{ \pm} \bar{q}^{\prime}(\gamma)$ with $q=u, d, s, c, b$ are considered. All of them are, however, trivially related by $\mathrm{CP}$ - and crossing-symmetry relations. Quark-mass effects are neglected throughout, which allows to incorporate the effect of quark mixing through a simple redefinition of parton distribution functions (see Sect. 2.1). Our conventions for couplings, kinematics and two- as well as three-body phase space are also collected in Sect. 2. The calculation of the virtual corrections is described in Sect. 3. We present analytic expressions for the one-loop amplitude, specify the counterterms in the $G_{\mu}$ renormalization scheme and isolate the infrared singularities. The high-energy limit is studied in detail in Sect. 4. The analytic one-loop result is investigated in the limit $\hat{s} \gg M_{W}^{2}$, keeping quadratic and linear logarithms as well as constant terms. These results are compared to those derived in the NLL approximation [14]. In view of their numerical importance we also derive the dominant (NLL) two-loop terms, using the formalism of Refs. [15, 16]. The calculation of the real corrections is performed using the dipole subtraction formalism [17, 18, 19], as discussed in Sect. 5. The checks which we carried out in order to ensure the correctness of the results are described in Sect. 6.

The numerical results are presented in Sect. 7. After convolution with parton distribution functions, we obtain radiatively corrected predictions for $p_{\mathrm{T}^{-}}$-distributions 
of $W$ bosons at the LHC and the Tevatron. The quality of the one-loop NLL and NNLL approximations is investigated and the size of the dominant two-loop terms is compared with the expected statistical precision of the experiments. Concerning perturbative QCD, our predictions are based on the lowest order. To obtain realistic absolute cross sections, higher-order QCD corrections [20] must be included. However, the relative rates for $W^{+}, W^{-}$and $Z$ production are expected to be more stable against QCD effects. Therefore, the impact of the electroweak corrections on these ratios is presented in Sect. 7. Our conclusions and a brief summary can be found in Sect. 8. Explicit analytic results are collected in the Appendices.

A short description of the method of calculation and the main results for LHC have been given in Ref. [21]. After completion of this work, Hollik, Kasprzik and Kniehl [22] reported results on hadronic $W$-boson production at large $p_{\mathrm{T}}$ qualitatively similar to those of Ref. [21] and the present paper.

\section{Definitions and conventions}

\subsection{Hadronic cross section}

The $p_{\mathrm{T}}$-distribution of $W$ bosons in the reaction $h_{1} h_{2} \rightarrow W^{ \pm} j(\gamma)$ is given by

$$
\frac{\mathrm{d} \sigma^{h_{1} h_{2}}}{\mathrm{~d} p_{\mathrm{T}}}=\sum_{a, b, k} \int_{0}^{1} \mathrm{~d} x_{1} \int_{0}^{1} \mathrm{~d} x_{2} \theta\left(x_{1} x_{2}-\hat{\tau}_{\min }\right) f_{h_{1}, a}\left(x_{1}, \mu^{2}\right) f_{h_{2}, b}\left(x_{2}, \mu^{2}\right) \frac{\mathrm{d} \hat{\sigma}^{a b \rightarrow W^{\sigma} k(\gamma)}}{\mathrm{d} p_{\mathrm{T}}}
$$

where $\hat{\tau}_{\text {min }}$ depends on the kinematic configuration of the final state and is specified at the end of Sect. 2.2. The indices $a, b$ denote initial-state partons and $f_{h_{1}, a}\left(x, \mu^{2}\right)$, $f_{h_{2}, b}\left(x, \mu^{2}\right)$ are the corresponding parton distribution functions (PDFs). $\hat{\sigma}^{a b \rightarrow W^{\sigma} k(\gamma)}$ is the partonic cross section for the subprocess $a b \rightarrow W^{\sigma} k(\gamma)$. The sum in (1) runs over all $a, b, k$ combinations corresponding to the subprocesses

$$
\begin{array}{lll}
\bar{d}_{n} u_{m} \rightarrow W^{+} g(\gamma), & u_{m} \bar{d}_{n} \rightarrow W^{+} g(\gamma), & g u_{m} \rightarrow W^{+} d_{n}(\gamma), \\
u_{m} g \rightarrow W^{+} d_{n}(\gamma), & \bar{d}_{n} g \rightarrow W^{+} \bar{u}_{m}(\gamma), & g \bar{d}_{n} \rightarrow W^{+} \bar{u}_{m}(\gamma),
\end{array}
$$

for $W^{+}$production, and similarly for $W^{-}$production.

The dependence of the partonic cross sections on the family indices $m, n$ amounts to an overall factor $\left|V_{u_{m} d_{n}}\right|^{2}$. This factor can be easily absorbed by redefining the parton distribution functions as

$$
\begin{aligned}
& \tilde{f}_{h, d_{m}}=\sum_{n=1}^{3}\left|V_{u_{m} d_{n}}\right|^{2} f_{h, d_{n}}, \quad \tilde{f}_{h, \bar{d}_{m}}=\sum_{n=1}^{3}\left|V_{u_{m} d_{n}}\right|^{2} f_{h, \bar{d}_{n}}, \\
& \tilde{f}_{h, u_{m}}=f_{h, u_{m}}, \quad \tilde{f}_{h, \bar{u}_{m}}=f_{h, \bar{u}_{m}}, \quad \tilde{f}_{h, g}=f_{h, g} .
\end{aligned}
$$

The hadronic cross section (11) can be computed using the trivial CKM matrix $\tilde{V}_{u_{i} d_{j}}=\delta_{i j}$ and the redefined PDFs (3). Since we do not consider initial or final 
states involving (anti-)top quarks, only the contributions of the first two quark families $(m=1,2)$ have to be included. The corresponding redefined $\operatorname{PDFs}\left(\tilde{f}_{h, q}\right.$ with $q=u, d, c, s)$ automatically include the (small) contributions associated with initial- and final-state bottom quarks.

\section{$2.2 \quad$ Kinematics}

For the $2 \rightarrow 2$ subprocess $a b \rightarrow W^{\sigma} k$ the Mandelstam variables are defined in the standard way,

$$
\hat{s}=\left(p_{a}+p_{b}\right)^{2}, \quad \hat{t}=\left(p_{a}-p_{W}\right)^{2}, \quad \hat{u}=\left(p_{b}-p_{W}\right)^{2} .
$$

The momenta $p_{a}, p_{b}, p_{k}$ of the partons are assumed to be massless, whereas $p_{W}^{2}=$ $M_{W}^{2}$. In terms of $x_{1}, x_{2}, p_{\mathrm{T}}$ and the collider energy $\sqrt{s}$ we have

$$
\hat{s}=x_{1} x_{2} s, \quad \hat{t}=\frac{M_{W}^{2}-\hat{s}}{2}(1-\cos \theta), \quad \hat{u}=\frac{M_{W}^{2}-\hat{s}}{2}(1+\cos \theta),
$$

with $\cos \theta=\sqrt{1-4 p_{T}^{2} \hat{s} /\left(\hat{s}-M_{W}^{2}\right)^{2}}$ corresponding to the cosine of the angle between the momenta $p_{a}$ and $p_{W}$ in the partonic center-of-mass frame.

The $p_{\mathrm{T}}$-distribution for the unpolarized partonic subprocess $a b \rightarrow W^{\sigma} k$ reads

$$
\frac{\mathrm{d} \hat{\sigma}^{a b \rightarrow W^{\sigma} k}}{\mathrm{~d} p_{\mathrm{T}}}=\mathcal{N}_{a b} \int \mathrm{d} \Phi_{2} \bar{\sum}\left|\mathcal{M}^{a b \rightarrow W^{\sigma} k}\right|^{2} F_{\mathrm{O}, 2}\left(\Phi_{2}\right),
$$

where $\bar{\sum}=\frac{1}{4} \sum_{\mathrm{pol}} \sum_{\mathrm{col}}$ involves the sum over polarization and color as well as the average factor $1 / 4$ for initial-state polarization. The factor $\mathcal{N}_{a b}$ is given by

$$
\mathcal{N}_{a b}=\frac{(2 \pi)^{4}}{2 \hat{s} N_{a b}}
$$

where $N_{\bar{q} q^{\prime}}=N_{q \bar{q}^{\prime}}=N_{\mathrm{c}}^{2}, N_{g q}=N_{q g}=N_{\bar{q} g}=N_{g \bar{q}}=N_{\mathrm{c}}\left(N_{\mathrm{c}}^{2}-1\right)$, with $N_{\mathrm{c}}=3$, account for the initial-state colour average. The phase-space measure $d \Phi_{2}$ is given by

$$
\mathrm{d} \Phi_{2}=\frac{\mathrm{d}^{3} p_{W}}{(2 \pi)^{3} 2 p_{W}^{0}} \frac{\mathrm{d}^{3} p_{k}}{(2 \pi)^{3} 2 p_{k}^{0}} \delta^{4}\left(p_{a}+p_{b}-p_{W}-p_{k}\right),
$$

while the function $F_{\mathrm{O}, 2}$ defines the observable of interest, i.e. the $W$-boson $p_{\mathrm{T}^{-}}$ distribution in presence of a cut on the transverse momentum of the jet,

$$
F_{\mathrm{O}, 2}\left(\Phi_{2}\right)=\delta\left(p_{\mathrm{T}}-p_{\mathrm{T}, W}\right) \theta\left(p_{\mathrm{T}, j}-p_{\mathrm{T}, j}^{\min }\right)
$$

In the 2-particle phase space the jet is identified with the parton $k$ and momentum conservation implies $p_{\mathrm{T}, j}=p_{\mathrm{T}, k}=p_{\mathrm{T}, W}$. In practice, since we always consider the $p_{\mathrm{T}}$-distribution in the region $p_{\mathrm{T}}>p_{\mathrm{T}, W}^{\min }>p_{\mathrm{T}, j}^{\min }$, the cut on $p_{\mathrm{T}, j}$ in (9) is irrelevant. The phase-space integral in (6) yields two contributions originating from kinematic 
configurations in the forward and backward hemispheres with opposite values of $\cos \theta$ in the center-of-mass frame,

$$
\frac{\mathrm{d} \hat{\sigma}^{a b \rightarrow W^{\sigma} k}}{\mathrm{~d} p_{\mathrm{T}}}=\frac{\mathrm{d} \hat{\sigma}_{\mathrm{fwd}}^{a b \rightarrow W^{\sigma} k}}{\mathrm{~d} p_{\mathrm{T}}}+\frac{\mathrm{d} \hat{\sigma}_{\mathrm{bkwd}}^{a b \rightarrow W^{\sigma} k}}{\mathrm{~d} p_{\mathrm{T}}}
$$

with

$$
\frac{\mathrm{d} \hat{\sigma}_{\mathrm{fwd}}^{a b \rightarrow W^{\sigma} k}}{\mathrm{~d} p_{\mathrm{T}}}=\frac{p_{\mathrm{T}}}{8 \pi N_{a b} \hat{s}|\hat{t}-\hat{u}|} \bar{\sum}\left|\mathcal{M}^{a b \rightarrow W^{\sigma} k}\right|^{2}, \quad \frac{\mathrm{d} \hat{\sigma}_{\mathrm{bkwd}}^{a b \rightarrow W^{\sigma} k}}{\mathrm{~d} p_{\mathrm{T}}}=\left.\frac{\mathrm{d} \hat{\sigma}_{\mathrm{fwd}}^{a b \rightarrow W^{\sigma} k}}{\mathrm{~d} p_{\mathrm{T}}}\right|_{\hat{t} \leftrightarrow \hat{u}} .
$$

For the $2 \rightarrow 3$ subprocess $a b \rightarrow W^{\sigma} k \gamma$ we define the following five independent invariants

$$
\begin{aligned}
& \hat{s}=\left(p_{a}+p_{b}\right)^{2}, \quad \hat{t}=\left(p_{a}-p_{W}\right)^{2}, \quad \hat{u}=\left(p_{b}-p_{W}\right)^{2}, \\
& \hat{t}^{\prime}=\left(p_{a}-p_{\gamma}\right)^{2}, \quad \hat{u}^{\prime}=\left(p_{b}-p_{\gamma}\right)^{2},
\end{aligned}
$$

and the four dependent invariants

$$
\begin{aligned}
\hat{s}^{\prime}=\left(p_{k}+p_{\gamma}\right)^{2}=\hat{s}+\hat{t}+\hat{u}-M_{W}^{2}, & \hat{s}^{\prime \prime}=\left(p_{W}+p_{k}\right)^{2}=\hat{s}+\hat{t}^{\prime}+\hat{u}^{\prime}, \\
\hat{t}^{\prime \prime}=\left(p_{a}-p_{k}\right)^{2}=M_{W}^{2}-\hat{s}-\hat{t}-\hat{t}^{\prime}, & \hat{u}^{\prime \prime}=\left(p_{b}-p_{k}\right)^{2}=M_{W}^{2}-\hat{s}-\hat{u}-\hat{u}^{\prime} .
\end{aligned}
$$

The $p_{\mathrm{T}}$-distribution for this subprocess reads

$$
\frac{\mathrm{d} \hat{\sigma}^{a b \rightarrow W^{\sigma} k \gamma}}{\mathrm{d} p_{\mathrm{T}}}=\mathcal{N}_{a b} \int \mathrm{d} \Phi_{3} \bar{\sum}\left|\mathcal{M}^{a b \rightarrow W^{\sigma} k \gamma}\right|^{2} F_{\mathrm{O}, 3}\left(\Phi_{3}\right),
$$

where

$$
\mathrm{d} \Phi_{3}=\frac{\mathrm{d}^{3} p_{W}}{(2 \pi)^{3} 2 p_{W}^{0}} \frac{\mathrm{d}^{3} p_{k}}{(2 \pi)^{3} 2 p_{k}^{0}} \frac{\mathrm{d}^{3} p_{\gamma}}{(2 \pi)^{3} 2 p_{\gamma}^{0}} \delta^{4}\left(p_{a}+p_{b}-p_{W}-p_{k}-p_{\gamma}\right) .
$$

In the 3 -particle phase space, the $W$-boson $p_{\mathrm{T}}$-distribution in $W j$ production is defined by the observable function

$$
F_{\mathrm{O}, 3}\left(\Phi_{3}\right)=\delta\left(p_{\mathrm{T}}-p_{\mathrm{T}, W}\right) \theta\left(p_{\mathrm{T}, j}-p_{\mathrm{T}, j}^{\min }\right)
$$

The cut on the jet transverse momentum rejects events where the $W$-boson $p_{\mathrm{T}}$ is balanced by an isolated photon plus a parton with small transverse momentum. This observable is thus free from singularities associated with soft and collinear quarks or gluons. When applying the cut on the jet momentum in the 3-particle phase space, care must be taken that the definition of the jet $p_{\mathrm{T}}$ is collinear-safe. In general the jet cannot be identified with the parton $k$, since in presence of collinear photon radiation the transverse momentum of a charged parton is not a collinear-safe quantity. Thus we identify the jet with the parton $k$ only if $k$ is a quark well separated from the 
photon or a gluon. Otherwise, i.e. for collinear quark-photon configurations, the recombined momentum of the quark and photon is taken as momentum of the jet. In practice, we define the separation variable

$$
R(q, \gamma)=\sqrt{\left(\eta_{q}-\eta_{\gamma}\right)^{2}+\left(\phi_{q}-\phi_{\gamma}\right)^{2}}
$$

where $\eta_{i}$ is the pseudo-rapidity and $\phi_{i}$ is the azimuthal angle of a particle $i$. If $R(q, \gamma)<R_{\text {sep }}$, then the photon and quark momenta are recombined by simple four-vector addition into an effective momentum $p_{j}$ and then $p_{\mathrm{T}, j}=\sqrt{\left(\vec{p}_{\mathrm{T}, q}+\vec{p}_{\mathrm{T}, \gamma}\right)^{2}}$, otherwise $p_{\mathrm{T}, j}=p_{\mathrm{T}, q}$. We note that, in the collinear region, lowest-order kinematics implies $p_{\mathrm{T}, j}=p_{\mathrm{T}, q}+p_{\mathrm{T}, \gamma}=p_{\mathrm{T}, W}>p_{\mathrm{T}, j}^{\min }$. This means that the recombination procedure effectively removes the cut on $p_{\mathrm{T}, q}$ inside the collinear cone $R(q, \gamma)<R_{\mathrm{sep}}$. For instance the recombined $g q^{\prime} \rightarrow W^{\sigma} q \gamma$ cross section is given by

$$
\hat{\sigma}_{\text {rec. }}^{g q^{\prime} \rightarrow W^{\sigma} q \gamma}=\int_{R(q, \gamma)<R_{\text {sep }}} \mathrm{d} \hat{\sigma}^{g q^{\prime} \rightarrow W^{\sigma} q \gamma}+\int_{R(q, \gamma)>R_{\text {sep }}} \theta\left(p_{\mathrm{T}, q}-p_{\mathrm{T}, j}^{\min }\right) \mathrm{d} \hat{\sigma}^{g q^{\prime} \rightarrow W^{\sigma} q \gamma} .(18
$$

In contrast, for the case of final-state gluons, we do not perform photon-gluon recombination and the cut on $p_{\mathrm{T}, g}$ is imposed in the entire phase space.

This procedure has the advantage to avoid both collinear-photon and soft-gluon singularities. However it implies a different treatment of quark and gluon final states and can thus be regarded as an arbitrary cut-off prescription for the final-state collinear singularity. Moreover, the recombined cross section (18) has a logarithmic dependence on the cut-off parameter $R_{\text {sep }}$. These aspects are discussed in detail in Appendix A. There we compare the recombination procedure with a realistic experimental definition of exclusive $p p \rightarrow W j$ production, where final-state quarks are subject to the same cut as final state gluons $\left(p_{\mathrm{T}, q}>p_{\mathrm{T}, j}^{\min }\right)$ within the entire phase space. Describing the exclusive $g q^{\prime} \rightarrow W^{\sigma} q \gamma$ cross section,

$$
\hat{\sigma}_{\text {excl. }}^{g q^{\prime} \rightarrow W^{\sigma} q \gamma}=\int \theta\left(p_{\mathrm{T}, q}-p_{\mathrm{T}, j}^{\min }\right) \mathrm{d} \hat{\sigma}^{g q^{\prime} \rightarrow W^{\sigma} q \gamma},
$$

by means of quark fragmentation functions, we find that the quantitative difference between the two definitions (18) and (19) amounts to less than two permille. Moreover, we show that the recombined cross section is extremely stable with respect to variations of the parameter $R_{\mathrm{sep}}$. This means that the recombination procedure used in our calculation provides a very good description of exclusive $p p \rightarrow W j$ production.

Another treatment of the singularities, which does not require recombination and treats quark- and gluon-induced jets uniformly, has been proposed in Ref. [22]. There, contributions from $W j$ production and $W \gamma$ production to a more inclusive observable, i.e. high- $p_{\mathrm{T}} W$ production, are both calculated. All soft and collinear singularities in the final state cancel in the approach of Ref. [22] as a result of the more inclusive observable definition than associated production of the $W$ boson together with a jet, considered in this work. The comparison of our results with 
those of Ref. [22] seems to indicate that these differences in the jet definitions have a quite small impact on the size of the electroweak corrections.

The quantity $\hat{\tau}_{\text {min }}$ in (11) is related to the minimum partonic energy that is needed to produce final states with $p_{\mathrm{T}, j}>p_{\mathrm{T}, j}^{\min }$ and $p_{\mathrm{T}, W}>p_{\mathrm{T}, W}^{\min }$,

$$
s \hat{\tau}_{\min }=\left(p_{\mathrm{T}, j}^{\min }+\sqrt{\left(p_{\mathrm{T}, W}^{\min }\right)^{2}+M_{W}^{2}}\right)^{2} .
$$

When we evaluate the $2 \rightarrow 2$ contributions to the hadronic cross section (11), after analytic integration of the phase space in (6) , we can set $p_{\mathrm{T}, j}^{\min }=p_{\mathrm{T}, W}^{\min }=p_{\mathrm{T}}$ in (20) .

\subsection{Crossing symmetries}

The unpolarized squared matrix elements for the $2 \rightarrow 2$ processes in (2) are related by the crossing-symmetry relations

$$
\begin{aligned}
& \bar{\sum}\left|\mathcal{M}^{g q^{\prime} \rightarrow W^{\sigma} q}\right|^{2}=-\left.\bar{\sum}\left|\mathcal{M}^{\bar{q} q^{\prime} \rightarrow W^{\sigma} g}\right|^{2}\right|_{\hat{s} \leftrightarrow \hat{t}}, \\
& \bar{\sum}\left|\mathcal{M}^{\bar{q} g \rightarrow W^{\sigma} \bar{q}^{\prime}}\right|^{2}=-\left.\bar{\sum}\left|\mathcal{M}^{\bar{q} q^{\prime} \rightarrow W^{\sigma} g}\right|^{2}\right|_{\hat{s} \leftrightarrow \hat{u}}, \\
& \bar{\sum}\left|\mathcal{M}^{b a \rightarrow W^{\sigma} k}\right|^{2}=\left.\bar{\sum}\left|\mathcal{M}^{a b \rightarrow W^{\sigma} k}\right|^{2}\right|_{\hat{t} \leftrightarrow \hat{u}} .
\end{aligned}
$$

Moreover, due to CP symmetry, the unpolarized partonic cross section for the production of positively and negatively charged $W$ bosons are related by

$$
\bar{\sum}\left|\mathcal{M}^{\bar{d} u \rightarrow W^{+} g}\right|^{2}=\bar{\sum}\left|\mathcal{M}^{d \bar{u} \rightarrow W^{-} g}\right|^{2} .
$$

Eqs. (21) and (22) permit to relate the six processes for $W^{+}$production in (2) and the six charge conjugate ones to a single process. Hence the explicit computation of the unpolarized squared matrix element needs to be performed only once. In the following we will present explicit results for the process $\bar{q} q^{\prime} \rightarrow W^{\sigma} g$.

Similarly, for the unpolarized squared matrix elements for the $2 \rightarrow 3$ processes in (2) we have

$$
\begin{aligned}
& \bar{\sum}\left|\mathcal{M}^{g q^{\prime} \rightarrow W^{\sigma} q \gamma}\right|^{2}=-\left.\bar{\sum}\left|\mathcal{M}^{\bar{q} q^{\prime} \rightarrow W^{\sigma} g \gamma}\right|^{2}\right|_{\left\{\hat{s} \leftrightarrow \hat{u}^{\prime \prime}, \hat{t} \leftrightarrow \hat{s}^{\prime \prime}, \hat{t}^{\prime} \leftrightarrow \hat{s}^{\prime}\right\}}, \\
& \bar{\sum}\left|\mathcal{M}^{\bar{q} g \rightarrow W^{\sigma} \bar{q}^{\prime} \gamma}\right|^{2}=-\left.\bar{\sum}\left|\mathcal{M}^{\bar{q} q^{\prime} \rightarrow W^{\sigma} g \gamma}\right|^{2}\right|_{\left\{\hat{s} \leftrightarrow \hat{t}^{\prime \prime}, \hat{u} \leftrightarrow \hat{s}^{\prime \prime}, \hat{u}^{\prime} \leftrightarrow \hat{s}^{\prime}\right\}}, \\
& \bar{\sum}\left|\mathcal{M}^{b a \rightarrow W^{\sigma} k \gamma}\right|^{2}=\left.\bar{\sum}\left|\mathcal{M}^{a b \rightarrow W^{\sigma} k \gamma}\right|^{2}\right|_{\left\{\hat{t} \leftrightarrow \hat{u}, \hat{t}^{\prime} \leftrightarrow \hat{u}^{\prime}\right\}}
\end{aligned}
$$

and

$$
\bar{\sum}\left|\mathcal{M}^{\bar{d} u \rightarrow W^{+} g \gamma}\right|^{2}=\bar{\sum}\left|\mathcal{M}^{d \bar{u} \rightarrow W^{-} g \gamma}\right|^{2} .
$$

It is thus enough to perform calculations only for the $\bar{q} q^{\prime} \rightarrow W^{\sigma} g \gamma$ subprocess. 


\subsection{Couplings and Born matrix element}

For gauge couplings we adopt the conventions of Ref. [23]. With this notation the $g q \bar{q}$ vertex and the $V q^{\prime} \bar{q}$ vertices with $V=A, Z, W^{ \pm} \operatorname{read}$
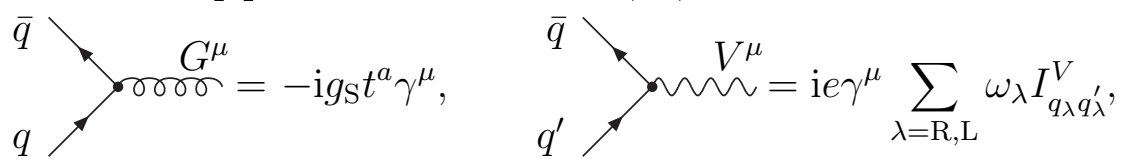

where $\omega_{\lambda}$ are the chiral projectors

$$
\omega_{\mathrm{R}}=\frac{1}{2}\left(1+\gamma_{5}\right), \quad \omega_{\mathrm{L}}=\frac{1}{2}\left(1-\gamma_{5}\right),
$$

$t^{a}$ are the Gell-Mann matrices and $I^{V}$ are matrices in the weak isospin space. For diagonal matrices such as $I^{A}$ and $I^{Z}$ we write $I_{q_{\lambda} q_{\lambda}^{\prime}}^{V}=\delta_{q q^{\prime}} I_{q_{\lambda}}^{V}$. In terms of the weak isospin $T_{q_{\lambda}}^{3}$ and the weak hypercharge $Y_{q_{\lambda}}$ we have

$$
I_{q_{\lambda}}^{Z}=\frac{c_{\mathrm{W}}}{s_{\mathrm{W}}} T_{q_{\lambda}}^{3}-\frac{s_{\mathrm{W}}}{c_{\mathrm{W}}} \frac{Y_{q_{\lambda}}}{2}, \quad I_{q_{\lambda}}^{A}=-Q_{q_{\lambda}}=-T_{q_{\lambda}}^{3}-\frac{Y_{q_{\lambda}}}{2},
$$

with the shorthands $c_{\mathrm{W}}=\cos \theta_{\mathrm{W}}$ and $s_{\mathrm{W}}=\sin \theta_{\mathrm{W}}$ for the weak mixing angle $\theta_{\mathrm{W}}$. The eigenvalues of isospin, hypercharge and $\mathrm{SU}(2)$ Casimir operators for left-handed fermions are

$$
T_{u_{\mathrm{L}}}^{3}=-T_{d_{\mathrm{L}}}^{3}=\frac{1}{2}, \quad Y_{u_{\mathrm{L}}}=Y_{d_{\mathrm{L}}}=\frac{1}{3}, \quad C_{\mathrm{F}}=\frac{3}{4}, \quad C_{\mathrm{A}}=2 .
$$

The only non-vanishing components of the generators associated with $W$ bosons are

$$
I_{u_{\mathrm{L}} d_{\mathrm{L}}}^{W^{+}}=I_{d_{\mathrm{L}} u_{\mathrm{L}}}^{W^{-}}=\frac{1}{\sqrt{2} s_{\mathrm{W}}}
$$

The triple gauge-bosons vertices read

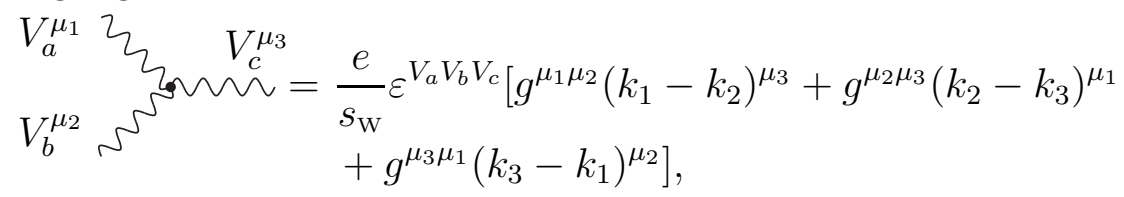

where the totally anti-symmetric tensor $\varepsilon^{V_{1} V_{2} V_{3}}$ is defined through the commutation relations

$$
\left[I^{V_{1}}, I^{V_{2}}\right]=\frac{\mathrm{i}}{s_{\mathrm{W}}} \sum_{V_{3}=A, Z, W^{ \pm}} \varepsilon^{V_{1} V_{2} V_{3}} I^{\bar{V}_{3}},
$$

and has components $\varepsilon^{Z W^{+} W^{-}}=-\mathrm{i} c_{\mathrm{W}}$ and $\varepsilon^{A W^{+} W^{-}}=\mathrm{i} s_{\mathrm{W}}$.

To lowest order in $\alpha$ and $\alpha_{\mathrm{S}}$, the unpolarized squared matrix element for the $\bar{q} q^{\prime} \rightarrow W^{\sigma} g$ process reads

$$
\bar{\sum}\left|\mathcal{M}_{0}^{\bar{q} q^{\prime} \rightarrow W^{\sigma} g}\right|^{2}=8 \pi^{2} \alpha \alpha_{\mathrm{S}}\left(N_{\mathrm{c}}^{2}-1\right)\left(I_{q_{\mathrm{L}} q_{\mathrm{L}}^{\prime}}^{W^{-\sigma}}\right)^{2} \frac{\hat{t}^{2}+\hat{u}^{2}+2 M_{W}^{2} \hat{s}}{\hat{t} \hat{u}}
$$

where $\alpha=e^{2} /(4 \pi)$ and $\alpha_{\mathrm{S}}=g_{\mathrm{S}}^{2} /(4 \pi)$ are the electromagnetic and the strong coupling constants. 


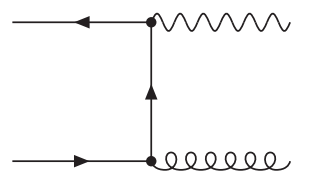

$(\mathrm{t} 1)$

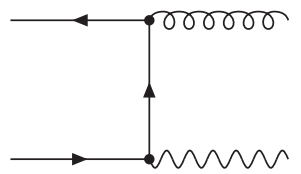

$(\mathrm{t} 2)$

Figure 1: Tree-level Feynman diagrams for the process $\bar{q} q^{\prime} \rightarrow W^{\sigma} g$.

\section{Virtual corrections}

In this section we present the virtual electroweak corrections to the $\bar{q} q^{\prime} \rightarrow W^{\sigma} g$ process. The algebraic reduction to gauge-coupling structures, standard matrix elements and one-loop scalar integrals is described in Sect. 3.2. The renormalization of ultraviolet divergences and the subtraction of infrared singularities originating from soft and collinear virtual photons are discussed in Sect. 3.3. and Sect. 3.4, respectively. In Sect. 3.5 we summarize the one-loop result for the unpolarized squared matrix element.

\subsection{Preliminaries}

As discussed in the previous section, the twelve different processes relevant for $W j$ production are related by $\mathrm{CP}$ and crossing symmetries. It is thus sufficient to consider only one of these processes. In the following we derive the one-loop corrections for the $\bar{q} q^{\prime} \rightarrow W^{\sigma} g$ process. The matrix element

$$
\mathcal{M}_{1}^{\bar{q} q^{\prime} \rightarrow W^{\sigma} g}=\mathcal{M}_{0}^{\bar{q} q^{\prime} \rightarrow W^{\sigma} g}+\delta \mathcal{M}_{1}^{\bar{q} q^{\prime} \rightarrow W^{\sigma} g}
$$

is expressed as a function of the Mandelstam invariants

$$
\hat{s}=\left(p_{\bar{q}}+p_{q^{\prime}}\right)^{2}, \quad \hat{t}=\left(p_{\bar{q}}-p_{W}\right)^{2}, \quad \hat{u}=\left(p_{q^{\prime}}-p_{W}\right)^{2} .
$$

The Born contribution $\mathcal{M}_{0}^{\bar{q} q^{\prime} \rightarrow W^{\sigma} g}$ results from the $t$ - and $u$-channel diagrams of Fig. 1. The loop and counterterm diagrams contributing to the corrections,

$$
\delta \mathcal{M}_{1}^{\bar{q} q^{\prime} \rightarrow W^{\sigma} g}=\delta \mathcal{M}_{1, \text { loops }}^{\bar{q} q^{\prime} \rightarrow W^{\sigma} g}+\delta \mathcal{M}_{1, \mathrm{CT}}^{\bar{q} q^{\prime} \rightarrow W^{\sigma} g},
$$

are depicted in Fig. 2 and Fig. 3, respectively.

The quarks that are present in the loop diagrams of Fig. 2 are treated as massless, and the regularization of the collinear singularities that arise in this limit is discussed in Sect. 3.4. The only quark-mass effects that we take into account are the $m_{t}$-terms that enter the counterterms through gauge-boson self-energies.

Our calculation has been performed at the matrix-element level and provides full control over polarization effects. However, at this level, the analytical expressions are too large to be published. Explicit results will thus be presented only for the unpolarized squared matrix element. 


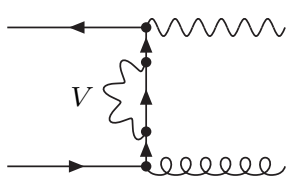

(s1)

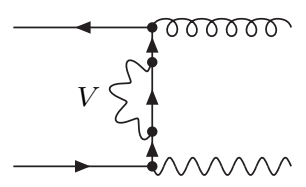

(s2)

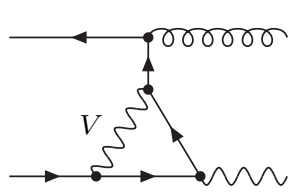

( v4)

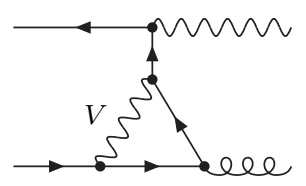

(v1)

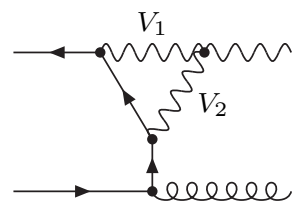

(v5)

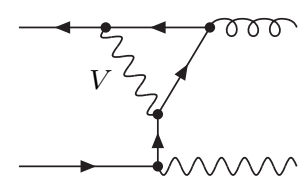

(v2)

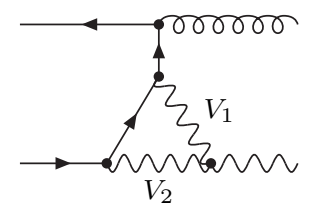

(v6)

(v3)

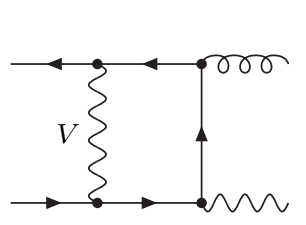

(b2)

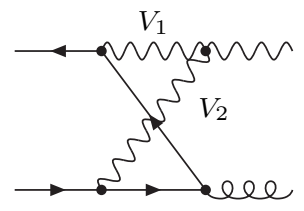

(b3)

Figure 2: One-loop Feynman diagrams for the process $\bar{q} q^{\prime} \rightarrow W^{\sigma} g$. The diagrams s1, s2, v1 and v2 receive contributions from neutral and charged gauge bosons, $V=A, Z, W^{ \pm}$. The diagrams $\mathrm{v} 3, \mathrm{v} 4, \mathrm{~b} 1$ and $\mathrm{b} 2$ involve only neutral gauge bosons, $V=A, Z$. The remaining diagrams, $\mathrm{v} 5, \mathrm{v} 6$ and $\mathrm{b} 3$ involve two contributions with one charged and one neutral gauge boson: $\left(V_{1}, V_{2}\right)=\left(V, W^{ \pm}\right)$and $\left(W^{ \pm}, V\right)$ with $V=A, Z$.

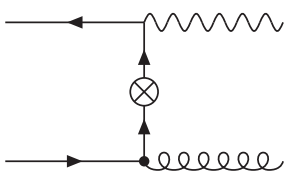

(c1)

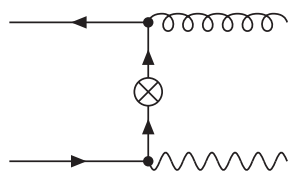

(c2)

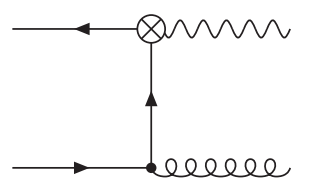

(c5)

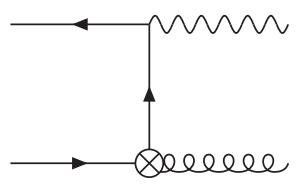

(c3)

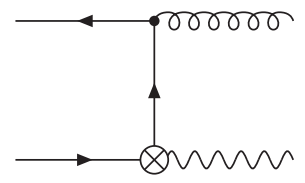

(c6)

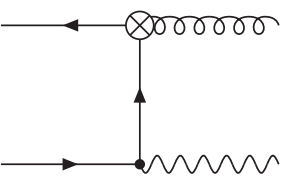

(c4)

Figure 3: Counterterm diagrams for the process $\bar{q} q^{\prime} \rightarrow W^{\sigma} g$. 


\subsection{Algebraic reduction}

The matrix element (33) has the general form

$$
\mathcal{M}_{1}^{\bar{q} q^{\prime} \rightarrow W^{\sigma} g}=\mathrm{i} e g_{\mathrm{S}} t^{a} \bar{v}\left(p_{\bar{q}}\right) \mathcal{M}_{1}^{\mathrm{L}, \mu \nu} \omega_{\mathrm{L}} u\left(p_{q^{\prime}}\right) \varepsilon_{\mu}^{*}\left(p_{W}\right) \varepsilon_{\nu}^{*}\left(p_{g}\right) .
$$

Since we neglect quark masses, $\mathcal{M}_{1}^{\mathrm{L}, \mu \nu}$ consists of terms involving an odd number of matrices $\gamma^{\rho}$ with $\rho=0, \ldots, 3$. The $\gamma^{5}$-terms are isolated in the chiral projector $\omega_{\mathrm{L}}$ defined in (26). The polarization dependence of the quark spinors and gauge-boson polarization vectors is implicitly understood. In analogy to (33) and (35) we write

$$
\mathcal{M}_{1}^{\mathrm{L}, \mu \nu}=\mathcal{M}_{0}^{\mathrm{L}, \mu \nu}+\delta \mathcal{M}_{1}^{\mathrm{L}, \mu \nu}, \quad \delta \mathcal{M}_{1}^{\mathrm{L}, \mu \nu}=\delta \mathcal{M}_{1, \text { loops }}^{\mathrm{L}, \mu \nu}+\delta \mathcal{M}_{1, \mathrm{C} T}^{\mathrm{L}, \mu \nu} .
$$

Following the approach adopted in Ref. [9], we isolate the $\mathrm{SU}(2) \times \mathrm{U}(1)$ couplings that appear in the Feynman diagrams and reduce the one-loop amplitude to a sum of contributions associated with independent coupling structures. As we will see, besides an abelian and a non-abelian contribution that are related to the ones found for $Z j$ production [9], for $W j$ production we have two additional coupling structures.

The coupling structure of the Born amplitude is trivial and consists simply of the $q_{\mathrm{L}} q_{\mathrm{L}}^{\prime}$ component of the $\mathrm{SU}(2)$ generator,

$$
\mathcal{M}_{0}^{\mathrm{L}, \mu \nu}=I_{q_{\mathrm{L}} q_{\mathrm{L}}^{\prime}}^{W^{-\sigma}} \mathcal{S}_{0}^{\mu \nu}=\frac{\mathcal{S}_{0}^{\mu \nu}}{\sqrt{2} s_{\mathrm{W}}}, \quad \mathcal{S}_{0}^{\mu \nu}=\frac{\gamma^{\mu}\left(\not p_{W}-\not p_{\bar{q}}\right) \gamma^{\nu}}{\hat{t}}+\frac{\gamma^{\nu}\left(\not p_{g}-\not \phi_{\bar{q}}\right) \gamma^{\mu}}{\hat{u}}
$$

The contribution of the loop diagrams of Fig. 2 can be written as

$$
\begin{aligned}
\delta \mathcal{M}_{1, \text { loops }}^{\mathrm{L}, \mu \nu}= & \frac{\alpha}{4 \pi}\left\{\sum_{V=\mathrm{A}, \mathrm{Z}, \mathrm{W}^{ \pm}}\left[\left(I^{W^{-\sigma}} I^{V} I^{\bar{V}}\right)_{q_{\mathrm{L}} q_{\mathrm{L}}^{\prime}} D_{1}^{\mu \nu}\left(M_{V}^{2}\right)+\left(I^{V} I^{\bar{V}} I^{W^{-\sigma}}\right)_{q_{\mathrm{L}} q_{\mathrm{L}}^{\prime}} D_{2}^{\mu \nu}\left(M_{V}^{2}\right)\right]\right. \\
& +\sum_{V=\mathrm{A}, \mathrm{Z}}\left[\left(I^{V} I^{W^{-\sigma}} I^{V}\right)_{q_{\mathrm{L}} q_{\mathrm{L}}^{\prime}} D_{3}^{\mu \nu}\left(M_{V}^{2}\right)+\frac{\mathrm{i}}{s_{\mathrm{W}}} \varepsilon^{W^{\sigma} V W^{-\sigma}}\left(I^{V} I^{W^{-\sigma}}\right)_{q_{\mathrm{L}} q_{\mathrm{L}}^{\prime}}\right. \\
& \left.\left.\times D_{4}^{\mu \nu}\left(M_{V}^{2}, M_{W}^{2}\right)+\frac{\mathrm{i}}{s_{\mathrm{W}}} \varepsilon^{V W^{\sigma} W^{-\sigma}}\left(I^{W^{-\sigma}} I^{V}\right)_{q_{\mathrm{L}} q_{\mathrm{L}}^{\prime}} D_{4}^{\mu \nu}\left(M_{W}^{2}, M_{V}^{2}\right)\right]\right\} .
\end{aligned}
$$

In the following, treating the electroweak gauge couplings as isospin matrices and using group-theoretical identities (see App. B of Ref. [23]), we express the above amplitude in terms of the eigenvalues of isospin, hypercharge and SU(2) Casimir operators for left-handed fermions (28).

The tensors $D_{1}^{\mu \nu}\left(M_{V}^{2}\right)$ and $D_{2}^{\mu \nu}\left(M_{V}^{2}\right)$ in (39) describe the contributions of the diagrams s1, v1 and s2, v2, respectively. These diagrams may involve charged or neutral virtual bosons. In the former case $\left(V=W^{ \pm}\right)$, the corresponding couplings read

$$
\sum_{\rho= \pm} I^{W^{-\sigma}} I^{W^{\rho}} I^{W^{-\rho}}=\sum_{\rho= \pm} I^{W^{\rho}} I^{W^{-\rho}} I^{W^{-\sigma}}=\frac{C_{\mathrm{F}}-\left(T^{3}\right)^{2}}{s_{\mathrm{W}}^{2}} I^{W^{-\sigma}} .
$$

\footnotetext{
${ }^{1}$ The following identities have to be understood as matrix identities, where the $q_{\mathrm{L}} q_{\mathrm{L}}^{\prime}$ indices of the $\mathrm{SU}(2)$ generators are implicitly understood.
} 
In the latter case $(V=A, Z)$ the coupling factors read

$$
\begin{aligned}
& I^{W^{-\sigma}} I^{V} I^{V}=\left[\delta_{V V}^{\mathrm{SU}(2)} \frac{\left(T^{3}\right)^{2}}{s_{\mathrm{w}}^{2}}+X_{V} T^{3} Y+\delta_{V V}^{\mathrm{U}(1)} \frac{Y^{2}}{4 c_{\mathrm{W}}^{2}}\right] I^{W^{-\sigma}}, \\
& I^{V} I^{V} I^{W^{-\sigma}}=\left[\delta_{V V}^{\mathrm{SU}(2)} \frac{\left(T^{3}\right)^{2}}{s_{\mathrm{W}}^{2}}-X_{V} T^{3} Y+\delta_{V V}^{\mathrm{U}(1)} \frac{Y^{2}}{4 c_{\mathrm{W}}^{2}}\right] I^{W^{-\sigma}} .
\end{aligned}
$$

Here

$$
\delta_{V V}^{\mathrm{SU}(2)}=\left(U_{V W^{3}}\right)^{2}, \quad X_{V}=\frac{U_{V W^{3}} U_{V B}}{s_{\mathrm{W}} c_{\mathrm{W}}}, \quad \delta_{V V}^{\mathrm{U}(1)}=\left(U_{V B}\right)^{2},
$$

where $U$ is the electroweak mixing matrix. For $V=A, Z$ we have $\delta_{A A}^{\mathrm{SU}(2)}=s_{\mathrm{w}}^{2}$, $X_{A}=-1, \delta_{A A}^{\mathrm{U}(1)}=c_{\mathrm{W}}^{2}$, and $\delta_{Z Z}^{\mathrm{SU}(2)}=c_{\mathrm{W}}^{2}, X_{Z}=1, \delta_{Z Z}^{\mathrm{U}(1)}=s_{\mathrm{w}}^{2}$. We note that

$$
\sum_{V=A, Z} \delta_{V V}^{\mathrm{SU}(2)}=\sum_{V=A, Z} \delta_{V V}^{\mathrm{U}(1)}=1, \quad \sum_{V=A, Z} X_{V}=0 .
$$

The tensor $D_{3}^{\mu \nu}\left(M_{V}^{2}\right)$ in (39) corresponds to the diagrams v3, v4, b1 and b2. These diagrams receive contributions from neutral virtual gauge bosons only $(V=A, Z)$. For the corresponding couplings we have

$$
I^{V} I^{W^{-\sigma}} I^{V}=\left[\delta_{V V}^{\mathrm{SU}(2)} \frac{C_{\mathrm{F}}-C_{\mathrm{A}} / 2}{s_{\mathrm{w}}^{2}}+\delta_{V V}^{\mathrm{U}(1)} \frac{Y^{2}}{4 c_{\mathrm{W}}^{2}}\right] I^{W^{-\sigma}} .
$$

Finally, $D_{4}^{\mu \nu}\left(M_{V_{1}}^{2}, M_{V_{2}}^{2}\right)$ represents the diagrams v5, v6 and b3. These diagrams involve a neutral gauge boson $(V=A, Z)$ and a $W$ boson. The coupling factors yield

$$
\begin{aligned}
& \frac{\mathrm{i}}{s_{\mathrm{W}}} \varepsilon^{W^{\sigma} V W^{-\sigma}} I^{V} I^{W^{-\sigma}}=\left[\delta_{V V}^{\mathrm{SU}(2)} \frac{C_{\mathrm{A}}}{4 s_{\mathrm{W}}^{2}}-X_{V} T^{3} Y\right] I^{W^{-\sigma}}, \\
& \frac{\mathrm{i}}{s_{\mathrm{W}}} \varepsilon^{V W^{\sigma} W^{-\sigma}} I^{W^{-\sigma}} I^{V}=\left[\delta_{V V}^{\mathrm{SU}(2)} \frac{C_{\mathrm{A}}}{4 s_{\mathrm{W}}^{2}}+X_{V} T^{3} Y\right] I^{W^{-\sigma}} .
\end{aligned}
$$

Using the above identities we express the one-loop amplitude (39) for $W$-boson production in a form that is analogous to the one adopted in Refs. [9, 10] to describe the production of neutral gauge bosons. To this end we define 2

$$
\begin{aligned}
\delta \mathcal{A}_{1, \mathrm{~A}}^{\mu \nu}\left(M_{V}^{2}\right) & =D_{1}^{\mu \nu}\left(M_{V}^{2}\right)+D_{2}^{\mu \nu}\left(M_{V}^{2}\right)+D_{3}^{\mu \nu}\left(M_{V}^{2}\right), \\
\delta \mathcal{A}_{1, \mathrm{~N}}^{\mu \nu}\left(M_{V}^{2}\right) & =\frac{1}{2}\left[D_{4}^{\mu \nu}\left(M_{V}^{2}, M_{W}^{2}\right)+D_{4}^{\mu \nu}\left(M_{W}^{2}, M_{V}^{2}\right)\right]-D_{3}^{\mu \nu}\left(M_{V}^{2}\right), \\
\delta \mathcal{A}_{1, \mathrm{X}}^{\mu \nu}\left(M_{V}^{2}\right) & =D_{1}^{\mu \nu}\left(M_{V}^{2}\right)+D_{2}^{\mu \nu}\left(M_{V}^{2}\right), \\
\delta \mathcal{A}_{1, \mathrm{Y}}^{\mu \nu}\left(M_{V}^{2}\right) & =D_{4}^{\mu \nu}\left(M_{V}^{2}, M_{W}^{2}\right)-D_{4}^{\mu \nu}\left(M_{W}^{2}, M_{V}^{2}\right)+D_{2}^{\mu \nu}\left(M_{V}^{2}\right)-D_{1}^{\mu \nu}\left(M_{V}^{2}\right) .
\end{aligned}
$$

\footnotetext{
${ }^{2}$ In our notation we emphasize the dependence of the form factors $\delta \mathcal{A}_{1, \mathrm{I}}^{\mu \nu}$ on $M_{V}$, whereas the dependence on the external momenta as well as the $M_{W}$-dependence (for $\delta \mathcal{A}_{1, \mathrm{~N}}^{\mu \nu}$ and $\delta \mathcal{A}_{1, \mathrm{Y}}^{\mu \nu}$ ) is implicitly understood.
} 
The tensor $\delta \mathcal{A}_{1, \mathrm{~A}}^{\mu \nu}\left(M_{V}^{2}\right)$ is identical to the abelian tensor defined in Ref. [9], and $\delta \mathcal{A}_{1, \mathrm{~N}}^{\mu \nu}\left(M_{V}^{2}\right)$ is equal to the non-abelian tensor of Ref. [9] for $M_{V}^{2}=M_{W}^{2}$. The remaining two tensors, $\delta \mathcal{A}_{1, \mathrm{X}}^{\mu \nu}\left(M_{V}^{2}\right)$ and $\delta \mathcal{A}_{1, \mathrm{Y}}^{\mu \nu}\left(M_{V}^{2}\right)$, are new. Using (40)-(46) we can write the one-loop amplitude (39) as

$$
\begin{aligned}
\delta \mathcal{M}_{1, \text { loops }}^{\mathrm{L}, \mu \nu}= & \frac{\alpha}{4 \pi \sqrt{2} s_{\mathrm{W}}}\left\{\sum _ { V = A , Z } \left[\left(\delta_{V V}^{\mathrm{SU}(2)} \frac{C_{\mathrm{F}}}{s_{\mathrm{W}}^{2}}+\delta_{V V}^{\mathrm{U}(1)} \frac{Y_{q_{\mathrm{L}}}^{2}}{4 c_{\mathrm{W}}^{2}}\right) \delta \mathcal{A}_{1, \mathrm{~A}}^{\mu \nu}\left(M_{V}^{2}\right)\right.\right. \\
& -\delta_{V V}^{\mathrm{SU}(2)} \frac{C_{\mathrm{F}}-\left(T_{q_{\mathrm{L}}}^{3}\right)^{2}}{s_{\mathrm{W}}^{2}} \delta \mathcal{A}_{1, \mathrm{X}}^{\mu \nu}\left(M_{V}^{2}\right)+\delta_{V V}^{\mathrm{SU}(2)} \frac{C_{\mathrm{A}}}{2 s_{\mathrm{W}}^{2}} \delta \mathcal{A}_{1, \mathrm{~N}}^{\mu \nu}\left(M_{V}^{2}\right) \\
& \left.\left.-X_{V} T_{q_{\mathrm{L}}}^{3} Y_{q_{\mathrm{L}}} \delta \mathcal{A}_{1, \mathrm{Y}}^{\mu \nu}\left(M_{V}^{2}\right)\right]+\frac{C_{\mathrm{F}}-\left(T_{q_{\mathrm{L}}}^{3}\right)^{2}}{s_{\mathrm{W}}^{2}} \delta \mathcal{A}_{1, \mathrm{X}}^{\mu \nu}\left(M_{W}^{2}\right)\right\}
\end{aligned}
$$

This amplitude has been reduced algebraically using the Dirac equation, the identity $p^{\mu} \varepsilon_{\mu}(p)=0$ for gauge-boson polarization vectors and Dirac algebra. Moreover, tensor loop integrals have been reduced to scalar ones by means of the PassarinoVeltman technique [24]. The result has been expressed in the form

$$
\delta \mathcal{A}_{1, \mathrm{I}}^{\mu \nu}\left(M_{V}^{2}\right)=\sum_{i=1}^{10} \sum_{j} \mathcal{F}_{\mathrm{I}}^{i j}\left(M_{V}^{2}\right) \mathcal{S}_{i}^{\mu \nu} J_{j}\left(M_{V}^{2}\right)
$$

for I=A,N,X,Y. The quantities $\mathcal{F}_{\mathrm{I}}^{i j}\left(M_{V}^{2}\right)$ are rational functions of Mandelstam invariants and masses. Explicit expressions for the tensors $\mathcal{S}_{i}^{\mu \nu}$ and the scalar loop integrals $J_{j}\left(M_{V}^{2}\right)$ are provided in Appendix B and Appendix $\mathrm{C}$.

\subsection{Renormalization}

While the tensors $\delta \mathcal{A}_{1, \mathrm{X}}^{\mu \nu}$ and $\delta \mathcal{A}_{1, \mathrm{Y}}^{\mu \nu}$ are ultraviolet finite, the abelian and the nonabelian tensors give rise to the ultraviolet singularities

$$
\left.\delta \mathcal{A}_{1, \mathrm{~A}}^{\mu \nu}\left(M_{V}^{2}\right)\right|_{\mathrm{UV}}=\bar{\Delta}_{\mathrm{UV}} \mathcal{S}_{0}^{\mu \nu},\left.\quad \delta \mathcal{A}_{1, \mathrm{~N}}^{\mu \nu}\left(M_{V}^{2}\right)\right|_{\mathrm{UV}}=2 \bar{\Delta}_{\mathrm{UV}} \mathcal{S}_{0}^{\mu \nu}
$$

where $\mathcal{S}_{0}^{\mu \nu}$ is the tensor structure of the Born amplitude (38), and

$$
\bar{\Delta}_{\mathrm{UV}}=\left(\frac{4 \pi \mu^{2}}{M_{Z}^{2}}\right)^{\varepsilon} \frac{\Gamma(1+\varepsilon)}{\varepsilon}=\frac{1}{\varepsilon}-\gamma_{\mathrm{E}}+\ln (4 \pi)+\ln \left(\frac{\mu^{2}}{M_{Z}^{2}}\right)+\mathcal{O}(\varepsilon)
$$

in $D=4-2 \varepsilon$ dimensions. These singularities are cancelled by the counterterm diagrams depicted in Fig. 3 and the results are independent of the scale $\mu$ of dimensional regularization. The counterterms that are responsible for the contributions of diagrams c1, c2, c3 and c4 read

$$
\longleftarrow \otimes \longleftarrow=\mathrm{i} \not p \omega_{\mathrm{L}} \delta Z_{q_{\mathrm{L}}}
$$$$
\$ 0 w=-\mathrm{i} g_{\mathrm{S}} t^{a} \gamma^{\mu} \omega_{\mathrm{L}} \delta Z_{q_{\mathrm{L}}}
$$ 
Since there is no $\mathcal{O}(\alpha)$ contribution to the renormalization of the strong coupling constant $g_{\mathrm{S}}$, these counterterms depend only on the wave-function renormalization constants for left-handed quarks, $\delta Z_{q_{\mathrm{L}}}$. Their combined contribution to the $\bar{q} q^{\prime} \rightarrow W^{\sigma} g$ process, i.e. the sum of the diagrams $\mathrm{c} 1, \mathrm{c} 2, \mathrm{c} 3$ and $\mathrm{c} 4$, vanishes. The renormalization of the $\bar{q} q^{\prime} \rightarrow W^{\sigma} g$ process is thus provided by the diagrams c5 and c6, which originate from the $W \bar{q} q^{\prime}$ counterterm,

with

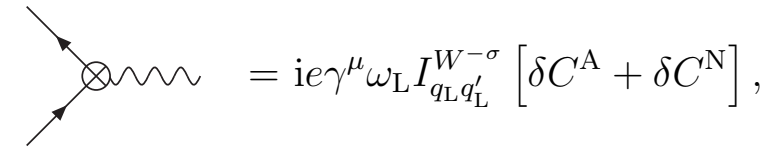

$$
\delta C^{\mathrm{A}}=\frac{1}{2}\left(\delta Z_{u_{\mathrm{L}}}+\delta Z_{d_{\mathrm{L}}}\right), \quad \delta C^{\mathrm{N}}=\frac{1}{2}\left(\delta Z_{W}+\frac{\delta g_{2}^{2}}{g_{2}^{2}}\right),
$$

and yields

$$
\delta \mathcal{M}_{1, \mathrm{CT}}^{\mathrm{L}, \mu \nu}=\left(\delta C^{\mathrm{A}}+\delta C^{\mathrm{N}}\right) \mathcal{M}_{0}^{\mathrm{L}, \mu \nu} .
$$

The wave-function renormalization constants of massless left-handed quarks and on-shell $W$ bosons are related to the corresponding self-energies by

$$
\delta Z_{q_{\mathrm{L}}}=-\operatorname{Re}\left(\Sigma^{q, \mathrm{~L}}(0)\right), \quad \delta Z_{W}=-\left.\operatorname{Re}\left(\frac{\partial \Sigma_{\mathrm{T}}^{W}\left(p^{2}\right)}{\partial p^{2}}\right)\right|_{p^{2}=M_{W}^{2}}
$$

and have been evaluated using the explicit results of Ref. [25].

For the definition and the renormalization of the $\mathrm{SU}(2)$ coupling constant,

$$
g_{2}^{2}=\frac{4 \pi \alpha}{s_{\mathrm{w}}^{2}}, \quad \frac{\delta g_{2}^{2}}{g_{2}^{2}}=\frac{\delta \alpha}{\alpha}-\frac{\delta s_{\mathrm{w}}^{2}}{s_{\mathrm{w}}^{2}},
$$

we adopt the $G_{\mu}$-scheme, where the electromagnetic coupling constant $\alpha$ is expressed in terms of the Fermi constant $G_{\mu}$, and the weak mixing angle is related to the onshell masses $M_{Z}, M_{W}$ of the gauge bosons,

$$
\alpha=\frac{\sqrt{2} G_{\mu} M_{W}^{2} s_{\mathrm{w}}^{2}}{\pi}, \quad s_{\mathrm{W}}^{2}=1-c_{\mathrm{W}}^{2}=1-M_{W}^{2} / M_{Z}^{2} .
$$

The counterterm $\delta \alpha / \alpha$ in the $G_{\mu}$-scheme can be derived from the on-shell counterterm $\delta \alpha(0) / \alpha(0)$ for the fine-structure constant in the Thompson limit. Using the one-loop relation $\alpha=\alpha(0)[1+\Delta r]$ and requiring $\alpha+\delta \alpha=\alpha(0)+\delta \alpha(0)$ we have

$$
\frac{\delta \alpha}{\alpha}=\frac{\delta \alpha(0)}{\alpha(0)}-\Delta r
$$

Combining the relations (56) -(58) and using the explicit one-loop expression for $\Delta r$ [26, 27], we obtain

$$
\frac{\delta g_{2}^{2}}{g_{2}^{2}}=\operatorname{Re}\left[\frac{\Sigma_{\mathrm{T}}^{W}\left(M_{W}^{2}\right)-\Sigma_{\mathrm{T}}^{W}(0)}{M_{W}^{2}}\right]-\frac{\alpha}{\pi s_{\mathrm{w}}^{2}}\left[\bar{\Delta}_{\mathrm{UV}}+\frac{1}{4}\left(6+\frac{7-12 s_{\mathrm{W}}^{2}}{2 s_{\mathrm{W}}^{2}} \ln \left(\frac{M_{W}^{2}}{M_{Z}^{2}}\right)\right)\right] .
$$


The above conterterms yield the ultraviolet singularities

$$
\begin{aligned}
\left.\delta C^{\mathrm{A}}\right|_{\mathrm{UV}} & =-\frac{\alpha}{8 \pi} \bar{\Delta}_{\mathrm{UV}} \sum_{q=u, d V=\mathrm{A}, \mathrm{Z}, \mathrm{W}^{ \pm}}\left(I^{V} I^{\bar{V}}\right)_{q_{\mathrm{L}}}=-\frac{\alpha}{4 \pi} \bar{\Delta}_{\mathrm{UV}}\left(\frac{C_{\mathrm{F}}}{s_{\mathrm{W}}^{2}}+\frac{Y_{q_{\mathrm{L}}^{2}}^{2}}{4 c_{\mathrm{W}}^{2}}\right), \\
\left.\delta C^{\mathrm{N}}\right|_{\mathrm{UV}} & =-\frac{\alpha}{2 \pi s_{\mathrm{W}}^{2}} \bar{\Delta}_{\mathrm{UV}}
\end{aligned}
$$

Using (43) one can easily verify that these singularities cancel those resulting from the loop diagrams [see (47) and (49)].

\subsection{Soft and collinear singularities}

Loop diagrams and wave-function renormalization constants involve singularities originating from soft and collinear virtual photons (for brevity denoted in the following as IR singularities). In order to isolate these singularities and check that they are cancelled by corresponding ones originating from real photon bremsstrahlung, we split the wave-function renormalization constants and the photon contributions to (47), i.e. the terms $\delta \mathcal{A}_{1, \mathrm{I}}^{\mu \nu}\left(M_{A}^{2}\right)$, in IR-singular (IR) and IR-finite (fin) parts:

$$
\begin{aligned}
\delta Z_{q_{\mathrm{L}}} & =\delta Z_{q_{\mathrm{L}}}^{\mathrm{IR}}+\delta Z_{q_{\mathrm{L}}}^{\mathrm{fin}}, \\
\delta Z_{W} & =\delta Z_{W}^{\mathrm{IR}}+\delta Z_{W}^{\mathrm{fin}} \\
\delta \mathcal{A}_{1, \mathrm{I}}^{\mu \nu}\left(M_{A}^{2}\right) & =\delta \mathcal{A}_{1, \mathrm{I}}^{\mathrm{IR}, \mu \nu}+\delta \mathcal{A}_{1, \mathrm{I}}^{\mathrm{fin}, \mu \nu} .
\end{aligned}
$$

The singular parts depend on the scheme adopted to regularize IR singularities. The remaining parts are scheme-independent and free from IR singularities, but can contain ultraviolet poles. For the regularization of IR singularities we use, alternatively, two different schemes:

- In the first scheme, which we denote as mass-regularization scheme (MR), we use infinitesimal quark masses $m$ and a photon-mass regulator, $M_{A}=\lambda$ with $0<\lambda \ll m$. Since the quark-mass dependence disappears in the final result, we perform the computation using the same mass $m$ for all quarks. To denote quantities evaluated in this scheme we use the label MR;

- In the second scheme we perform the calculation using massless fermions and photons, $M_{A}=m=0$, and we evaluate IR singularities in dimensional regularization (DR). To denote quantities evaluated in this scheme we use the label DR.

The singular parts of the wave-function renormalization constants read

$$
\begin{aligned}
& \delta Z_{q_{\mathrm{L}}}^{\mathrm{IR}}=\frac{\alpha}{4 \pi}\left(\frac{4 \pi \mu^{2}}{M_{W}^{2}}\right)^{\varepsilon} \Gamma(1+\varepsilon) Q_{q}^{2} h_{q}^{\mathrm{IR}}, \\
& \delta Z_{W}^{\mathrm{IR}}=\frac{\alpha}{4 \pi}\left(\frac{4 \pi \mu^{2}}{M_{W}^{2}}\right)^{\varepsilon} \Gamma(1+\varepsilon) h_{W}^{\mathrm{IR}}
\end{aligned}
$$


with

$$
h_{q, \mathrm{MR}}^{\mathrm{IR}}=-\ln \left(\frac{M_{W}^{2}}{m^{2}}\right)-2 \ln \left(\frac{\lambda^{2}}{m^{2}}\right)-4, \quad h_{W, \mathrm{MR}}^{\mathrm{IR}}=-2 \ln \left(\frac{\lambda^{2}}{M_{W}^{2}}\right),
$$

in the MR scheme and

$$
h_{q, \mathrm{DR}}^{\mathrm{IR}}=\frac{1}{\varepsilon}, \quad h_{W, \mathrm{DR}}^{\mathrm{IR}}=-\frac{2}{\varepsilon},
$$

in the DR scheme. The splitting of the loop contributions $\delta \mathcal{A}_{1, \mathrm{I}}^{\mu \nu}\left(M_{A}^{2}\right)$ into IR-singular and IR-finite parts is performed at the level of the scalar loop integrals $J_{i}\left(M_{A}^{2}\right)$ :

$$
J_{i}\left(M_{A}^{2}\right)=J_{i}^{\mathrm{IR}}+J_{i}^{\mathrm{fin}} .
$$

Explicit expression for the IR-singular and IR-finite parts of individual loop integrals are presented in Appendix D, Combining all singular contributions $J_{i}^{\mathrm{IR}}$ we obtain

$$
\delta \mathcal{A}_{1, \mathrm{I}}^{\mathrm{IR}, \mu \nu}=\left(\frac{4 \pi \mu^{2}}{M_{W}^{2}}\right)^{\varepsilon} \Gamma(1+\varepsilon) f_{\mathrm{I}}^{\mathrm{IR}} \mathcal{S}_{0}^{\mu \nu},
$$

i.e. the IR singularities factorize 3 with respect to the Born amplitude (38). The IR-singular part of the renormalized amplitude can be expressed in terms of the electromagnetic charges of the external particles as

$$
\delta \mathcal{M}_{1, \mathrm{IR}}^{\mathrm{L}, \mu \nu}=\frac{\alpha}{4 \pi}\left(\frac{4 \pi \mu^{2}}{M_{W}^{2}}\right)^{\varepsilon} \Gamma(1+\varepsilon)\left[-Q_{q} Q_{q^{\prime}} f_{1}^{\mathrm{IR}}+\sigma Q_{q} f_{2}^{\mathrm{IR}}-\sigma Q_{q^{\prime}} f_{3}^{\mathrm{IR}}\right] \mathcal{M}_{0}^{\mathrm{L}, \mu \nu},
$$

where $\sigma= \pm 1$ is the charge of the $W$ boson and

$$
\begin{aligned}
& f_{1}^{\mathrm{IR}}=-f_{\mathrm{A}}^{\mathrm{IR}}-h_{q}^{\mathrm{IR}}, \\
& f_{2}^{\mathrm{IR}}=-f_{\mathrm{A}}^{\mathrm{IR}}-f_{\mathrm{N}}^{\mathrm{IR}}+\frac{1}{2}\left(f_{\mathrm{X}}^{\mathrm{IR}}-f_{\mathrm{Y}}^{\mathrm{IR}}-h_{q}^{\mathrm{IR}}-h_{W}^{\mathrm{IR}}\right), \\
& f_{3}^{\mathrm{IR}}=-f_{\mathrm{A}}^{\mathrm{IR}}-f_{\mathrm{N}}^{\mathrm{IR}}+\frac{1}{2}\left(f_{\mathrm{X}}^{\mathrm{IR}}+f_{\mathrm{Y}}^{\mathrm{IR}}-h_{q}^{\mathrm{IR}}-h_{W}^{\mathrm{IR}}\right) .
\end{aligned}
$$

In the MR scheme we obtain

$$
\begin{aligned}
f_{1, \mathrm{MR}}^{\mathrm{IR}}= & -2 \ln \left(\frac{\lambda^{2}}{M_{W}^{2}}\right) \ln \left(\frac{-\hat{s}}{m^{2}}\right)-\ln ^{2}\left(\frac{m^{2}}{M_{W}^{2}}\right)+3 \ln \left(\frac{m^{2}}{M_{W}^{2}}\right)+2 \ln \left(\frac{\lambda^{2}}{m^{2}}\right), \\
f_{2, \mathrm{MR}}^{\mathrm{IR}}= & \ln \left(\frac{\lambda^{2}}{M_{W}^{2}}\right)\left[\ln \left(\frac{m^{2}}{M_{W}^{2}}\right)-2 \ln \left(1-\frac{\hat{t}}{M_{W}^{2}}\right)\right]-\frac{1}{2} \ln ^{2}\left(\frac{m^{2}}{M_{W}^{2}}\right) \\
& +\frac{1}{2} \ln \left(\frac{m^{2}}{M_{W}^{2}}\right)+2 \ln \left(\frac{\lambda^{2}}{M_{W}^{2}}\right), \\
f_{3, \mathrm{MR}}^{\mathrm{IR}}= & \left.f_{2, \mathrm{MR}}^{\mathrm{IR}}\right|_{\hat{t} \rightarrow \hat{u}}
\end{aligned}
$$

\footnotetext{
${ }^{3}$ To be precise, the tensors $\delta \mathcal{A}_{1, \mathrm{X}}^{\mu \nu}\left(M_{A}^{2}\right)$ and $\delta \mathcal{A}_{1, \mathrm{~N}}^{\mu \nu}\left(M_{A}^{2}\right)$ contain also non-factorizable IR divergences. However these non-factorizable singularities are related by

$$
\delta \mathcal{A}_{1, \mathrm{X}}^{\text {non }} \text { fact }, \mu \nu^{2}\left(M_{A}^{2}\right)=2 \delta \mathcal{A}_{1, \mathrm{~N}}^{\text {non-fact }, \mu \nu}\left(M_{A}^{2}\right),
$$

and due to the identity $C_{\mathrm{F}}-\left(T_{q_{\mathrm{L}}}^{3}\right)^{2}=C_{\mathrm{A}} / 4$, which relates the coupling structures associated with the $\mathrm{X}$ - and N-terms in (47), they cancel.
} 
and in the DR scheme

$$
\begin{aligned}
& f_{1, \mathrm{DR}}^{\mathrm{IR}}=\frac{2}{\varepsilon^{2}}-\frac{1}{\varepsilon}\left[2 \ln \left(\frac{-\hat{s}}{M_{W}^{2}}\right)-3\right]+4, \\
& f_{2, \mathrm{DR}}^{\mathrm{IR}}=\frac{1}{\varepsilon^{2}}-\frac{1}{\varepsilon}\left[2 \ln \left(1-\frac{\hat{t}}{M_{W}^{2}}\right)-\frac{5}{2}\right]+2, \\
& f_{3, \mathrm{DR}}^{\mathrm{IR}}=\left.f_{2, \mathrm{DR}}^{\mathrm{IR}}\right|_{\hat{t} \rightarrow \hat{u}} .
\end{aligned}
$$

The splitting (61) has been performed in such a way that in the high-energy limit $\left(\hat{s},|\hat{t}|,|\hat{u}| \gg M_{W}^{2}\right)$ the IR-finite part of the amplitude has the same logarithmic behaviour as the virtual corrections regularized by a photon mass $M_{A}=M_{W}$. Indeed the IR-singular parts $f_{i}^{\mathrm{IR}}$ correspond exactly to the contribution called purely electromagnetic in Ref. [14]. This implies that, up to terms that are not logarithmically enhanced at high energies, the IR-finite part of the corrections corresponds to the symmetric electroweak contribution of Ref. [14], which is constructed by setting the photon mass equal to $M_{W}$. This property is evident in the asymptotic high-energy expressions (80) for the IR-finite part of the diagrams involving virtual photons.

\subsection{Result}

Let us summarize our result for the unpolarized squared matrix element for the $\bar{q} q^{\prime} \rightarrow W^{\sigma} g$ process. To $\mathcal{O}\left(\alpha^{2} \alpha_{\mathrm{S}}\right)$,

$$
\bar{\sum}\left|\mathcal{M}_{1}^{\bar{q} q^{\prime} \rightarrow W^{\sigma} g}\right|^{2}=\bar{\sum}\left|\mathcal{M}_{0}^{\bar{q} q^{\prime} \rightarrow W^{\sigma} g}\right|^{2}+2 \operatorname{Re}\left[\bar{\sum}\left(\mathcal{M}_{0}^{\bar{q} q^{\prime} \rightarrow W^{\sigma} g}\right)^{*} \delta \mathcal{M}_{1}^{\bar{q} q^{\prime} \rightarrow W^{\sigma} g}\right] .
$$

Using (36) and summing over the polarizations we can express the interference term as

$$
\begin{aligned}
2 \operatorname{Re} & {\left[\bar{\sum}\left(\mathcal{M}_{0}^{\bar{q} q^{\prime} \rightarrow W^{\sigma} g}\right)^{*} \delta \mathcal{M}_{1}^{\bar{q} q^{\prime} \rightarrow W^{\sigma} g}\right]=2 \pi^{2} \alpha \alpha_{\mathrm{S}}\left(N_{c}^{2}-1\right) } \\
& \times \operatorname{Re}\left[\operatorname{Tr}\left(\not p_{q^{\prime}} \overline{\mathcal{M}}_{0}^{\mathrm{L}, \mu \nu} \not \phi_{\bar{q}} \delta \mathcal{M}_{1}^{\mathrm{L}, \mu^{\prime} \nu^{\prime}}\right)\right] g_{\nu \nu^{\prime}}\left(g_{\mu \mu^{\prime}}-\frac{p_{W \mu} p_{W \mu^{\prime}}}{p_{W}^{2}}\right),
\end{aligned}
$$

where $\overline{\mathcal{M}}=\gamma^{0} \mathcal{M}^{\dagger} \gamma^{0}$. Combining the contributions of the bare one-loop diagrams (47) and the counterterms (54) yields

$$
\begin{aligned}
\bar{\sum}\left|\mathcal{M}_{1}^{\bar{q} q^{\prime} \rightarrow W^{\sigma} g}\right|^{2}= & {\left[1+2 \operatorname{Re}\left(\delta C^{\mathrm{A}}+\delta C^{\mathrm{N}}\right)\right] \bar{\sum}\left|\mathcal{M}_{0}^{\bar{q} q^{\prime} \rightarrow W^{\sigma} g}\right|^{2}+\frac{2 \pi \alpha^{2} \alpha_{\mathrm{S}}}{s_{\mathrm{W}}^{2}}\left(N_{\mathrm{c}}^{2}-1\right) } \\
& \times \operatorname{Re}\left\{\sum _ { V = \mathrm { A } , \mathrm { Z } } \left[\left(\delta_{V V}^{\mathrm{SU}(2)} \frac{C_{\mathrm{F}}}{s_{\mathrm{W}}^{2}}+\delta_{V V}^{\mathrm{U}(1)} \frac{Y_{q_{\mathrm{L}}}^{2}}{4 c_{\mathrm{W}}^{2}}\right) H_{1}^{\mathrm{A}}\left(M_{V}^{2}\right)\right.\right. \\
& -\delta_{V V}^{\mathrm{SU}(2)} \frac{C_{\mathrm{F}}-\left(T_{q_{\mathrm{L}}}^{3}\right)^{2}}{s_{\mathrm{W}}^{2}} H_{1}^{\mathrm{X}}\left(M_{V}^{2}\right)+\delta_{V V}^{\mathrm{SU}(2)} \frac{C_{\mathrm{A}}}{2 s_{\mathrm{W}}^{2}} H_{1}^{\mathrm{N}}\left(M_{V}^{2}\right) \\
& \left.\left.-X_{V} T_{q_{\mathrm{L}}}^{3} Y_{q_{\mathrm{L}}} H_{1}^{\mathrm{Y}}\left(M_{V}^{2}\right)\right]+\frac{C_{\mathrm{F}}-\left(T_{q_{\mathrm{L}}}^{3}\right)^{2}}{s_{\mathrm{W}}^{2}} H_{1}^{\mathrm{X}}\left(M_{W}^{2}\right)\right\} .
\end{aligned}
$$


The unpolarized Born contribution is given in (32), the counterterms $\delta C^{\mathrm{A}}$ and $\delta C^{\mathrm{N}}$ are presented in Sect. 3.3, and the coupling factors are specified by (28) and (42). The functions $H_{1}^{\mathrm{I}}\left(M_{V}^{2}\right)$ represent the contributions resulting from the loop diagrams of Fig. 2. They are related to the tensors $\delta \mathcal{A}_{1, \mathrm{I}}^{\mu \nu}\left(M_{V}^{2}\right)$ in (47)-(48) by

$$
H_{1}^{\mathrm{I}}\left(M_{V}^{2}\right)=\frac{1}{8} \operatorname{Tr}\left[\not p_{q^{\prime}} \overline{\mathcal{S}}_{0}^{\mu \nu} \not p_{\bar{q}} \delta \mathcal{A}_{1, \mathrm{I}}^{\mu^{\prime} \nu^{\prime}}\left(M_{V}^{2}\right)\right] g_{\nu \nu^{\prime}}\left(g_{\mu \mu^{\prime}}-\frac{p_{W \mu} p_{W \mu^{\prime}}}{p_{W}^{2}}\right) .
$$

The couplings associated with $H_{1}^{\mathrm{A}}, H_{1}^{\mathrm{N}}$ and $H_{1}^{\mathrm{X}}$ are the same for $q=u$ and $q=$ $d$. Thus the crossing and CP symmetry relations (21) and (22) imply that these functions are symmetric with respect to the transformation $\hat{t} \leftrightarrow \hat{u}$. In contrast, $H_{1}^{\mathrm{Y}}$ is antisymmetric with respect to $\hat{t} \leftrightarrow \hat{u}$ exchange since the corresponding coupling is proportional to $T_{q_{\mathrm{L}}}^{3}$ and has thus opposite signs for $q=u$ and $q=d$. The functions $H_{1}^{\mathrm{I}}\left(M_{V}^{2}\right)$ are presented in Appendix $\mathrm{E}$ as linear combinations of scalar loop integrals. We note that, in contrast to the definition adopted in the case of $Z j$ production [9], here we do not include the contributions of the fermionic wavefunction renormalization constants in $H_{1}^{\mathrm{A}}\left(M_{V}^{2}\right)$.

For the IR-singular part of the renormalized one-loop correction we obtain

$$
\begin{aligned}
\bar{\sum}\left|\mathcal{M}_{1, \mathrm{IR}}^{\bar{q} q^{\prime} \rightarrow W^{\sigma} g}\right|^{2}= & \frac{\alpha}{2 \pi} \operatorname{Re}\left[-Q_{q} Q_{q^{\prime}} f_{1}^{\mathrm{IR}}+\sigma Q_{q} f_{2}^{\mathrm{IR}}-\sigma Q_{q^{\prime}} f_{3}^{\mathrm{IR}}\right] \\
& \times\left(\frac{4 \pi \mu^{2}}{M_{W}^{2}}\right)^{\varepsilon} \Gamma(1+\varepsilon) \bar{\sum}\left|\mathcal{M}_{0}^{\bar{q} q^{\prime} \rightarrow W^{\sigma} g}\right|^{2} .
\end{aligned}
$$

The IR-singular functions $f_{i}^{\mathrm{IR}}$ in the MR and DR schemes are presented in Sect. 3.4.

\section{High-energy limit}

In this section we provide compact analytic expressions that describe the behaviour of the IR-finite part of the virtual electroweak corrections in the limit $M_{W}^{2} / \hat{s} \rightarrow 0$ with $\hat{t} / \hat{s}$ and $\hat{u} / \hat{s}$ constant. In this limit, which is applicable for transverse momenta of $\mathcal{O}(100 \mathrm{GeV})$ or beyond, the electroweak corrections are dominated by logarithmic contributions of the type $\ln \left(\hat{s} / M_{W}^{2}\right)$. In Sect. 4.1 we present the asymptotic expansion of the one-loop corrections, including leading and next-to-leading logarithms, as well as terms that are not logarithmically enhanced at high energies. In Sect. 4.2 we present the two-loop corrections to next-to-leading logarithmic accuracy.

\subsection{Next-to-next-to-leading approximation at one loop}

In this section we discuss the high-energy behaviour of the IR-finite part of the one-loop corrections to the $\bar{q} q^{\prime} \rightarrow W^{\sigma} g$ process, obtained by subtracting the IR divergence (75) from the renormalized one-loop result (73),

$$
\bar{\sum}\left|\mathcal{M}_{1, \mathrm{fin}}^{\bar{q} q^{\prime} \rightarrow W^{\sigma} g}\right|^{2}=\bar{\sum}\left|\mathcal{M}_{1}^{\bar{q} q^{\prime} \rightarrow W^{\sigma} g}\right|^{2}-\bar{\sum}\left|\mathcal{M}_{1, \mathrm{IR}}^{\bar{q} q^{\prime} \rightarrow W^{\sigma} g}\right|^{2} .
$$


In the following we present explicit asymptotic expressions for the unrenormalized loop contributions, i.e. for the IR-finite parts $H_{1}^{\mathrm{I}, \mathrm{fin}}$ of the functions $H_{1}^{\mathrm{I}}$ in (73). Using the general results of Ref. [28], we evaluate the functions $H_{1}^{\mathrm{I}, \text { fin }}$ to next-tonext-to-leading logarithmic (NNLL) accuracy. This approximation accounts for all contributions that are not suppressed by powers of $M_{W}^{2} / \hat{s}$. It includes double and single logarithms as well as terms that are not logarithmically enhanced in the highenergy limit. To simplify non-logarithmic functions of the ratio $M_{Z} / M_{W}$ we have performed an expansion in $s_{\mathrm{w}}^{2}=1-M_{W}^{2} / M_{Z}^{2}$, keeping only terms up to the first order 4 in $s_{\mathrm{W}}^{2}$. The NNLL expansion of $H_{1}^{\mathrm{I}, \mathrm{fin}}\left(M_{V}^{2}\right)$ has the general form

$$
H_{1}^{\mathrm{I}, \mathrm{fin}}\left(M_{V}^{2}\right) \stackrel{\mathrm{NNLL}}{=} \operatorname{Re}\left[g_{0}^{\mathrm{I}}\left(M_{V}^{2}\right) \frac{\hat{t}^{2}+\hat{u}^{2}}{\hat{t} \hat{u}}+g_{1}^{\mathrm{I}}\left(M_{V}^{2}\right) \frac{\hat{t}^{2}-\hat{u}^{2}}{\hat{t} \hat{u}}+g_{2}^{\mathrm{I}}\left(M_{V}^{2}\right)\right] .
$$

It involves the rational function $\left(\hat{t}^{2}+\hat{u}^{2}\right) / \hat{t} \hat{u}$, which has the same angular behaviour as the squared Born amplitude (32) in the high-energy limit, and two other rational functions, which describe different angular dependencies. The functions $g_{i}^{\mathrm{I}}$ consist of logarithms of the kinematical variables and constants. The loop diagrams involving $Z$ and $W$ bosons, with mass $M_{V}=M_{Z}, M_{W}$, yield

$$
\begin{aligned}
g_{0}^{\mathrm{N}}\left(M_{V}^{2}\right)= & 2\left[\bar{\Delta}_{\mathrm{UV}}+\ln \left(\frac{M_{Z}^{2}}{M_{W}^{2}}\right)+\ln \left(\frac{M_{V}^{2}}{M_{W}^{2}}\right)\right]+\ln ^{2}\left(\frac{-\hat{s}}{M_{V}^{2}}\right)-\frac{1}{2}\left[\ln ^{2}\left(\frac{-\hat{t}}{M_{V}^{2}}\right)\right. \\
& \left.+\ln ^{2}\left(\frac{-\hat{t}}{M_{W}^{2}}\right)+\ln ^{2}\left(\frac{-\hat{u}}{M_{V}^{2}}\right)+\ln ^{2}\left(\frac{-\hat{u}}{M_{W}^{2}}\right)\right]+\ln ^{2}\left(\frac{\hat{t}}{\hat{u}}\right)-\frac{3}{2}\left[\ln ^{2}\left(\frac{\hat{t}}{\hat{s}}\right)\right. \\
& \left.+\ln ^{2}\left(\frac{\hat{u}}{\hat{s}}\right)\right]-\frac{20 \pi^{2}}{9}-\frac{2 \pi}{\sqrt{3}}+4, \\
g_{1}^{\mathrm{N}}\left(M_{V}^{2}\right)= & \frac{1}{2}\left[\ln ^{2}\left(\frac{\hat{u}}{\hat{s}}\right)-\ln ^{2}\left(\frac{\hat{t}}{\hat{s}}\right)\right], \\
g_{2}^{\mathrm{N}}\left(M_{V}^{2}\right)= & -2\left[\ln ^{2}\left(\frac{\hat{t}}{\hat{s}}\right)+\ln ^{2}\left(\frac{\hat{u}}{\hat{s}}\right)+\ln \left(\frac{\hat{t}}{\hat{s}}\right)+\ln \left(\frac{\hat{u}}{\hat{s}}\right)\right]+2 \ln \left(\frac{M_{V}^{2}}{M_{W}^{2}}\right)-4 \pi^{2}, \\
g_{0}^{\mathrm{A}}\left(M_{V}^{2}\right)= & -\ln ^{2}\left(\frac{-\hat{s}}{M_{V}^{2}}\right)+3 \ln \left(\frac{-\hat{s}}{M_{V}^{2}}\right)+\frac{3}{2}\left[\ln ^{2}\left(\frac{\hat{t}}{\hat{s}}\right)+\ln ^{2}\left(\frac{\hat{u}}{\hat{s}}\right)+\ln ^{2}\left(\frac{\hat{t}}{\hat{s}}\right)\right. \\
& \left.+\ln ^{2}\left(\frac{\hat{u}}{\hat{s}}\right)\right]+\frac{7 \pi^{2}}{3}-\frac{5}{2}+g_{0}^{\mathrm{A}, \mathrm{UV}}\left(M_{V}^{2}\right), \\
g_{1}^{\mathrm{A}}\left(M_{V}^{2}\right)= & -g_{1}^{\mathrm{N}}\left(M_{W}^{2}\right)+\frac{3}{2}\left[\ln \left(\frac{\hat{u}}{\hat{s}}\right)-\ln \left(\frac{\hat{t}}{\hat{s}}\right)\right], \\
g_{2}^{\mathrm{A}}\left(M_{V}^{2}\right)= & -g_{2}^{\mathrm{N}}\left(M_{W}^{2}\right), \\
g_{0}^{\mathrm{X}}\left(M_{V}^{2}\right)= & 0, \\
g_{1}^{\mathrm{X}}\left(M_{V}^{2}\right)= & 0,
\end{aligned}
$$

\footnotetext{
${ }^{4}$ In practice we find that all terms of $\mathcal{O}\left(s_{\mathrm{w}}^{2}\right)$ cancel in the result.
} 


$$
\begin{aligned}
& g_{2}^{\mathrm{X}}\left(M_{V}^{2}\right)=-2\left[2 \ln \left(\frac{-\hat{s}}{M_{V}^{2}}\right)+\ln \left(\frac{\hat{t}}{\hat{s}}\right)+\ln \left(\frac{\hat{u}}{\hat{s}}\right)-3\right], \\
& g_{0}^{\mathrm{Y}}\left(M_{V}^{2}\right)=\ln ^{2}\left(\frac{-\hat{t}}{M_{W}^{2}}\right)-\ln ^{2}\left(\frac{-\hat{t}}{M_{V}^{2}}\right)-\ln ^{2}\left(\frac{-\hat{u}}{M_{W}^{2}}\right)+\ln ^{2}\left(\frac{-\hat{u}}{M_{V}^{2}}\right), \\
& g_{1}^{\mathrm{Y}}\left(M_{V}^{2}\right)=0 \\
& g_{2}^{\mathrm{Y}}\left(M_{V}^{2}\right)=2 \ln \left(\frac{\hat{t}}{\hat{u}}\right),
\end{aligned}
$$

where $\bar{\Delta}_{\mathrm{UV}}$ is defined in (50) and, in order to facilitate the comparison with Ref. [9], we have isolated the term

$$
g_{0}^{\mathrm{A}, \mathrm{UV}}\left(M_{V}^{2}\right)=\bar{\Delta}_{\mathrm{UV}}+\ln \left(\frac{M_{Z}^{2}}{M_{V}^{2}}\right)-\frac{1}{2} .
$$

If we included the fermionic wave-function renormalization constants in the definition of the function $H_{1}^{\mathrm{A}}$, as we had done for the case of $Z j$ production in Ref. 9], this term would cancel and the function $g_{0}^{\mathrm{A}}\left(M_{V}^{2}\right)$ would be identical to the one obtained in Ref. [9].

For the loop diagrams involving photons $\left(M_{V}=M_{A}\right)$, after subtraction of the IR-singular parts, we obtain

$$
\begin{aligned}
g_{0}^{\mathrm{N}}\left(M_{A}^{2}\right) & =g_{0}^{\mathrm{N}}\left(M_{W}^{2}\right)-\frac{7 \pi^{2}}{9}+\frac{2 \pi}{\sqrt{3}}, \\
g_{0}^{\mathrm{A}}\left(M_{A}^{2}\right) & =g_{0}^{\mathrm{A}}\left(M_{W}^{2}\right)+\pi^{2}, \\
g_{0}^{\mathrm{I}}\left(M_{A}^{2}\right) & =g_{0}^{\mathrm{I}}\left(M_{W}^{2}\right) \quad \text { for } \quad \mathrm{I}=\mathrm{X}, \mathrm{Y}, \\
g_{1}^{\mathrm{I}}\left(M_{A}^{2}\right) & =g_{1}^{\mathrm{I}}\left(M_{W}^{2}\right) \quad \text { for } \quad \mathrm{I}=\mathrm{A}, \mathrm{N}, \mathrm{X}, \mathrm{Y}, \\
g_{2}^{\mathrm{I}}\left(M_{A}^{2}\right) & =g_{2}^{\mathrm{I}}\left(M_{W}^{2}\right) \quad \text { for } \quad \mathrm{I}=\mathrm{A}, \mathrm{N}, \mathrm{X}, \mathrm{Y} .
\end{aligned}
$$

The contribution of the counterterms $\delta C^{\mathrm{A}}$ and $\delta C^{\mathrm{N}}$ to the IR-finite part of the renormalized result (76) is obtained by subtracting from (53) the IR-divergent part of the wave-function renormalization constants (62). This contribution, consisting of on-shell self-energies and their derivatives, does not depend on the scattering energy. Therefore we evaluate the IR-finite parts of the counterterms in numerical form without applying any approximation. Using the input parameters specified in Sect. 7 we obtain

$$
\begin{aligned}
& \delta C^{\mathrm{A}, \mathrm{fin}}=\frac{1}{2}\left(\delta Z_{u_{\mathrm{L}}}^{\mathrm{fin}}+\delta Z_{d_{\mathrm{L}}}^{\mathrm{fin}}\right)=\left.\delta C^{\mathrm{A}}\right|_{\mathrm{UV}}+5.57 \times 10^{-4}, \\
& \delta C^{\mathrm{N}, \mathrm{fin}}=\frac{1}{2}\left(\delta Z_{W}^{\mathrm{fin}}+\frac{\delta g_{2}^{2}}{g_{2}^{2}}\right)=\left.\delta C^{\mathrm{N}}\right|_{\mathrm{UV}}-1.49 \times 10^{-3} .
\end{aligned}
$$

The UV divergences $\left.\delta C^{\mathrm{A}, \mathrm{N}}\right|_{\mathrm{UV}}\left[\right.$ see (60)] cancel against the $\bar{\Delta}_{\mathrm{UV}}$-terms in (178)-(80) .

The results (78) - (80), for the $\bar{q} q^{\prime} \rightarrow W^{\sigma} g$ process, are valid for arbitrary values of the Mandelstam invariants and can easily be translated to all other processes in 
(2) by means of the relations (21)-(22). Logarithms with negative arguments in (78) -(80) are defined through the usual i $\varepsilon$ prescription, $\hat{r} \rightarrow \hat{r}+\mathrm{i} \varepsilon$ for $\hat{r}=\hat{s}, \hat{t}, \hat{u}$.

In next-to-leading logarithmic (NLL) approximation, i.e. retaining only double and single logarithms that grow with energy, the above results assume a particularly compact form. In this approximation the counterterms do not contribute,

$$
\delta C^{\mathrm{A}} \stackrel{\mathrm{NLL}}{=} \delta C^{\mathrm{N}} \stackrel{\mathrm{NLL}}{=} 0
$$

and for the functions $H_{1}^{\mathrm{I} \text {,fin }}\left(M_{V}^{2}\right)$, neglecting logarithms of $M_{Z} / M_{W}$, we obtain

$$
\begin{aligned}
& H_{1}^{\mathrm{N}, \mathrm{fin}}\left(M_{V}^{2}\right) \stackrel{\mathrm{NLL}}{=}-\left[\ln ^{2}\left(\frac{|\hat{t}|}{M_{W}^{2}}\right)+\ln ^{2}\left(\frac{|\hat{u}|}{M_{W}^{2}}\right)-\ln ^{2}\left(\frac{|\hat{s}|}{M_{W}^{2}}\right)\right] \frac{\hat{t}^{2}+\hat{u}^{2}}{\hat{t} \hat{u}}, \\
& H_{1}^{\mathrm{A}, \mathrm{fin}}\left(M_{V}^{2}\right) \stackrel{\mathrm{NLL}}{=}-\left[\ln ^{2}\left(\frac{|\hat{s}|}{M_{W}^{2}}\right)-3 \ln \left(\frac{|\hat{s}|}{M_{W}^{2}}\right)\right] \frac{\hat{t}^{2}+\hat{u}^{2}}{\hat{t} \hat{u}}, \\
& H_{1}^{\mathrm{X}, \mathrm{fin}}\left(M_{V}^{2}\right) \stackrel{\mathrm{NLL}}{=}-4 \ln \left(\frac{|\hat{s}|}{M_{W}^{2}}\right), \\
& H_{1}^{\mathrm{A}, \mathrm{fin}}\left(M_{V}^{2}\right) \stackrel{\mathrm{NLL}}{=} 0
\end{aligned}
$$

for $V=A, Z, W$. We note that, owing to $H_{1}^{\mathrm{X}, \mathrm{fin}}\left(M_{Z}\right) \stackrel{\mathrm{NLL}}{=} H_{1}^{\mathrm{X}, \mathrm{fin}}\left(M_{A}\right)$ and (43), the NLL contribution of the function $H_{1}^{\mathrm{X}, \mathrm{fin}}\left(M_{V}\right)$ cancels in (73). Thus the NLL corrections (83) are proportional to the rational function $\left(\hat{t}^{2}+\hat{u}^{2}\right) / \hat{t} \hat{u}$, which describes the angular dependence of the Born cross section.

\subsection{Next-to-leading logarithms up to two loops}

Let us now present our results for the NLL asymptotic behaviour of the electroweak corrections up to two loops. For a discussion of the calculation we refer to Ref. [8], where the same class of corrections has been computed for $Z j$ production. The results have been obtained in the $M_{Z}=M_{W}$ approximation. As in the previous section, we present results for the IR-finite part of the electroweak corrections, obtained after subtraction of IR singularities. As discussed in Sect. 3.4, at one loop this subtraction is performed in such a way that, to NLL accuracy, the IR-finite part corresponds to the complete electroweak correction regularized with a fictitious photon mass $M_{A}=M_{W}$. The same prescription is adopted at the two-loop level.

The unpolarized squared matrix element for $\bar{q} q^{\prime} \rightarrow W^{\sigma} g$, including NLL terms up to the two-loop level, has the general form

$$
\bar{\sum}\left|\mathcal{M}_{2}^{\bar{q} q^{\prime} \rightarrow W^{\sigma} g}\right|^{2}=8 \pi^{2} \alpha \alpha_{\mathrm{S}}\left(N_{\mathrm{c}}^{2}-1\right) \frac{\hat{t}^{2}+\hat{u}^{2}}{\hat{t} \hat{u}}\left[A^{(0)}+\left(\frac{\alpha}{2 \pi}\right) A^{(1)}+\left(\frac{\alpha}{2 \pi}\right)^{2} A^{(2)}\right] .
$$

The Born contribution reads

$$
A^{(0)}=\frac{1}{2 s_{\mathrm{W}}^{2}}
$$


At one loop, the NLL part consists of double- and single-logarithmic terms and reads

$$
A^{(1)} \stackrel{\mathrm{NLL}}{=}-\frac{1}{2 s_{\mathrm{W}}^{2}}\left[C_{q_{\mathrm{L}}}^{\mathrm{ew}}\left(\mathrm{L}_{\hat{s}}^{2}-3 \mathrm{~L}_{\hat{s}}\right)+\frac{C_{\mathrm{A}}}{2 s_{\mathrm{W}}^{2}}\left(\mathrm{~L}_{\hat{t}}^{2}+\mathrm{L}_{\hat{u}}^{2}-\mathrm{L}_{\hat{s}}^{2}\right)\right] .
$$

Here we used the shorthand $\mathrm{L}_{\hat{r}}^{k}=\ln ^{k}\left(|\hat{r}| / M_{W}^{2}\right)$ for the logarithms and $C_{q_{\mathrm{L}}}^{\mathrm{ew}}=$ $Y_{q_{\mathrm{L}}}^{2} /\left(4 c_{\mathrm{W}}^{2}\right)+C_{\mathrm{F}} / s_{\mathrm{W}}^{2}$ are the eigenvalues of the electroweak Casimir operator for lefthanded quarks. This expression is consistent with the process-independent results of Ref. [14] as well as with the NLL part of the one-loop asymptotic expressions presented in Sect. 4.1. At two loops we obtain

$$
\begin{aligned}
A^{(2) \stackrel{\mathrm{NLL}}{=}} & \frac{1}{2 s_{\mathrm{W}}^{2}}\left\{\frac{1}{2}\left(C_{q_{\mathrm{L}}}^{\mathrm{ew}}+\frac{C_{\mathrm{A}}}{2 s_{\mathrm{W}}^{2}}\right)\left[C_{q_{\mathrm{L}}}^{\mathrm{ew}}\left(\mathrm{L}_{\hat{s}}^{4}-6 \mathrm{~L}_{\hat{s}}^{3}\right)+\frac{C_{\mathrm{A}}}{2 s_{\mathrm{W}}^{2}}\left(\mathrm{~L}_{\hat{t}}^{4}+\mathrm{L}_{\hat{u}}^{4}-\mathrm{L}_{\hat{s}}^{4}\right)\right]\right. \\
& \left.+\frac{1}{6}\left[\frac{b_{1}}{c_{\mathrm{W}}^{2}}\left(\frac{Y_{q_{\mathrm{L}}}}{2}\right)^{2}+\frac{b_{2}}{s_{\mathrm{W}}^{2}}\left(C_{\mathrm{F}}+\frac{C_{\mathrm{A}}}{2}\right)\right] \mathrm{L}_{\hat{s}}^{3}\right\},
\end{aligned}
$$

where $b_{1}=-41 /\left(6 c_{\mathrm{w}}^{2}\right)$ and $b_{2}=19 /\left(6 s_{\mathrm{w}}^{2}\right)$ are the one-loop $\beta$-function coefficients associated with the $\mathrm{U}(1)$ and $\mathrm{SU}(2)$ couplings, respectively. The LLs as well as the angular-dependent subset of the NLLs in (87), i.e. all contributions of the form $\mathrm{L}_{\hat{r}}^{4}$ with $\hat{r}=\hat{s}, \hat{t}, \hat{u}$, have been derived from Ref. [15]. There, by means of a diagrammatic two-loop calculation in the spontaneously broken electroweak theory, it was shown that such two-loop terms result from the exponentiation of the corresponding one-loop corrections. The additional NLLs of the form $\mathrm{L}_{\hat{s}}^{3}$ in (87) have been obtained via a fixed-order expansion of the process-independent resummed expression proposed in Ref. [16]. This resummation [16] relies on the assumption that effects from spontaneous breaking of the $\mathrm{SU}(2) \times \mathrm{U}(1)$ symmetry can be neglected in the high-energy limit.

Our NNLO predictions include only the LL and NLL terms. Thus they are affected by a potentially large theoretical uncertainty, due to missing subleading contributions of order $\alpha^{2} \ln ^{k}\left(\hat{s} / M_{W}^{2}\right)$ with $k=2,1,0$. For four-fermion scattering it was found that, at $\hat{s} \sim 1 \mathrm{TeV}^{2}$, the two-loop logarithmic expansion has an oscillating behaviour characterized by large cancellations between leading and subleading terms [3]. In this case the subleading terms play a very important role and the NLL approximation yields misleading results. In contrast, in the case of $W j$ production, the relative weight of the LL, NLL and NNLL contributions at one loop indicates a fairly good convergence of the logarithmic expansion. Indeed, as can be seen from our numerical results in Sect. 7, the one-loop corrections are clearly dominated by the negative LL contributions, while the NLL terms are relatively small and the NNLL contributions almost negligible. A similar convergence is expected also at two-loops, owing to the exponentiation property of the logarithmic corrections. Thus our NLL two-loop predictions can be regarded as a plausible estimate of the size of the two-loop electroweak effects in high- $p_{\mathrm{T}} W$-boson production. 


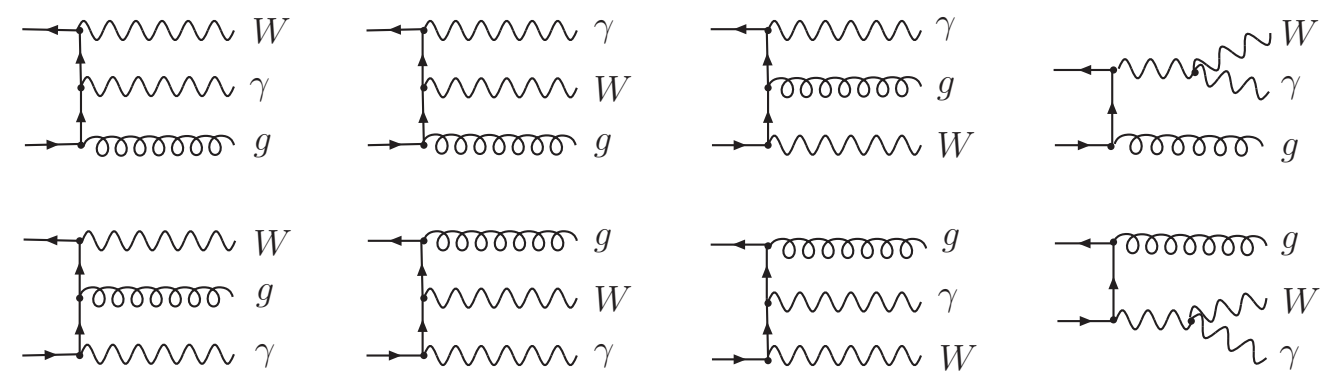

Figure 4: Tree-level diagrams for the process $\bar{q} q^{\prime} \rightarrow W g \gamma$.

\section{Real corrections}

In order to cancel IR singularities from the virtual-photon corrections, real emission corrections need to be calculated. As discussed in Sect. 2.3, all relevant partonic reactions are related to the $\bar{q} q^{\prime} \rightarrow W^{\sigma} g \gamma$ process through crossing and CP symmetry. The tree-level diagrams for this process are shown in Fig. 4 .

The squared matrix element for the $2 \rightarrow 3$ process $a b \rightarrow W^{\sigma} k \gamma$, summed over polarization and colour as well as averaged over initial-state polarization, can be written in a general form 29

$$
\begin{aligned}
\bar{\sum}\left|\mathcal{M}_{0}^{a b \rightarrow W^{\sigma} k \gamma}\right|^{2} & =4 \pi \alpha\left[-Q_{q} Q_{q^{\prime}} H_{\mathrm{r}}^{1, a b}\left(\hat{s}, \hat{t}, \hat{u}, \hat{t}^{\prime}, \hat{u}^{\prime}\right)\right. \\
& \left.+\sigma Q_{q} H_{\mathrm{r}}^{2, a b}\left(\hat{s}, \hat{t}, \hat{u}, \hat{t}^{\prime}, \hat{u}^{\prime}\right)-\sigma Q_{q^{\prime}} H_{\mathrm{r}}^{3, a b}\left(\hat{s}, \hat{t}, \hat{u}, \hat{t}^{\prime}, \hat{u}^{\prime}\right)\right]
\end{aligned}
$$

with the kinematical invariants defined in Sect. 2.2. In the limit of soft and/or collinear photon emission, the squared matrix element (88) exhibits IR singularities. To combine these singularities with those originating from virtual corrections we have to extract them in analytic form. This is done with the help of the dipole subtraction formalism [17, 18, 19]. Within this framework the partonic differential cross section can be schematically written as

$$
\frac{\mathrm{d} \hat{\sigma}^{a b \rightarrow W^{\sigma} k \gamma}}{\mathrm{d} p_{\mathrm{T}}}=\mathcal{N}_{a b} \int \mathrm{d} \Phi_{3}\left[\mathbf{M}^{a b}\left(\Phi_{3}\right)-\mathbf{M}_{\mathrm{sub}}^{a b}\left(\Phi_{3}\right)\right]+\frac{\mathrm{d} \hat{\sigma}_{\mathrm{A}}^{a b}}{\mathrm{~d} p_{\mathrm{T}}},
$$

with $\mathcal{N}_{a b}$ given in (7). The quantity $\mathrm{M}^{a b}$ reads

$$
\mathrm{M}^{a b}\left(\Phi_{3}\right)=\bar{\sum}\left|\mathcal{M}_{0}^{a b \rightarrow W^{\sigma} k \gamma}\right|^{2} F_{\mathrm{O}, 3}\left(\Phi_{3}\right)
$$

The auxiliary function $\mathbf{M}_{\text {sub }}^{a b}$ is chosen such that it has the same singular behaviour as $\mathbf{M}^{a b}$ in the soft and collinear limits. This ensures that the difference $\mathbf{M}^{a b}-\mathbf{M}_{\text {sub }}^{a b}$ can be integrated numerically. To compensate for the subtraction, the integral of the auxiliary function $\mathrm{M}_{\text {sub }}^{a b}$, denoted here $\mathrm{d} \hat{\sigma}_{\mathrm{A}}^{a b} / \mathrm{d} p_{\mathrm{T}}$, is then added back. The analytical form of $\mathrm{d} \hat{\sigma}_{\mathrm{A}}^{a b} / \mathrm{d} p_{\mathrm{T}}$ is obtained after performing integration over the subspace of the 
radiated photon. The result of this one-particle subspace integration contains singular contributions which must be combined with those in virtual corrections. The algorithms for constructing the auxiliary subtraction function and its integrated counterpart have been developed both for the case of photon radiation off massless or massive fermions [17] and QCD radiation off massless [18] or massive partons [19]. In Sects. 5.1 and 5.2 we discuss the application of both formalisms to calculate the $\mathcal{O}(\alpha)$ real corrections to the $W j$ production process. In both approaches we use expressions for the emission off a massive fermion to describe the emission off a $W$ boson, since only soft singularities are present in this case and they depend only on the charge of the external particle and not on its spin.

After adding the real and virtual corrections, collinear singularities remain. Final-state singularities are avoided by recombining collinear photon-quark configurations as discussed in Sect. 2.2. Initial-state singularities are absorbed in the definition of PDFs using the $\overline{\mathrm{MS}}$ scheme.

\subsection{Mass regularization}

The formalism of Ref. [17] employs small photon and fermion masses to regularize soft and collinear singularities. The subtraction term for the squared matrix element is constructed from the appropriate dipole factors. Keeping the original notation of Ref. [17] we can write for the process $a b \rightarrow W^{\sigma} k \gamma$ (where $a(b)$ can be $\bar{q}, q^{\prime}, g$ )

$$
\begin{aligned}
\mathrm{M}_{\mathrm{sub}}^{a b}\left(\Phi_{3}\right)=-4 \pi \alpha \sum_{\tau= \pm}\{ & \left(Q_{a} Q_{b} g_{a b, \tau}^{\mathrm{sub}}\left(p_{a}, p_{b}, p_{\gamma}\right) \mathrm{M}_{0}^{a^{\prime} b^{\prime}}\left(\tilde{\Phi}_{2, a b}\right)\right. \\
& -Q_{a} \sigma g_{a W, \tau}^{\mathrm{sub}}\left(p_{a}, p_{W}, p_{\gamma}\right) \mathrm{M}_{0}^{a^{\prime} b^{\prime}}\left(\tilde{\Phi}_{2, a W}\right) \\
& -\sigma Q_{a} g_{W a, \tau}^{\mathrm{sub}}\left(p_{W}, p_{a}, p_{\gamma}\right) \mathrm{M}_{0}^{a^{\prime} b^{\prime}}\left(\tilde{\Phi}_{2, W a}\right) \\
& -Q_{a} Q_{k} g_{a k, \tau}^{\mathrm{sub}}\left(p_{a}, p_{k}, p_{\gamma}\right) \mathrm{M}_{0}^{a^{\prime} b^{\prime}}\left(\tilde{\Phi}_{2, a k}\right) \\
& \left.-Q_{k} Q_{a} g_{k a, \tau}^{\mathrm{sub}}\left(p_{k}, p_{a}, p_{\gamma}\right) \mathrm{M}_{0}^{a^{\prime} b^{\prime}}\left(\tilde{\Phi}_{2, k a}\right)+(a \leftrightarrow b)\right)\left.\right|_{\left\{a^{\prime}=a, b^{\prime}=b\right\}} \\
& +Q_{k} \sigma g_{k W, \tau}^{\mathrm{sub}}\left(p_{k}, p_{W}, p_{\gamma}\right) \mathrm{M}_{0}^{a b}\left(\tilde{\Phi}_{2, k W}\right) \\
& \left.+\sigma Q_{k} g_{W k, \tau}^{\mathrm{sub}}\left(p_{W}, p_{k}, p_{\gamma}\right) \mathrm{M}_{0}^{a b}\left(\tilde{\Phi}_{2, W k}\right)\right\}
\end{aligned}
$$

with

$$
\mathrm{M}_{0}^{a b}\left(\tilde{\Phi}_{2, n m}\right)=\bar{\sum}\left|\mathcal{M}_{0}^{a b \rightarrow W^{\sigma} k}\left(\tilde{\Phi}_{2, n m}\right)\right|^{2} F_{\mathrm{O}, 2}\left(\tilde{\Phi}_{2, n m}\right) .
$$

Due to $Q_{g}=0$ the dipole terms with gluon indices do not contribute to (91) and for each subprocess the subtraction term $\mathbf{M}_{\text {sub }}^{a b}$ is constructed from six dipole terms, characterised by the $g^{\text {sub }}$ functions. Expressions for these functions are taken directly from Ref. [17]. In Appendix E (see Table 1) we list all the functions which are used to calculate (91), together with the corresponding equation numbers in Ref. [17]. 
For each subprocess the six dipole terms fall into three groups, each containing two dipole terms and coming with a specific charge combination, either $-Q_{q} Q_{q^{\prime}}$ or $\sigma Q_{q}$, or $-\sigma Q_{q^{\prime}}$. The subtraction term $\mathbf{M}_{\text {sub }}^{a b}$ has then the same structure as $\mathbf{M}^{a b}$ in (88) and the IR-singular part of the virtual corrections (75). Thus the cancellation of singularities can be analyzed for each charge combination separately.

The construction of the reduced phase space $\tilde{\Phi}_{2, n m}$ follows the prescriptions of Ref. [17]. Generally $\tilde{\Phi}_{2, n m}$ is a mapping from the 3 -particle phase space into a 2-particle phase space. The mapping respects all mass shell conditions. For different types of dipoles, different mappings are necessary. In Table 1 we list numbers of equations in Ref. [17] which we used to perform mapping for the dipole terms appearing in our calculations. In particular, the observable-defining function $F_{\mathrm{O}, 2}\left(\tilde{\Phi}_{2, n m}\right)$ in (92) is then

$$
F_{\mathrm{O}, 2}\left(\tilde{\Phi}_{2, n m}\right)=\delta\left(p_{\mathrm{T}}-\tilde{p}_{\mathrm{T}, W}\right) \theta\left(\tilde{p}_{\mathrm{T}, j}-p_{\mathrm{T}, j}^{\min }\right),
$$

with $\tilde{p}_{\mathrm{T}, W}$ and $\tilde{p}_{\mathrm{T}, j}$ belonging to $\tilde{\Phi}_{2, n m}$.

The expression for the subtraction term integrated over the phase space of the photon reads

$$
\begin{aligned}
& \frac{\mathrm{d} \hat{\sigma}_{\mathrm{A}}^{a b}}{\mathrm{~d} p_{\mathrm{T}}}=-\frac{\alpha}{2 \pi}\left\{2 Q _ { a } Q _ { b } \quad \left[G_{\mathrm{I}, \mathrm{I}}^{\mathrm{sub}}\left(\hat{r}_{a b}\right) \frac{\mathrm{d} \hat{\sigma}_{\mathrm{fwd}}^{a b \rightarrow W^{\sigma} k}}{\mathrm{~d} p_{\mathrm{T}}}\left(\hat{s}, p_{\mathrm{T}}\right)\right.\right. \\
& \left.+\int_{0}^{1} \mathrm{~d} x\left[\mathcal{G}_{\mathrm{I}, \mathrm{I}}^{\mathrm{sub}}\left(\hat{r}_{a b}, x\right)\right]_{+} \frac{\mathrm{d} \hat{\sigma}_{\mathrm{fwd}}^{a b \rightarrow W^{\sigma} k}}{\mathrm{~d} p_{\mathrm{T}}}\left(x \hat{s}, p_{\mathrm{T}}\right)+(a \leftrightarrow b)\right] \\
& -Q_{a} \sigma \quad\left[G_{\mathrm{I}, \mathrm{FM}}^{\mathrm{sub}}\left(\hat{r}_{a W}\right) \frac{\mathrm{d} \hat{\sigma}_{\mathrm{fwd}}^{a b \rightarrow W^{\sigma} k}}{\mathrm{~d} p_{\mathrm{T}}}\left(\hat{s}, p_{\mathrm{T}}\right)\right. \\
& \left.+\int_{0}^{1} \mathrm{~d} x\left[\mathcal{G}_{\mathrm{I}, \mathrm{FM}}^{\mathrm{sub}}\left(\hat{r}_{a W}(x), x\right)\right]_{+} \frac{\mathrm{d} \hat{\sigma}_{\mathrm{fwd}}^{a b \rightarrow W^{\sigma} k}}{\mathrm{~d} p_{\mathrm{T}}}\left(x \hat{s}, p_{\mathrm{T}}\right)+(a \leftrightarrow b)\right] \\
& -Q_{b} \sigma\left[G_{\mathrm{I}, \mathrm{FM}}^{\mathrm{sub}}\left(\hat{r}_{b W}\right) \frac{\mathrm{d} \hat{\sigma}_{\mathrm{fwd}}^{a b \rightarrow W^{\sigma} k}}{\mathrm{~d} p_{\mathrm{T}}}\left(\hat{s}, p_{\mathrm{T}}\right)\right. \\
& \left.+\int_{0}^{1} \mathrm{~d} x\left[\mathcal{G}_{\mathrm{I}, \mathrm{FM}}^{\mathrm{sub}}\left(\hat{r}_{b W}(x), x\right)\right]_{+} \frac{\mathrm{d} \hat{\sigma}_{\mathrm{fwd}}^{a b \rightarrow W^{\sigma} k}}{\mathrm{~d} p_{\mathrm{T}}}\left(x \hat{s}, p_{\mathrm{T}}\right)+(a \leftrightarrow b)\right] \\
& -Q_{a} Q_{k}\left[G_{\mathrm{I}, \mathrm{F}}^{\mathrm{sub}}\left(\hat{r}_{a k}\right) \frac{\mathrm{d} \hat{\sigma}_{\mathrm{fwd}}^{a b \rightarrow W^{\sigma} k}}{\mathrm{~d} p_{\mathrm{T}}}\left(\hat{s}, p_{\mathrm{T}}\right)\right. \\
& \left.+\int_{0}^{1} \mathrm{~d} x\left[\mathcal{G}_{\mathrm{I}, \mathrm{F}}^{\mathrm{sub}}\left(\hat{r}_{a k}(x), x\right)\right]_{+} \frac{\mathrm{d} \hat{\sigma}_{\mathrm{fwd}}^{a b \rightarrow W^{\sigma} k}}{\mathrm{~d} p_{\mathrm{T}}}\left(x \hat{s}, p_{\mathrm{T}}\right)+(a \leftrightarrow b)\right] \\
& -Q_{b} Q_{k}\left[G_{\mathrm{I}, \mathrm{F}}^{\mathrm{sub}}\left(\hat{r}_{b k}\right) \frac{\mathrm{d} \hat{\sigma}_{\mathrm{fwd}}^{a b \rightarrow W^{\sigma} k}}{\mathrm{~d} p_{\mathrm{T}}}\left(\hat{s}, p_{\mathrm{T}}\right)\right. \\
& \left.+\int_{0}^{1} \mathrm{~d} x\left[\mathcal{G}_{\mathrm{I}, \mathrm{F}}^{\mathrm{sub}}\left(\hat{r}_{b k}(x), x\right)\right]_{+} \frac{\mathrm{d} \hat{\sigma}_{\mathrm{fwd}}^{a b \rightarrow W^{\sigma} k}}{\mathrm{~d} p_{\mathrm{T}}}\left(x \hat{s}, p_{\mathrm{T}}\right)+(a \leftrightarrow b)\right] \\
& \left.+Q_{k} \sigma\left[G_{\mathrm{F}, \mathrm{FM}}^{\mathrm{sub}}\left(\hat{r}_{k W}\right) \frac{\mathrm{d} \hat{\sigma}_{\mathrm{fwd}}^{a b \rightarrow W^{\sigma} k}}{\mathrm{~d} p_{\mathrm{T}}}\left(\hat{s}, p_{\mathrm{T}}\right)+(a \leftrightarrow b)\right]\right\} \text {. }
\end{aligned}
$$


The relevant invariants in (94) are defined as $\hat{r}_{a b}=\left(p_{a}+p_{b}\right)^{2}=\hat{s}, \hat{r}_{k W}=\left(p_{k}+p_{W}\right)^{2}=$ $\hat{s}$, and

$$
\begin{aligned}
\hat{r}_{a W}(x) & =\left(x p_{a}-p_{W}\right)^{2}, \\
\hat{r}_{a k}(x) & =\left(x p_{a}-p_{k}\right)^{2}, \\
\hat{r}_{n l} & =\hat{r}_{n l}(1) .
\end{aligned}
$$

The terms proportional to $\mathrm{d} \hat{\sigma}_{\mathrm{fwd}}^{a b \rightarrow W^{\sigma} k} / \mathrm{d} p_{\mathrm{T}}$ in (94) represent the contributions originating from the forward hemisphere in the 2-particle phase space [see (10)-(11)]. The $(a \leftrightarrow b)$ terms are the contributions from the backward hemisphere, and

$$
\left.\frac{\mathrm{d} \hat{\sigma}_{\mathrm{fwd}}^{a b \rightarrow W^{\sigma} k}}{\mathrm{~d} p_{\mathrm{T}}}\right|_{a \leftrightarrow b}=\frac{\mathrm{d} \hat{\sigma}_{\mathrm{bkwd}}^{a b \rightarrow W^{\sigma} k}}{\mathrm{~d} p_{\mathrm{T}}} .
$$

Note that the first argument of $\mathrm{d} \hat{\sigma}_{\mathrm{fwd}}^{a b \rightarrow W^{\sigma} k} / \mathrm{d} p_{\mathrm{T}}$ in (94) directly indicates the $x$ dependence of the actual values of the $\hat{t}, \hat{u}$ invariants defined in (5). The plusdistributions appearing in eq. (94) are evaluated according to the prescription

$$
\begin{array}{rl}
\int_{0}^{1} & \mathrm{~d} x\left[\mathcal{G}^{\mathrm{sub}}(\hat{r}(x), x)\right]_{+} \frac{\mathrm{d} \hat{\sigma}}{\mathrm{d} p_{\mathrm{T}}}\left(x \hat{s}, p_{\mathrm{T}}\right)= \\
\quad=\int_{0}^{1} \mathrm{~d} x\left[\mathcal{G}^{\mathrm{sub}}(\hat{r}(x), x) \frac{\mathrm{d} \hat{\sigma}}{\mathrm{d} p_{\mathrm{T}}}\left(x \hat{s}, p_{\mathrm{T}}\right) \theta(x-\hat{\tau})-\mathcal{G}^{\mathrm{sub}}(\hat{r}(1), x) \frac{\mathrm{d} \hat{\sigma}}{\mathrm{d} p_{\mathrm{T}}}\left(\hat{s}, p_{\mathrm{T}}\right)\right],
\end{array}
$$

where $\hat{\tau}=\left(p_{\mathrm{T}}+\sqrt{p_{\mathrm{T}}^{2}+M_{W}^{2}}\right)^{2} / \hat{s}$ guarantees the minimal center-of-mass energy to produce the final state. The expressions for the functions $G^{\text {sub }}$ and $\mathcal{G}^{\text {sub }}$ follow directly 5 from the results in Ref. [17]. For the functions $G^{\text {sub }}$ they read

$$
\begin{aligned}
G_{\mathrm{I}, \mathrm{I}}^{\mathrm{sub}}(\hat{r})= & \frac{1}{2}\left[\operatorname{Re}\left(f_{1, \mathrm{MR}}^{\mathrm{IR}}\right)+\ln ^{2}\left(\frac{\hat{r}}{M_{W}^{2}}\right)-3 \ln \left(\frac{\hat{r}}{M_{W}^{2}}\right)-\frac{2}{3} \pi^{2}+4\right], \\
G_{\mathrm{I}, \mathrm{FM}}^{\mathrm{sub}}(\hat{r})= & \left.\operatorname{Re}\left(f_{2, \mathrm{MR}}^{\mathrm{IR}}\right)\right|_{\hat{t} \leftrightarrow \hat{r}}+2 \ln ^{2}\left(1-\frac{\hat{r}}{M_{W}^{2}}\right)-\ln ^{2}\left(2-\frac{\hat{r}}{M_{W}^{2}}\right) \\
& +\ln \left(1-\frac{\hat{r}}{M_{W}^{2}}\right)\left(\frac{M_{W}^{4}}{\hat{r}^{2}}-\frac{3 M_{W}^{2}}{\hat{r}}-3\right)-2 \operatorname{Li}_{2}\left(\frac{M_{W}^{2}}{2 M_{W}^{2}-\hat{r}}\right) \\
& +2 \operatorname{Li}_{2}\left(\frac{\hat{r}}{2 M_{W}^{2}-\hat{r}}\right)-2 \operatorname{Li}_{2}\left(\frac{-\hat{r}}{2 M_{W}^{2}-\hat{r}}\right)+\frac{M_{W}^{2}}{\hat{r}}+\frac{\pi^{2}}{6}+\frac{1}{2}, \\
G_{\mathrm{I}, \mathrm{F}}^{\mathrm{sub}}(\hat{r})= & \left.\operatorname{Re}\left(f_{1, \mathrm{MR}}^{\mathrm{IR}}\right)\right|_{\hat{s} \leftrightarrow \hat{r}}+\ln ^{2}\left(\frac{-\hat{r}}{M_{W}^{2}}\right)-3 \ln \left(\frac{-\hat{r}}{M_{W}^{2}}\right)-\frac{\pi^{2}}{3}+\frac{1}{2}, \\
G_{\mathrm{F}, \mathrm{FM}}^{\mathrm{sub}}(\hat{r})= & \left.\operatorname{Re}\left(f_{2, \mathrm{MR}}^{\mathrm{IR}}\right)\right|_{\hat{t} \leftrightarrow \hat{r}}+\ln ^{2}\left(\frac{\hat{r}}{M_{W}^{2}}-1\right)+\ln ^{2}\left(1-\frac{M_{W}^{2}}{\hat{r}}\right)
\end{aligned}
$$

\footnotetext{
${ }^{5}$ The function $G_{\mathrm{F}, \mathrm{FM}}^{\mathrm{sub}}(\hat{r})$ has been derived from eq. (4.10) in Ref. [17] by taking the limit of an infinitesimal quark mass.
} 


$$
\begin{aligned}
& +\frac{1}{2} \ln \left(\frac{\hat{r}}{M_{W}^{2}}\right)-\frac{7}{2} \ln \left(\frac{\hat{r}}{M_{W}^{2}}-1\right)-\frac{3}{2} \ln \left(\frac{\sqrt{\hat{r}}-M_{W}}{\sqrt{\hat{r}}+M_{W}}\right) \\
& +4 \operatorname{Li}_{2}\left(\frac{M_{W}^{2}}{\hat{r}}\right)-4 \operatorname{Li}_{2}\left(\sqrt{\frac{M_{W}^{2}}{\hat{r}}}\right)+\frac{M_{W}^{2}}{2 \hat{r}}-\frac{2 \pi^{2}}{3}+3 .
\end{aligned}
$$

The IR-singular functions $f_{i, \mathrm{MR}}^{\mathrm{IR}}$ cancel against those in the virtual corrections, cf. Sect. 3.4. The explicit forms of the functions $\mathcal{G}^{\text {sub }}$ are

$$
\begin{aligned}
\mathcal{G}_{\mathrm{I}, \mathrm{I}}^{\mathrm{sub}}(\hat{r}, x)= & \chi(x)+\frac{1+x^{2}}{1-x}\left\{\ln \left(\frac{\hat{r}}{\mu_{\mathrm{QED}}^{2}}\right)+2 \ln (1-x)\right\}+1-x \\
\mathcal{G}_{\mathrm{I}, \mathrm{FM}}^{\mathrm{sub}}(\hat{r}, x)= & \chi(x)+\frac{1+x^{2}}{1-x}\left\{\ln \left(\frac{M_{W}^{2}-\hat{r}}{x \mu_{\mathrm{QED}}^{2}}\right)+\ln \left((1-x)\left(1-z_{1}(\hat{r}, x)\right)\right)\right\} \\
& +\frac{z_{1}(\hat{r}, x)-1}{2(1-x)}\left(3+z_{1}(\hat{r}, x)-\frac{4 M_{W}^{2} x}{\left(\hat{r}-M_{W}^{2}\right)(1-x)}\right)+1-x, \\
\mathcal{G}_{\mathrm{I}, \mathrm{F}}^{\mathrm{sub}}(\hat{r}, x)= & \chi(x)+\frac{1+x^{2}}{1-x}\left\{\ln \left(\frac{-\hat{r}}{x \mu_{\mathrm{QED}}^{2}}\right)+\ln (1-x)\right\} \\
& -\frac{3}{2(1-x)}+1-x
\end{aligned}
$$

with

$$
z_{1}(\hat{r}, x)=\frac{M_{W}^{2} x}{M_{W}^{2}-(1-x) \hat{r}}
$$

and

$$
\chi(x)=\frac{1+x^{2}}{1-x}\left\{\ln \left(\frac{\mu_{\mathrm{QED}}^{2}}{m^{2}}\right)-2 \ln (1-x)-1\right\},
$$

where $\mu_{\mathrm{QED}}$ is the factorization scale and $m$ stands for the quark-mass regulator. The functions $\chi(x)$ are singular. These singularities are related to the collinear photon radiation off an initial-state quark and are absorbed in the definition of the PDFs, yielding the hadronic cross section finite. The procedure bears complete analogy to absorbing collinear QCD singularities into the definition of the PDFs. In the $\overline{\mathrm{MS}}$ factorization scheme, the redefinition is achieved by replacing [5]

$$
f_{h, q}\left(x, \mu_{\mathrm{QCD}}^{2}\right) \rightarrow f_{h, q}\left(x, \mu_{\mathrm{QCD}}^{2}, \mu_{\mathrm{QED}}^{2}\right)-\frac{\alpha}{2 \pi} Q_{q}^{2} \int_{x}^{1} \frac{\mathrm{d} z}{z} f_{h, q}\left(\frac{x}{z}, \mu_{\mathrm{QCD}}^{2}, \mu_{\mathrm{QED}}^{2}\right)[\chi(z)]_{+} \cdot
$$

\subsection{Dimensional regularization}

In an independent calculation we used the results of Refs. [18, 19] to evaluate the dipole subtraction terms and their integrated counterparts. The formalism of [18, 19] is concerned with QCD radiation and expressions for dipoles are given as matrices in colour and helicity space. Since we consider photon emission off a fermion line, the 
colour and helicity structure disappears and the dipole matrices reduce to simple expressions. More precisely, to adapt the formalism Refs. [18, 19] for the calculation of QED corrections, we make use of expressions describing gluon radiation off a fermion line in Refs. [18, 19] and replace

$$
\alpha_{\mathrm{S}} \rightarrow \alpha, \mathbf{T}_{i} \rightarrow \sigma_{i} Q_{i}, \quad C_{\mathrm{F}} \rightarrow Q_{i}^{2}, \quad T_{\mathrm{R}} \rightarrow 1, \quad C_{\mathrm{A}} \rightarrow 0
$$

where $\mathbf{T}_{i}$ indicates the colour of the emitting parton, $Q_{i}$ is the electric charge in units of the positron charge for this parton, and $\sigma_{i}=+1(-1)$ for incoming (outgoing) partons. Adopting notation analogous to Refs. [18, 19], the subtraction term for the process $a b \rightarrow W^{\sigma} k \gamma$ can be then written

$$
\begin{aligned}
\mathrm{M}_{\mathrm{sub}}^{a b}\left(\Phi_{3}\right)= & {\left[\mathcal{D}_{\mathrm{QED}}^{a \gamma, b}+\mathcal{D}_{W, \mathrm{QED}}^{a \gamma}+\mathcal{D}_{\gamma W, \mathrm{QED}}^{a}+\mathcal{D}_{k, \mathrm{QED}}^{a \gamma}+\mathcal{D}_{\gamma k, \mathrm{QED}}^{a}+(a \leftrightarrow b)\right] } \\
& +\mathcal{D}_{\gamma k, W, \mathrm{QED}}+\mathcal{D}_{\gamma W, k, \mathrm{QED}}
\end{aligned}
$$

where

$$
\mathcal{D}_{F, \mathrm{QED}}^{I}=\left.F_{\mathrm{O}, 2}\left(\tilde{\Phi}_{2, n m}\right) \mathcal{D}_{F}^{I}\left(p_{W}, p_{k}, p_{\gamma} ; p_{a}, p_{b}\right)\right|_{\text {replacements of eq. (108) }} .
$$

It is understood in eq. (109) that dipole subtraction terms with a gluon index do not contribute to $\mathrm{M}_{\mathrm{sub}}^{a b}$. In a complete analogy to eq. (91), for any initial state $a b$ the expression for $\mathrm{M}_{\mathrm{sub}}^{a b}$ is constructed from six dipole subtraction terms $\mathcal{D}_{F, \mathrm{QED}}^{I}$, each associated with one of the three possible charge combinations $-Q_{q} Q_{q^{\prime}}, \sigma Q_{q}$ or $-\sigma Q_{q^{\prime}}$. The dipole subtraction functions $\mathcal{D}_{F}^{I}$ are taken directly from Refs. [18, 19]. A list of the functions $\mathcal{D}_{F}^{I}$ used to calculate the subtraction term $\mathrm{M}_{\text {sub }}^{a b}$ in (109), together with the corresponding equation numbers in Refs. [18, 19], is presented in Table 2, Appendix E. Additionally, for each dipole subtraction term appearing in (109) we include a description of its type. The mappings from $\Phi_{3}$ to $\tilde{\Phi}_{2, n m}$ agree between the formalism of Refs. [18, 19] and [17. However, for the sake of completeness, Table 2 contains numbers of equations which provide mapping formulae in Refs. [18, 19]. The function $F_{\mathrm{O}, 2}$ in (110) is given by expression (93)).

Moreover, apart from the final-state emitter, final-state spectator case, i.e. the dipoles $\mathcal{D}_{\gamma k, W \text {, QED }}$ and $\mathcal{D}_{\gamma W, k \text {, QED }}$, there is a direct correspondence between the dipole subtraction terms in the two formalisms of the form

$$
\begin{aligned}
& \mathcal{D}_{\mathrm{QED}}^{a \gamma, b} \stackrel{\varepsilon \rightarrow 0}{\rightarrow}-Q_{a} Q_{b} 4 \pi \alpha \sum_{\tau= \pm} g_{a b, \tau}^{\mathrm{sub}}\left(p_{a}, p_{b}, p_{\gamma}\right) \mathrm{M}_{0}^{a b}\left(\tilde{\Phi}_{2, a b}\right), \\
& \mathcal{D}_{W, \mathrm{QED}}^{a \gamma} \stackrel{\varepsilon \rightarrow 0}{\rightarrow} Q_{a} \sigma 4 \pi \alpha \sum_{\tau= \pm} g_{a W, \tau}^{\mathrm{sub}}\left(p_{a}, p_{W}, p_{\gamma}\right) \mathrm{M}_{0}^{a b}\left(\tilde{\Phi}_{2, a W}\right), \\
& \mathcal{D}_{\gamma W, \mathrm{QED}}^{a} \stackrel{\varepsilon \rightarrow 0}{\rightarrow} Q_{a} \sigma 4 \pi \alpha \sum_{\tau= \pm} g_{W a, \tau}^{\mathrm{sub}}\left(p_{W}, p_{a}, p_{\gamma}\right) \mathrm{M}_{0}^{a b}\left(\tilde{\Phi}_{2, W a}\right), \\
& \mathcal{D}_{k, \mathrm{QED}}^{a \gamma} \stackrel{\varepsilon \rightarrow 0}{\rightarrow} Q_{a} Q_{k} 4 \pi \alpha \sum_{\tau= \pm} g_{a k, \tau}^{\mathrm{sub}}\left(p_{a}, p_{k}, p_{\gamma}\right) \mathrm{M}_{0}^{a b}\left(\tilde{\Phi}_{2, a k}\right), \\
& \mathcal{D}_{\gamma k, \mathrm{QED}}^{a} \stackrel{\varepsilon \rightarrow 0}{\rightarrow} Q_{a} Q_{k} 4 \pi \alpha \sum_{\tau= \pm} g_{k a, \tau}^{\mathrm{sub}}\left(p_{k}, p_{a}, p_{\gamma}\right) \mathrm{M}_{0}^{a b}\left(\tilde{\Phi}_{2, k a}\right) .
\end{aligned}
$$


The subtraction term integrated over the photon phase space is constructed according to

$$
\frac{\mathrm{d} \hat{\sigma}_{\mathrm{A}}^{a b}}{\mathrm{~d} p_{\mathrm{T}}}=\frac{\alpha}{2 \pi}\left[\mathcal{I}^{a b}\left(\hat{s}, p_{\mathrm{T}}\right)+\int_{0}^{1} \mathrm{~d} x\left(\mathcal{K}^{a b}\left(x, \hat{s}, p_{\mathrm{T}}\right)+\mathcal{P}^{a b}\left(x, \hat{s}, p_{\mathrm{T}}\right)\right)\right],
$$

where the expressions for $\mathcal{I}, \mathcal{K}$ and $\mathcal{P}$ functions follow from results for the integrated dipole functions in Refs. [18, 19] after performing replacements of eq. (108). For the photonic corrections to any of the subprocesses $a b \rightarrow W^{\sigma} k$ we can write

$$
\begin{aligned}
\mathcal{I}^{a b}\left(\hat{s}, p_{\mathrm{T}}\right)= & Q_{a} Q_{b}\left[\tilde{\mathcal{I}}\left(\hat{s}_{a b}\right) \frac{\mathrm{d} \hat{\sigma}_{\mathrm{fwd}}^{a b \rightarrow W^{\sigma} k}}{\mathrm{~d} p_{\mathrm{T}}}\left(\hat{s}, p_{\mathrm{T}}\right)+(a \leftrightarrow b)\right] \\
& -Q_{a} \sigma\left[\mathcal{I}^{\prime}\left(\hat{s}_{a W}\right) \frac{\mathrm{d} \hat{\sigma}_{\mathrm{fwd}}^{a b \rightarrow} W^{\sigma} k}{\mathrm{~d} p_{\mathrm{T}}}\left(\hat{s}, p_{\mathrm{T}}\right)+(a \leftrightarrow b)\right] \\
& -Q_{b} \sigma\left[\mathcal{I}^{\prime}\left(\hat{s}_{b W}\right) \frac{\mathrm{d} \hat{\sigma}_{\mathrm{fwd}}^{a b \rightarrow W^{\sigma} k}}{\mathrm{~d} p_{\mathrm{T}}}\left(\hat{s}, p_{\mathrm{T}}\right)+(a \leftrightarrow b)\right] \\
& -Q_{a} Q_{k}\left[\tilde{\mathcal{I}}\left(\hat{s}_{a k}\right) \frac{\mathrm{d} \hat{\sigma}_{\mathrm{fwd}}^{a b \rightarrow W^{\sigma} k}}{\mathrm{~d} p_{\mathrm{T}}}\left(\hat{s}, p_{\mathrm{T}}\right)+(a \leftrightarrow b)\right] \\
& -Q_{b} Q_{k}\left[\tilde{\mathcal{I}}\left(\hat{s}_{b k}\right) \frac{\mathrm{d} \hat{\sigma}_{\mathrm{fwd}}^{a b \rightarrow W^{\sigma} k}}{\mathrm{~d} p_{\mathrm{T}}}\left(\hat{s}, p_{\mathrm{T}}\right)+(a \leftrightarrow b)\right] \\
& +Q_{k} \sigma\left[\mathcal{I}^{\prime}\left(\hat{s}_{k W}\right) \frac{\mathrm{d} \hat{\sigma}_{\mathrm{fwd}}^{a b \rightarrow W^{\sigma} k}}{\mathrm{~d} p_{\mathrm{T}}}\left(\hat{s}, p_{\mathrm{T}}\right)+(a \leftrightarrow b)\right],
\end{aligned}
$$

where $\hat{s}_{n m}=2 p_{n} p_{m}$, and we make use of $Q_{g}=0$. As in (94), the terms proportional to $\mathrm{d} \hat{\sigma}_{\text {fwd }}^{a b \rightarrow W^{\sigma} k} / \mathrm{d} p_{\mathrm{T}}$ originate from the forward hemisphere and the $(a \leftrightarrow b)$ terms from the backward hemisphere. The integrated dipole functions in (113) read

$$
\begin{aligned}
\tilde{\mathcal{I}}\left(\hat{s}_{n m}\right)= & -\left.\left(\frac{4 \pi \mu^{2}}{M_{W}^{2}}\right)^{\varepsilon} \Gamma(1+\varepsilon) \operatorname{Re}\left(f_{1, \mathrm{DR}}^{\mathrm{IR}}\right)\right|_{\hat{s}=\hat{s}_{n m}}-\ln ^{2}\left(\frac{\hat{s}_{n m}}{M_{W}^{2}}\right)+3 \ln \left(\frac{\hat{s}_{n m}}{M_{W}^{2}}\right) \\
& +\frac{4 \pi^{2}}{3}-6, \\
\mathcal{I}^{\prime}\left(\hat{s}_{n m}\right)= & -\left.\left(\frac{4 \pi \mu^{2}}{M_{W}^{2}}\right)^{\varepsilon} \Gamma(1+\varepsilon) \operatorname{Re}\left(f_{2, \mathrm{DR}}^{\mathrm{IR}}\right)\right|_{\hat{t}=M_{W}^{2}+\sigma_{n} \sigma_{m} \hat{s}_{n m}}-\ln ^{2}\left(\frac{\hat{s}_{n m}}{M_{W}^{2}}\right) \\
& -\ln \left(\frac{\hat{s}_{n m}}{M_{W}^{2}}\right)\left(\ln \left(\frac{\hat{s}_{n m}}{M_{W}^{2}+\hat{s}_{n m}}\right)-3\right)-\ln \left(\frac{\hat{s}_{n m}}{M_{W}^{2}+\hat{s}_{n m}}\right) \\
& +\ln \left(\frac{M_{W}^{2}}{M_{W}^{2}+\hat{s}_{n m}}\right) \ln \left(\frac{\hat{s}_{n m}}{M_{W}^{2}+\hat{s}_{n m}}\right)+3 \ln \left(1-\sqrt{\frac{M_{W}^{2}}{M_{W}^{2}+\hat{s}_{n m}}}\right) \\
& +\frac{M_{W}^{2}}{\hat{s}_{n m}} \ln \left(\frac{M_{W}^{2}}{M_{W}^{2}+\hat{s}_{n m}}\right)+2 \mathrm{Li}_{2}\left(\frac{\hat{s}_{n m}}{M_{W}^{2}+\hat{s}_{n m}}\right)+\frac{3 M_{W}}{\sqrt{\hat{s}_{n m}+M_{W}^{2}}+M_{W}} \\
& +\pi^{2}-6 .
\end{aligned}
$$


The structure of the singular terms $f_{i, \mathrm{DR}}^{\mathrm{IR}}$ in (114) is kept the same as in (75) to manifestly show cancellation of singularities between virtual and real corrections. For the $x$-dependent functions we have

$$
\begin{aligned}
\mathcal{K}^{a b}\left(x, \hat{s}, p_{\mathrm{T}}\right)= & \left(Q_{a}^{2}+Q_{b}^{2}\right)\left[\overline{\mathcal{K}}(x) \frac{\mathrm{d} \hat{\sigma}_{\mathrm{fwd}}^{a b \rightarrow W^{\sigma} k}}{\mathrm{~d} p_{\mathrm{T}}}\left(x \hat{s}, p_{\mathrm{T}}\right)+(a \leftrightarrow b)\right] \\
& +2 Q_{a} Q_{b}\left[\tilde{\mathcal{K}}(x) \frac{\mathrm{d} \hat{\sigma}_{\mathrm{fwd}}^{a b \rightarrow W^{\sigma} k}}{\mathrm{~d} p_{\mathrm{T}}}\left(x \hat{s}, p_{\mathrm{T}}\right)+(a \leftrightarrow b)\right] \\
& -Q_{a} \sigma\left[\mathcal{K}^{\prime}\left(x, \hat{s}_{a W}\right) \frac{\mathrm{d} \hat{\sigma}_{\mathrm{fwd}}^{a b \rightarrow W^{\sigma} k}}{\mathrm{~d} p_{\mathrm{T}}}\left(x \hat{s}, p_{\mathrm{T}}\right)+(a \leftrightarrow b)\right] \\
& -Q_{b} \sigma\left[\mathcal{K}^{\prime}\left(x, \hat{s}_{b W}\right) \frac{\mathrm{d} \hat{\sigma}_{\mathrm{fwd}}^{a b \rightarrow W^{\sigma} k}}{\mathrm{~d} p_{\mathrm{T}}}\left(x \hat{s}, p_{\mathrm{T}}\right)+(a \leftrightarrow b)\right] \\
& -\left(Q_{a} Q_{k}+Q_{b} Q_{k}\right)\left[\mathcal{K}^{\prime \prime}(x) \frac{\mathrm{d} \hat{\sigma}_{\mathrm{fwd}}^{a b \rightarrow W^{\sigma} k}}{\mathrm{~d} p_{\mathrm{T}}}\left(x \hat{s}, p_{\mathrm{T}}\right)+(a \leftrightarrow b)\right], \\
\mathcal{P}^{a b}\left(x, \hat{s}, p_{\mathrm{T}}\right)= & 2 Q_{a} Q_{b}\left[\mathcal{P}\left(x, \hat{s}_{a b}\right) \frac{\mathrm{d} \hat{\sigma}_{\mathrm{fwd}}^{a b \rightarrow} W^{\sigma} k}{\mathrm{~d} p_{\mathrm{T}}}\left(x \hat{s}, p_{\mathrm{T}}\right)+(a \leftrightarrow b)\right] \\
& -Q_{a} \sigma\left[\mathcal{P}\left(x, \hat{s}_{a W}\right) \frac{\mathrm{d} \hat{\mathrm{d}}_{\mathrm{fwd}}^{a b \rightarrow W^{\sigma} k}}{\mathrm{~d} p_{\mathrm{T}}}\left(x \hat{s}, p_{\mathrm{T}}\right)+(a \leftrightarrow b)\right] \\
& -Q_{b} \sigma\left[\mathcal{P}\left(x, \hat{s}_{b W}\right) \frac{\mathrm{d} \hat{\sigma}_{\mathrm{fwd}}^{\hat{f}^{\prime a \rightarrow} W^{\sigma} k}}{\mathrm{~d} p_{\mathrm{T}}}\left(x \hat{s}, p_{\mathrm{T}}\right)+(a \leftrightarrow b)\right] \\
& -Q_{a} Q_{k}\left[\mathcal{P}\left(x, \hat{s}_{a k}\right) \frac{\mathrm{d} \hat{\sigma}_{\mathrm{fwd}}^{a b \rightarrow W^{\sigma} k}}{\mathrm{~d} p_{\mathrm{T}}}\left(x \hat{s}, p_{\mathrm{T}}\right)+(a \leftrightarrow b)\right] \\
& -Q_{b} Q_{k}\left[\mathcal{P}\left(x, \hat{s}_{b k}\right) \frac{\mathrm{d} \hat{\sigma}_{\mathrm{fwd}}^{a b \rightarrow W^{\sigma} k}}{\mathrm{~d} p_{\mathrm{T}}}\left(x \hat{s}, p_{\mathrm{T}}\right)+(a \leftrightarrow b)\right],
\end{aligned}
$$

with

$$
\begin{aligned}
\overline{\mathcal{K}}(x)= & P_{\text {reg }}(x) \ln \left(\frac{1-x}{x}\right)+(1-x)+\left(\frac{2}{1-x} \ln \left(\frac{1-x}{x}\right)\right)_{+} \\
& -\delta(1-x)\left(5-\pi^{2}\right), \\
\tilde{\mathcal{K}}(x)= & -P_{\operatorname{reg}}(x) \ln (1-x)-\left[2\left(\frac{\ln (1-x)}{1-x}\right)_{+}-\frac{\pi^{2}}{3} \delta(1-x)\right], \\
\mathcal{K}^{\prime}\left(x, \hat{s}_{n m}\right)= & -2\left(\frac{\ln (1-x)}{1-x}\right)_{+}+2 \frac{\ln (2-x)}{1-x} \\
& -\left(\frac{1-x}{2\left(1-x+M_{W}^{2} / \hat{s}_{n m}\right)^{2}}-\frac{2}{1-x}\left[1+\ln \left(1-x+\frac{M_{W}^{2}}{\hat{s}_{n m}}\right)\right]\right)_{+} \\
& -\left(\frac{2}{1-x}\right)_{+}\left[\ln \left(2-x+\frac{M_{W}^{2}}{\hat{s}_{n m}}\right)+\ln \left(\frac{(2-x) \hat{s}_{n m}}{(2-x) \hat{s}_{n m}+M_{W}^{2}}\right)\right] \\
& -P_{\text {reg }}(x) \ln \left(\frac{(1-x) \hat{s}_{n m}}{(1-x) \hat{s}_{n m}+M_{W}^{2}}\right)
\end{aligned}
$$




$$
\begin{aligned}
& -\delta(1-x)\left[-\frac{3}{2}+\frac{M_{W}^{2}}{\hat{s}_{n m}} \ln \left(\frac{M_{W}^{2}}{\hat{s}_{n m}+M_{W}^{2}}\right)+\frac{3 M_{W}}{\sqrt{\hat{s}_{n m}+M_{W}^{2}}+M_{W}}\right. \\
& \left.+\frac{3}{2} \ln \left(\frac{\hat{s}_{n m}-2 M_{W} \sqrt{\hat{s}_{n m}+M_{W}^{2}}+2 M_{W}^{2}}{\hat{s}_{n m}}\right)+\frac{1}{2} \frac{M_{W}^{2}}{\hat{s}_{n m}+M_{W}^{2}}\right] \\
\mathcal{K}^{\prime \prime}(x)= & \frac{3}{2}\left[\left(\frac{1}{1-x}\right)_{+}+\delta(1-x)\right] \\
\mathcal{P}\left(x, \hat{s}_{n m}\right)= & \left(\frac{1+x^{2}}{1-x}\right)_{+} \ln \left(\frac{\mu_{\mathrm{QED}}^{2}}{x \hat{s}_{n m}}\right)
\end{aligned}
$$

and

$$
P_{\mathrm{reg}}(x)=\left(\frac{1+x^{2}}{1-x}\right)_{+}-\left(\frac{2}{1-x}\right)_{+}-\frac{3}{2} \delta(1-x) .
$$

Note that in contrast to eq. (113), the quantity $\hat{s}_{n m}$ in (115) can be implicitly dependent on the fraction $x$. More precisely, it is the case if $\hat{s}_{n m}$ involves the momentum of a final-state particle. The final-state momentum belongs then to the phase space for which the squared center-of-mass energy is $x \hat{s}=2 x p_{a} p_{b}$ [19].

The evaluation of the terms involving the plus-distribution is carried out as indicated in Ref. [19], i.e. according to

$$
\begin{aligned}
& \int_{0}^{1} \mathrm{~d} x {\left[\mathcal{R}\left(x, \hat{s}_{n m}(x)\right)\right]_{+} \frac{\mathrm{d} \hat{\sigma}}{\mathrm{d} p_{\mathrm{T}}}\left(x \hat{s}, p_{\mathrm{T}}\right)=} \\
& \quad=\int_{0}^{1} \mathrm{~d} x\left[\mathcal{R}\left(x, \hat{s}_{n m}(x)\right) \frac{\mathrm{d} \hat{\sigma}}{\mathrm{d} p_{\mathrm{T}}}\left(x \hat{s}, p_{\mathrm{T}}\right) \theta(x-\hat{\tau})-\mathcal{R}\left(x, \hat{s}_{n m}(1)\right) \frac{\mathrm{d} \hat{\sigma}}{\mathrm{d} p_{\mathrm{T}}}\left(\hat{s}, p_{\mathrm{T}}\right)\right],
\end{aligned}
$$

with $\hat{\tau}=\left(p_{\mathrm{T}}+\sqrt{p_{\mathrm{T}}^{2}+M_{W}^{2}}\right)^{2} / \hat{s}$.

In the formalism of Refs. [18, 19] the collinear counterterms associated with PDF renormalization are included in the expressions for integrated dipole functions, i.e. the final results which we use are free from collinear singularities. The expressions presented here are calculated using the $\overline{\mathrm{MS}}$ factorization scheme.

As can be seen from the presented formulae, the explicit expressions for the integrated dipole functions in the two formalisms are different. In particular, the expressions for the end-point contributions have different forms due to specific conventions wrt. calculating the plus-distribution terms in the two formalisms. However we have checked that, after subtraction of the IR singularities, for each charge combination apart from $\sigma Q_{k}$ the integrated dipole contributions to $\mathrm{d} \sigma_{\mathrm{A}}^{a b} / \mathrm{d} p_{\mathrm{T}}$ in the two formalisms are equivalent. 


\section{Checks}

Every part of the presented calculation has been performed in two completely independent ways. The algebraic reductions were done using two different MATHEMATICA [30] codes. For the numerical evaluation we have implemented the results in two independent ForTRAN programs. Comparing the results at numerical level we find agreement within the statistical errors.

Furthermore, in order to control the correctness of our results we performed various consistency checks. On the side of virtual corrections we have verified that the one-loop corrections (48) satisfy the Ward Identity

$$
\varepsilon_{\mu}^{*}\left(p_{W}\right) p_{g \nu} \bar{v}\left(p_{\bar{q}}\right)\left[\delta \mathcal{A}_{1, \mathrm{I}}^{\mu \nu}\left(M_{V}^{2}\right) \omega_{\lambda}\right] u\left(p_{q}\right)=0 \quad \text { for } \mathrm{I}=\mathrm{A}, \mathrm{N}, \mathrm{X}, \mathrm{Y} .
$$

A similar Ward identity holds for the lowest-order amplitude6. The cancellation of the ultraviolet divergencies has been verified analytically and numerically. For the numerical evaluation of the loop integrals we use a set of routines by A. Denner and, alternatively, the FF library [31]. The NLL approximation that was derived from the full one-loop calculation, has been checked against results from the general derivation of NLL terms [14]. Also the IR-singular contributions in the high-energy limit have been reproduced within this framework.

The squared matrix element for the real corrections was checked numerically against MADGRAPH [32]. The cancellation of IR singularities between real and virtual corrections was done analytically using the dipole formalism. The subtraction terms were derived and implemented in two different ways, using the mass regularization of IR singularities and the dimensional regularization. The phase-space integration for the real corrections was performed with adaptive Monte-Carlo integration using VEGAS [33]. Detailed comparisons at analytical and numerical level were performed, and the agreement between the predictions generated within two different regularization schemes provided a strong check on the calculation of the real corrections.

\section{Numerical results}

In this section we present numerical predictions for the large- $p_{\mathrm{T}}$ production of $W$ bosons at the hadron colliders LHC and Tevatron. The following input parame-

\footnotetext{
${ }^{6}$ We note that the abelian one-loop contribution satisfies two additional Ward identities

$$
p_{W \mu} \varepsilon_{\nu}^{*}\left(p_{g}\right) \bar{v}\left(p_{\bar{q}}\right)\left[\delta \mathcal{A}_{1, \mathrm{~A}}^{\mu \nu}\left(M_{V}^{2}\right) \omega_{\lambda}\right] u\left(p_{q}\right)=0, \quad p_{W \mu} p_{g \nu} \bar{v}\left(p_{\bar{q}}\right)\left[\delta \mathcal{A}_{1, \mathrm{~A}}^{\mu \nu}\left(M_{V}^{2}\right) \omega_{\lambda}\right] u\left(p_{q}\right)=0 .
$$
}

Similar identities for the N-, X- and Y- form factors exist but are less trivial due to the nonvanishing contributions from would-be Goldstone bosons on the right-hand side. This means that the calculation of the unpolarized cross section requires the use of the exact expression for the $W$ boson polarization sum. Instead, owing to (119), the gluon polarization sum can be implemented as $-g_{\nu \nu^{\prime}}$. 
ters are used: $G_{\mu}=1.16637 \times 10^{-5} \mathrm{GeV}^{-2}, M_{W}=80.39 \mathrm{GeV}, M_{Z}=91.19 \mathrm{GeV}$, $m_{t}=171.4 \mathrm{GeV}, M_{H}=120 \mathrm{GeV}$. For the numerical values of elements in the CKM quark mixing matrix we refer to [34].

The hadronic cross sections are obtained using LO MRST2001 PDFs [35]. We choose $\mu_{\mathrm{QCD}}^{2}=p_{\mathrm{T}}^{2}$ as the factorization scale and, similarly, as the scale at which the strong coupling constant is evaluated 7 . We also adopt, in agreement with the value used in the PDF analysis, the value $\alpha_{\mathrm{S}}\left(M_{Z}^{2}\right)=0.13$ and use the one-loop running expression for $\alpha_{\mathrm{S}}\left(\mu_{\mathrm{QCD}}^{2}\right)$. In our calculations of the real corrections we choose the $\overline{\mathrm{MS}}$ factorization scheme with the scale $\mu_{\mathrm{QED}}^{2}=M_{W}^{2}$. We note that in order to consistently include $\mathcal{O}(\alpha)$ corrections in a calculation of a hadronic cross section, PDFs that are used in the calculation need to take into account QED effects. Such PDF analysis has been performed in [36] and the $\mathcal{O}(\alpha)$ effects are known to be small for $\mu_{\mathrm{QED}} \lesssim 100 \mathrm{GeV}$, both concerning the change in the quark distribution functions (below $\mathcal{O}(1 \%)$ [37]) and the size of the photon distribution function. Moreover, the currently available PDFs incorporating $\mathcal{O}(\alpha)$ corrections, MRST2004QED [36], include QCD effects at the NLO in $\alpha_{\mathrm{S}}$. Since our calculations are of the lowest order in QCD, and QED effects on PDFs are estimated to be small for $\mu_{\mathrm{QED}} \lesssim 100 \mathrm{GeV}$, we prefer to use a LO QCD PDF set without QED corrections incorporated, rather than MRST2004QED, and we set $\mu_{\mathrm{QED}}=M_{W} 8$. Moreover we do not include photon-induced contributions, which are parametrically suppressed by a factor $\alpha / \alpha_{\mathrm{S}}$. However, in the concurrent to this paper (and subsequent to Ref. [21]), work of Ref. [22], it has been reported that photon-induced contributions are of numerical significance for large $p_{\mathrm{T}} W$-boson production at the LHC. Estimates of the exact size of these effects are obscured by large theoretical uncertainty on the photon's PDF, as demonstrated in Ref. [22].

We choose the following values of the $p_{\mathrm{T}^{-} \text {cuts: }} p_{\mathrm{T}, j}^{\min }=100 \mathrm{GeV}$ for LHC and $p_{\mathrm{T}, j}^{\min }=50 \mathrm{GeV}$ for Tevatron. The value of the separation parameter below which the recombination procedure is applied is taken to be $R_{\text {sep }}=0.4$. The dependence of our predictions on $R_{\text {sep }}$ is negligible. We have verified that the shift of the transverse-

\footnotetext{
${ }^{7}$ Note that when calculating the contribution to the hadronic cross section coming from the subtraction term in the real corrections, we take the transverse momentum of the $W$ boson in the reduced phase space, $\tilde{p}_{\mathrm{T}, W}$, as the factorization scale and the argument of $\alpha_{\mathrm{S}}$.

8 The use of different factorization scales, $\mu_{\mathrm{QCD}}=p_{\mathrm{T}}$ and $\mu_{\mathrm{QED}}=M_{W}$, is due to the fact that $\mu_{\mathrm{QCD}}$ and $\mu_{\mathrm{QED}}$ play a different role in our calculation. The dependence on $\mu_{\mathrm{QCD}}$ is due to the LO evolution of the PDFs and represents an effect of $\mathcal{O}\left(\alpha_{S} \ln \left(\mu_{\mathrm{QCD}} / \mu_{0}\right)\right)$, where $\mu_{0}$ is the scale at which the PDF evolution starts. This dependence would be compensated by NLO QCD contributions of $\mathcal{O}\left(\alpha_{S} \ln \left(p_{\mathrm{T}} / \mu_{\mathrm{QCD}}\right)\right)$ and, although QCD corrections are not included in our calculation, choosing $\mu_{\mathrm{QCD}}=p_{\mathrm{T}}$ we can absorb large NLO QCD logarithms of the scale $p_{\mathrm{T}}$ in the LO PDF evolution. In contrast, the $\mu_{\mathrm{QED}}$ dependence of our predictions is due to $\mathcal{O}\left(\alpha \ln \left(p_{\mathrm{T}} / \mu_{\mathrm{QED}}\right)\right)$ terms in the photon bremsstrahlung corrections. This dependence is not compensated by the PDF evolution since we use a PDF set that does not include QED effects, assuming that these effects are negligible. This approach makes sense only if the scale $\mu_{\mathrm{QED}}$ is chosen in such a way that the (potential) impact of QED effects on the PDFs is very small. In Ref. 37] it was shown that the QED corrections to the PDFs grow with $\mu_{\mathrm{QED}}$ but do not exceed one percent for $\mu_{\mathrm{QED}} \lesssim 100 \mathrm{GeV}$. This motivates our choice $\mu_{\mathrm{QED}}=M_{W}$ for the QED factorization scale.
} 
momentum distribution induced by variations of this parameter in the range $0.1 \leq$ $R_{\text {sep }} \leq 1.0$ does not exceed a few permille.

Our lowest-order (LO) predictions result from (32). The next-to-leading order predictions (NLO) include the LO+virtual contributions (173) and the real bremsstrahlung (89). We also study the relative importance of the IR-finite parts of the virtual $\left(\mathrm{NLO}_{\text {virt }}\right)$ and real $\left(\mathrm{NLO}_{\text {real }}\right)$ contributions. These IR-finite parts are constructed by subtracting the IR divergence (75) from the virtual corrections and adding it to the real ones. The next-to-leading-logarithmic (NLL) and next-tonext-leading-logarithmic (NNLL) predictions 9 are obtained adding to the LO the approximations (86) and (77) for the $\mathrm{NLO}_{\text {virt }}$ part and neglecting the $\mathrm{NLO}_{\text {real }}$ part of the corrections. As we will demonstrate, for the case of fully inclusive photon radiation neglecting this piece provides a good approximation of the complete calculation. The next-to-next-to-leading order predictions (NNLO) include the full NLO results plus the two-loop NLL corrections (87).

The LO transverse-momentum distributions for $p p \rightarrow W^{+} j$ and $p p \rightarrow W^{-} j$ at the LHC are shown in Fig. 5a. In Fig. 5b and Fig. 5 $\mathrm{c}$ we plot the relative size of the NLO, one-loop NLL, one-loop NNLL and NNLO corrections wrt. the LO predictions for $W^{+}$and $W^{-}$production, respectively. The behaviour of the relative corrections to $W^{+}$and $W^{-}$production is very similar. As expected, the importance of the NLO contribution increases significantly with $p_{\mathrm{T}}$ and leads to a negative correction ranging from $-15 \%$ at $p_{\mathrm{T}}=500 \mathrm{GeV}$ to $-43 \%$ at $p_{\mathrm{T}}=2 \mathrm{TeV}$. We also observe that the one-loop NLL and NNLL approximations are in good agreement (at the 1-2\% level) with the full NLO result for $p_{\mathrm{T}} \geq 100 \mathrm{GeV}$. The difference between NLO and NNLO curves is significant. The two-loop terms are positive and amount to $+3 \%$ at $p_{\mathrm{T}}=1 \mathrm{TeV}$ and $+9 \%$ at $p_{\mathrm{T}}=2 \mathrm{TeV}$. This shifts the relative corrections for $W^{+}$ production up to $-25 \%$ at $p_{\mathrm{T}}=1 \mathrm{TeV}$ and $-34 \%$ at $p_{\mathrm{T}}=2 \mathrm{TeV}$.

The IR-finite parts of the virtual $\left(\mathrm{NLO}_{\text {virt }}\right)$ and real $\left(\mathrm{NLO}_{\text {real }}\right)$ corrections to $W^{+}$production at the $\mathrm{LHC}$ are shown separately in Fig. 6a. Fig. 6b shows the relative size of the $\mathrm{NLO}_{\text {virt }}$ and $\mathrm{NLO}_{\text {real }}$ corrections wrt. the LO predictions. The $\mathrm{NLO}_{\text {virt }}$ contribution dominates the full NLO correction and amounts up to $-42 \%$ at $p_{\mathrm{T}}=2 \mathrm{TeV}$. The $\mathrm{NLO}_{\text {real }}$ part contributes with a smaller and nearly constant correction of about $-1 \%$ in the entire $p_{\mathrm{T}}$-range. This means that, for the case of fully inclusive photon radiation, the $\mathrm{NLO}_{\text {virt }}$ part represents a good approximation of the full NLO correction.

The high-energy behaviour of the $\mathrm{NLO}_{\text {virt }}$ part is described by the compact NLL and NNLL approximations presented in Sect. 4. The quality of these approximations is shown in Fig. 17. We observe that the NLL approximation works well differing from the exact $\mathrm{NLO}_{\text {virt }}$ result by less than $1 \%$ for $p_{\mathrm{T}} \geq 200 \mathrm{GeV}$. The quality of the NNLL approximation is of the order of one permille or better in the entire $p_{\mathrm{T}}$-range.

For less inclusive observables where a veto on hard photons is imposed, the $\mathrm{NLO}_{\text {real }}$ contribution can become important. Fig. 8 shows the relative $\mathrm{NLO}_{\text {real }}$ cor-

\footnotetext{
${ }^{9}$ For details concerning the treatment of angular-dependent logarithms at the NLL level we refer to Ref. [8].
} 


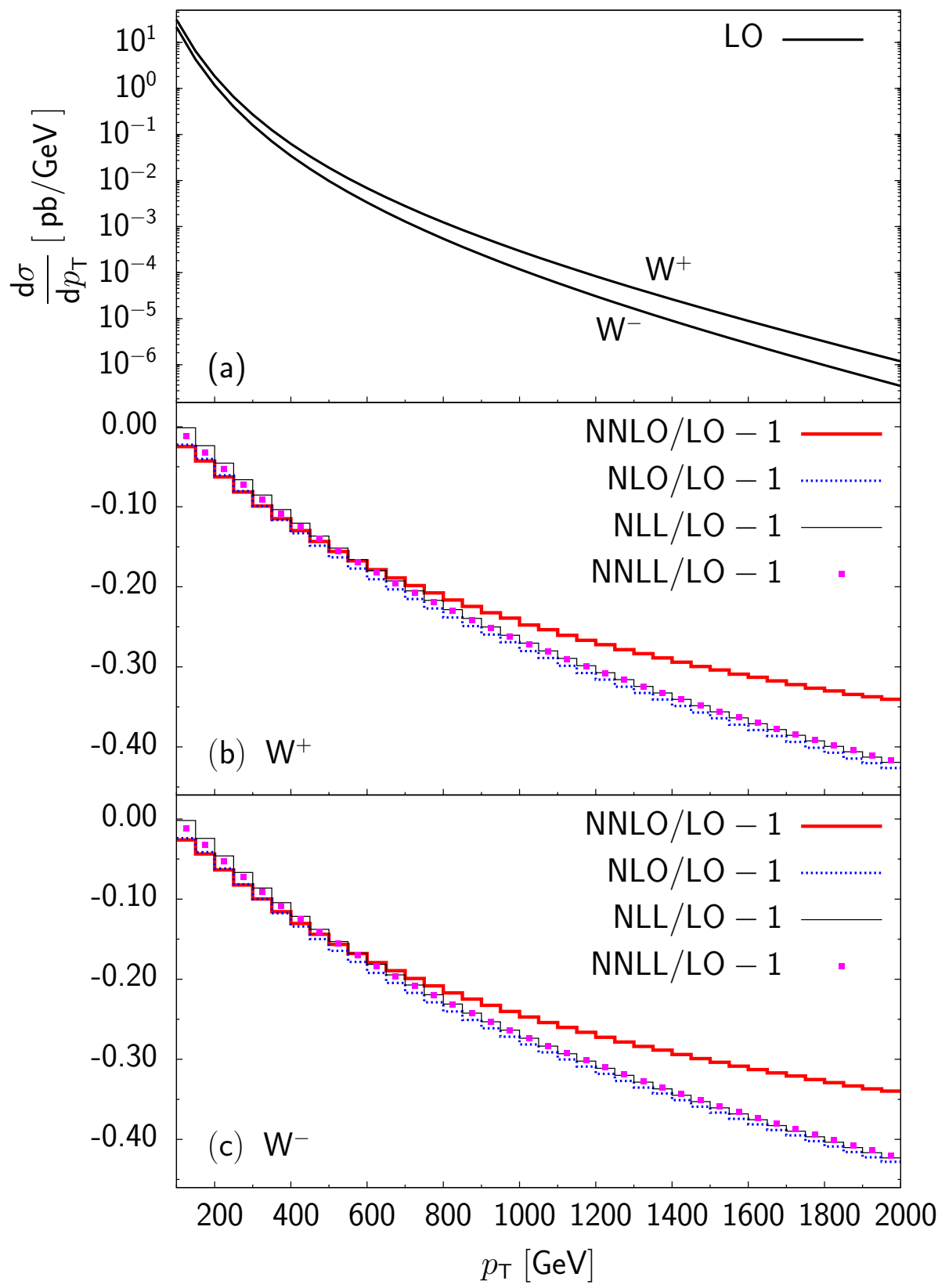

Figure 5: Transverse-momentum distribution for $W$-boson production at the LHC. (a) LO distribution for $p p \rightarrow W^{+} j$ and $p p \rightarrow W^{-} j$. (b) Relative NLO (dotted), NLL (thin solid), NNLL (squares) and NNLO (thick solid) electroweak correction wrt. the LO distribution for $p p \rightarrow W^{+} j$. (c) Relative NLO (dotted), NLL (thin solid), NNLL (squares) and NNLO (thick solid) electroweak correction wrt. the LO distribution for $p p \rightarrow W^{-} j$. 


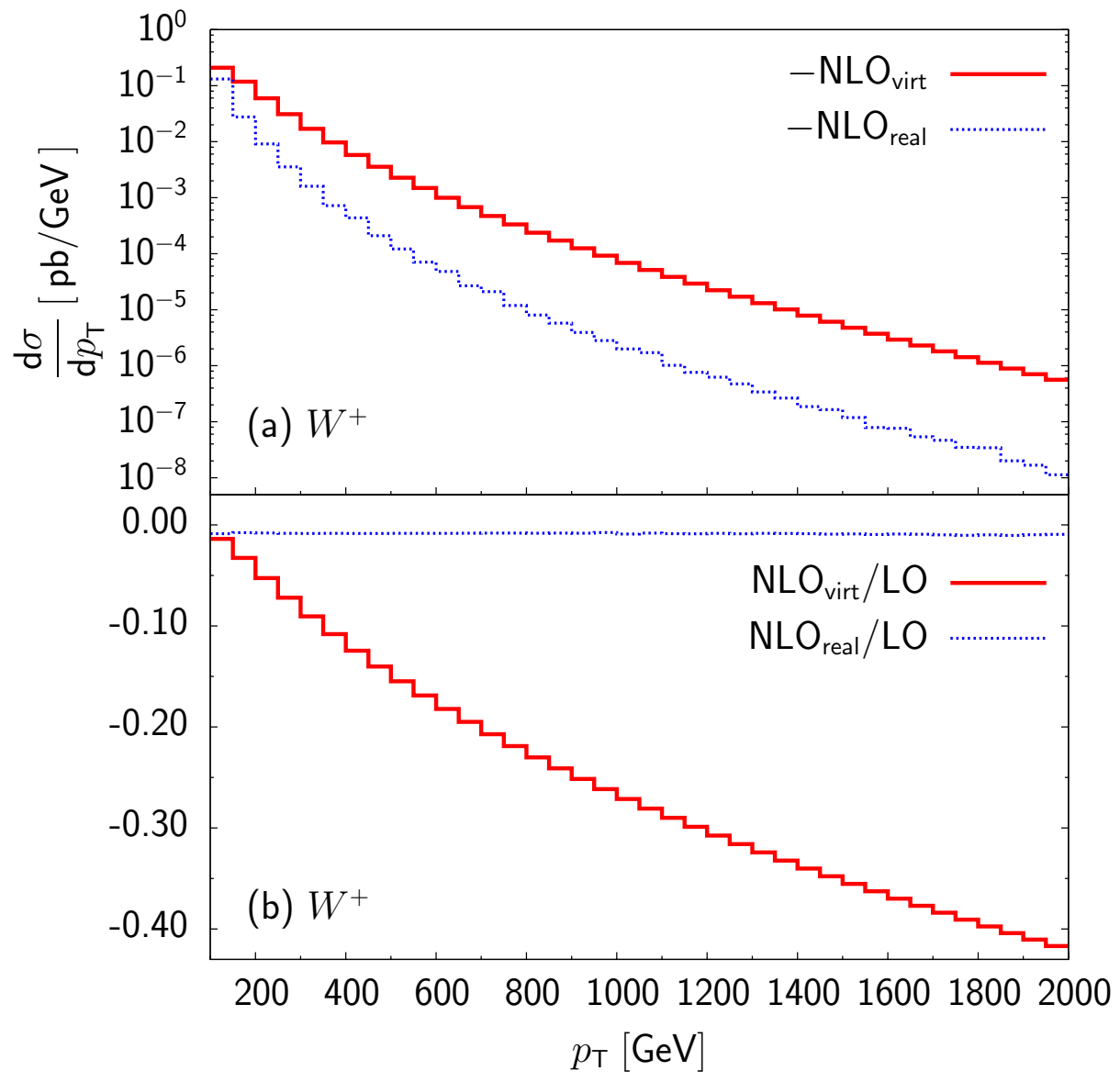

Figure 6: IR-finite parts of the virtual $\left(\mathrm{NLO}_{\text {virt }}\right)$ and real $\left(\mathrm{NLO}_{\text {real }}\right)$ contributions to the $p_{\mathrm{T}}$-distribution of $W$ bosons in the process $p p \rightarrow W^{+} j$ at $\sqrt{s}=14 \mathrm{TeV}$. 


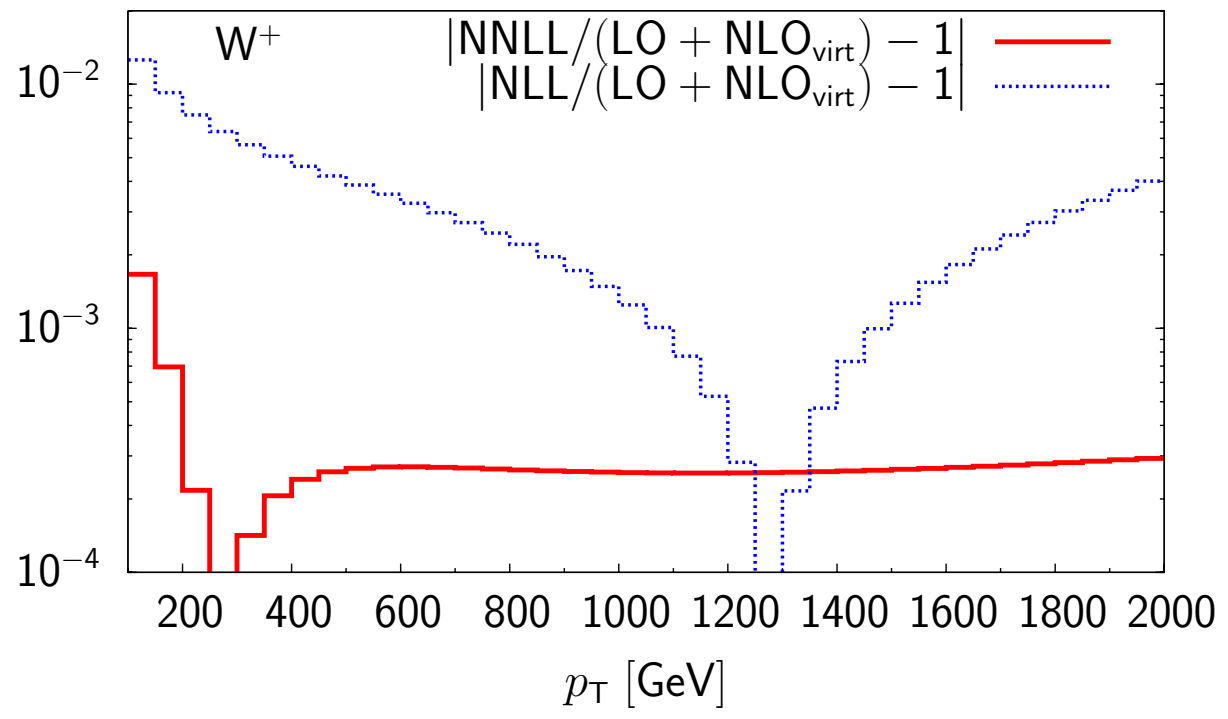

Figure 7: Relative precision of the high-energy approximations at one loop in the process $p p \rightarrow W^{+} j$ at $\sqrt{s}=14 \mathrm{TeV}$ as a function of $p_{\mathrm{T}}$ : NNLL (solid) and NLL (dashed) wrt. the IR-finite part of the exact one-loop result $\left(\mathrm{LO}+\mathrm{NLO}_{\text {virt }}\right)$.

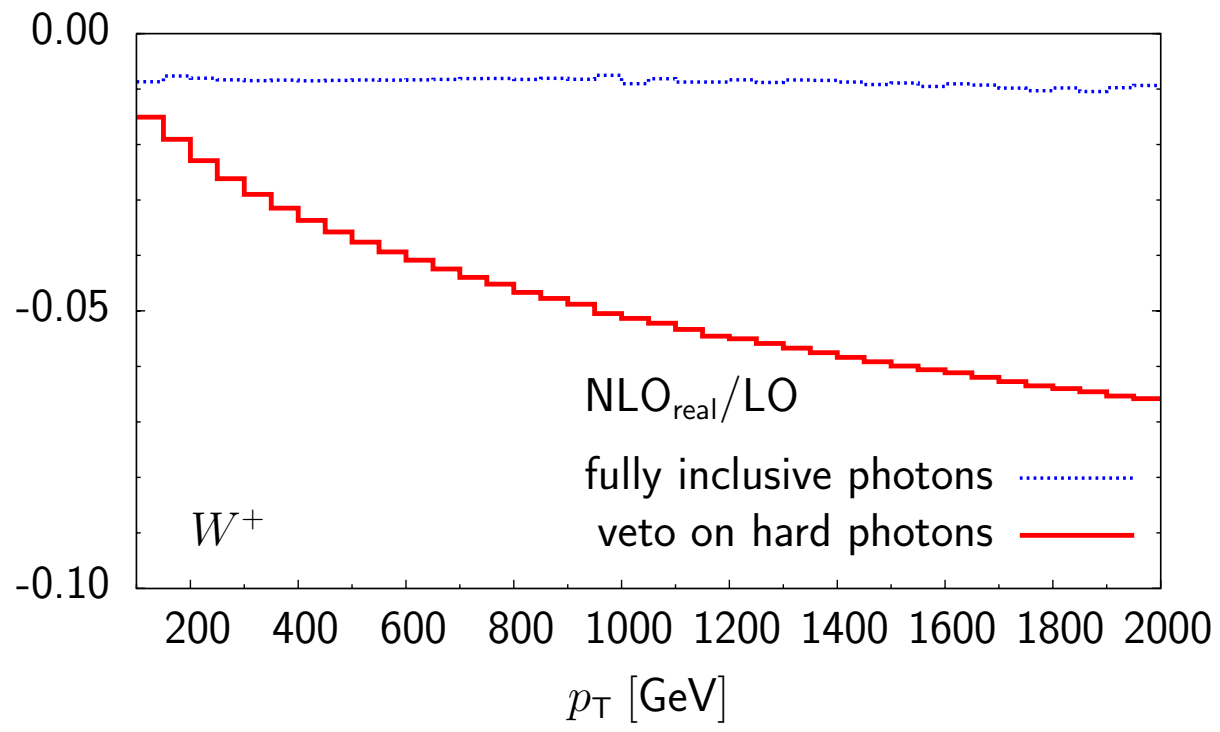

Figure 8: Relative size of the real correction ( $\left.\mathrm{NLO}_{\text {real }}\right)$ wrt. the LO for fully inclusive photon radiation and the case where visible photons are rejected, plotted as a function of $p_{\mathrm{T}}$ for the process $p p \rightarrow W^{+} j$ at $\sqrt{s}=14 \mathrm{TeV}$. 
rections for $W^{+}$production. We compare the fully inclusive photon radiation with the case where visible photons with $p_{\mathrm{T}, \gamma}>10 \mathrm{GeV}$ and $R(\gamma, j)>0.4$ are rejected. This veto leads to a significant enhancement of the (absolute size of the) $\mathrm{NLO}_{\text {real }}$ part, which can exceed $-5 \%$ for $p_{\mathrm{T}} \geq 1 \mathrm{TeV}$.

To underline the relevance of the large electroweak corrections for $W$ production at the LHC, in Fig. 9a and Fig. 9b we present the relative NLO and NNLO corrections to the $W^{+}$and $W^{-}$cross sections integrated over $p_{\mathrm{T}}$ starting from $p_{\mathrm{T}}=p_{\mathrm{T}}^{\text {cut }}$, as a function of $p_{\mathrm{T}}^{\text {cut }}$. This is compared with the statistical error, defined as $\Delta \sigma_{\text {stat }} / \sigma=1 / \sqrt{N}$ with $N=\mathcal{L} \times \mathrm{BR} \times \sigma_{\mathrm{LO}}$. The branching ratio $\mathrm{BR}=2 / 9$ accounts for the full efficiency of $W$-detection in the $e \overline{\nu_{e}}$ and $\mu \overline{\nu_{\mu}}$ modes (for this estimate we ignore experimental efficiencies and cuts) and we assume a total integrated luminosity $\mathcal{L}=300 \mathrm{fb}^{-1}$ for the LHC [38]. It is clear that the size of the NLO corrections is much bigger than the statistical error. Indeed, already for $\mathcal{L}=3 \mathrm{fb}^{-1}$ and $p_{\mathrm{T}} \lesssim 800 \mathrm{GeV}$ they correspond to a two standard deviation effect. Also the difference between the NNLO and NLO corrections, due to two-loop logarithmic effects, is significant. In terms of the estimated statistical error, these two-loop contributions amount to 1-3 standard deviations for $p_{\mathrm{T}}$ of $\mathcal{O}(1 \mathrm{TeV})$.

Ratios of $p_{\mathrm{T}}$-distributions for $W^{+}, W^{-}, Z$ bosons [9] and photons [10], in contrast to the distributions themselves are expected to be relatively insensitive to QCD corrections and theoretical uncertainties associated with $\alpha_{\mathrm{S}}$ and PDFs. These ratios lead to important experimental tests of $W$ and $Z$ couplings in the high-energy region. For $W^{+}$and $W^{-}$the ratio is presented in Fig. 10a. The LO value increases from 1.5 at $p_{\mathrm{T}}=100 \mathrm{GeV}$ to 3.4 at $p_{\mathrm{T}}=2 \mathrm{TeV}$. As already observed, the (relative) electroweak corrections to the $W^{+}$- and $W^{-}$-boson $p_{\mathrm{T}}$-distributions are almost identical. In consequence, the LO, NLO and NNLO curves in Fig. 10a overlap. In contrast, the impact of the electroweak corrections on the $W^{+} / \gamma$ ratio (Fig. 10b) at the LHC is clearly visible. The LO prediction, ranging from 1.4 to 2.5 , receives a negative NLO correction that grows with $p_{\mathrm{T}}$ and amounts to -0.5 for $p_{\mathrm{T}}=1 \mathrm{TeV}$. At $p_{\mathrm{T}}=2 \mathrm{TeV}$ the difference between the NNLO and NLO curves is about 0.2.

The ratios of $p_{\mathrm{T}^{-}}$-distributions for $W^{+} / Z$ and $W^{-} / Z$ are shown in Fig. 11a and Fig. 11b, respectively. For the $W^{+} / Z$ ratio the LO prediction ranges from 1.5 to 2 . For $p_{\mathrm{T}} \geq 1 \mathrm{TeV}$ it is reduced by 0.09 to 0.18 by the NLO electroweak corrections. The logarithmic two-loop corrections are small. A qualitatively similar behaviour is observed for the $W^{-} / Z$ ratio.

The results of a similar analysis for $W^{+}$production at the Tevatron $(\sqrt{s}=2 \mathrm{TeV})$ are shown in Figs. 12 15 (the $p_{\mathrm{T}}$-distributions for $W^{+}$and $W^{-}$production are obviously identical). The LO $p_{\mathrm{T}^{-}}$-distribution is shown in Fig. 12a, the relative NLO, NLL, NNLL and NNLO corrections in Fig. 12 b. The NLO corrections grow with $p_{\mathrm{T}}$ and reach $-11 \%$ at $p_{\mathrm{T}}=400 \mathrm{GeV}$. The one-loop NNLL and NLL approximations describe the exact NLO results with $1 \%$ and $3 \%$ precision, respectively. The dominant two-loop effects have little impact on the size of the corrections.

The quality of the high-energy approximations wrt. the IR-finite part of the virtual corrections $\left(\mathrm{NLO}_{\text {virt }}\right)$ is shown in Fig. 13, In the $p_{\mathrm{T}}$-range under considera- 


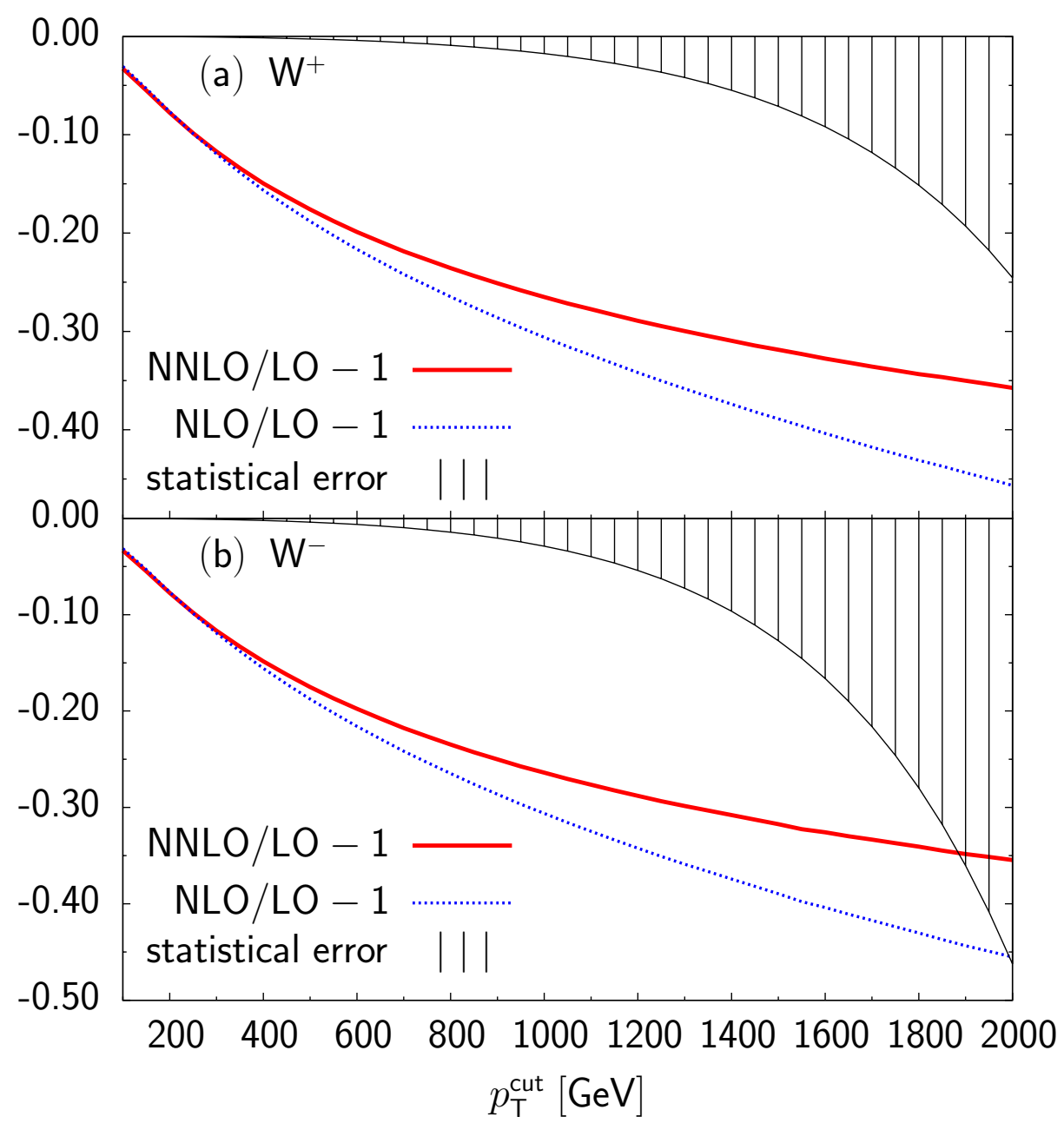

Figure 9: Relative NLO (dotted) and NNLO (solid) electroweak corrections wrt. the LO and statistical error (shaded area) for the unpolarized integrated cross section for (a) $p p \rightarrow W^{+} j$ at $\sqrt{s}=14 \mathrm{TeV}$ and (b) $p p \rightarrow W^{-} j$ at $\sqrt{s}=14 \mathrm{TeV}$ as a function of $p_{\mathrm{T}}^{\text {cut }}(W)$. 


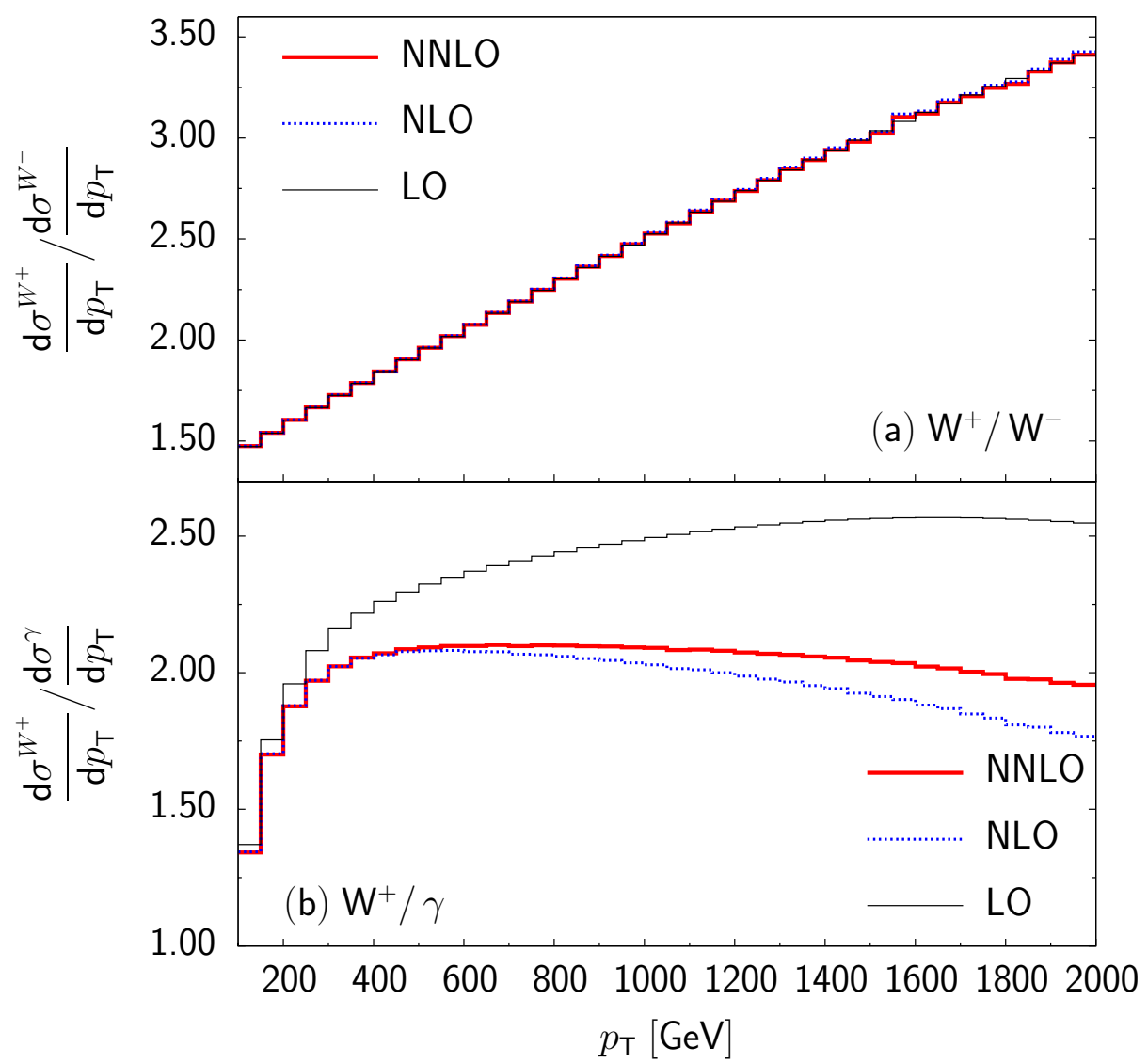

Figure 10: Ratio of the $p_{\mathrm{T}^{-}}$distributions for the processes (a) $p p \rightarrow W^{+} j$ and $p p \rightarrow W^{-} j$ and (b) $p p \rightarrow W^{+} j$ and $p p \rightarrow \gamma j$ at $\sqrt{s}=14 \mathrm{TeV}$ : LO (thin solid), NLO(dotted) and NNLO (thick solid) predictions. 


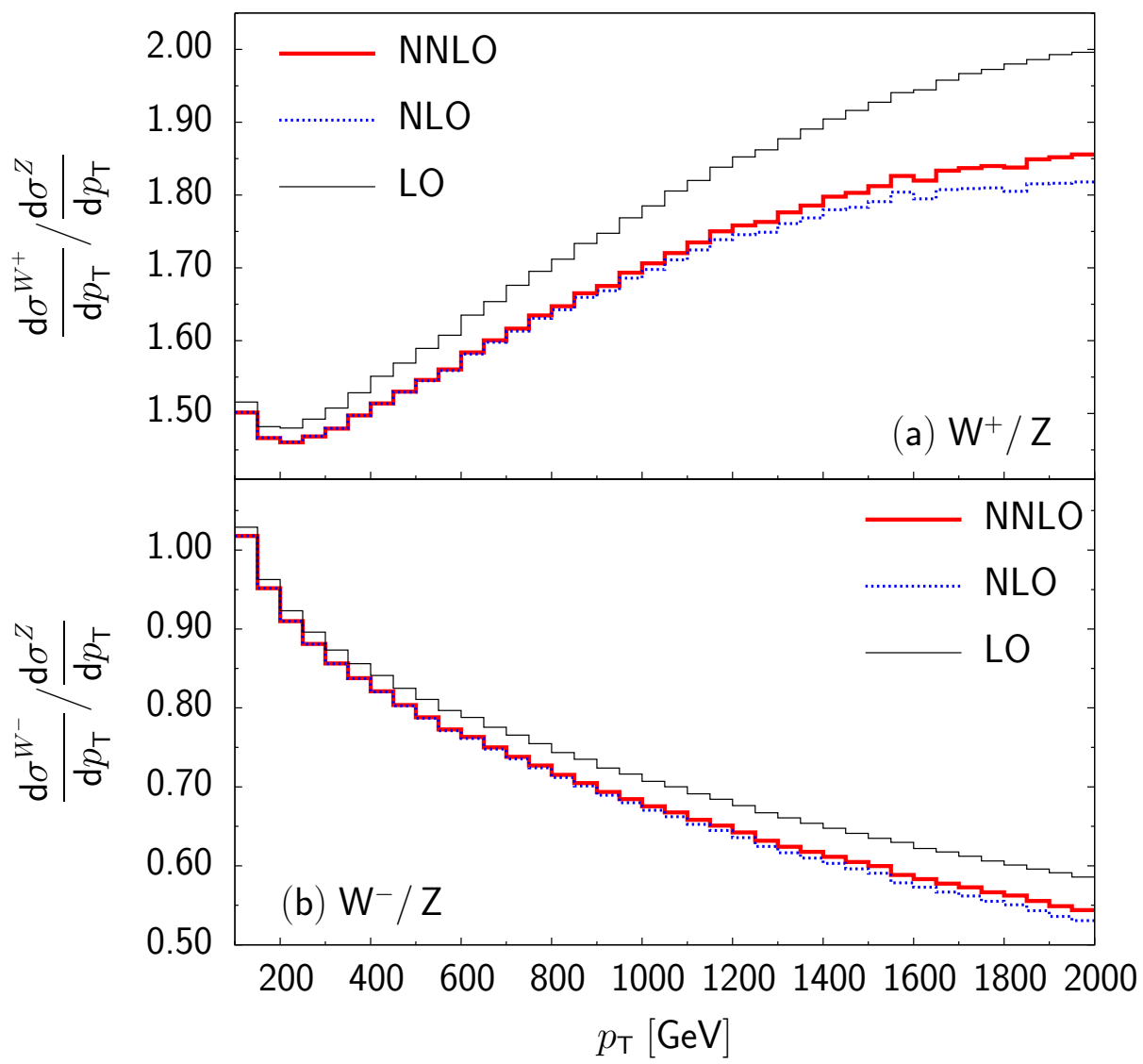

Figure 11: Ratio of the $p_{\mathrm{T}}$-distributions for the processes (a) $p p \rightarrow W^{+} j$ and $p p \rightarrow Z j$ and (b) $p p \rightarrow W^{-} j$ and $p p \rightarrow Z j$ at $\sqrt{s}=14 \mathrm{TeV}: \mathrm{LO}$ (thin solid), NLO(dotted) and NNLO (thick solid) predictions. 
tion both approximations are less precise than at the LHC, nevertheless the NNLL approximation in sufficient for all practical purposes.

In Fig. 14 the relative NLO and NNLO corrections to the integrated cross section for $p_{\mathrm{T}} \geq p_{\mathrm{T}}^{\text {cut }}$ are compared with the expected statistical error for an integrated luminosity $\mathcal{L}=7 \mathrm{fb}^{-1}$ [39]. The size of the NLO electroweak corrections is above the statistical error for a significant range of $p_{\mathrm{T}}$-values. Therefore they should be included in the analysis when considering precision measurements. In contrast, the impact of the dominant two-loop corrections is negligible.

The effect of the electroweak corrections on the ratios of $p_{\mathrm{T}}$-distributions for $W / Z$ and for $W / \gamma$ is shown in Fig. [15a and Fig. 15b, respectively.

\section{Summary}

In this work the electroweak corrections to large transverse momentum production of $W$ bosons at the hadron colliders Tevatron and LHC were evaluated. The contributions from real and virtual photons cannot be separated in a gauge-invariant manner from purely weak corrections and were thus included in our analysis. Soft and collinear singularities were regulated by introducing a small quark mass and a small photon mass and, alternatively, by using dimensional regularization. The real photon radiation was evaluated using the dipole subtraction formalism. The agreement between the results derived in the two regularization schemes has been an important cross check of the calculation. Numerous additional tests were performed to ensure the correctness of the result.

At the Tevatron, $p_{\mathrm{T}}$-values up to around $300 \mathrm{GeV}$ can be reached with reasonable event rates. In this region the $\mathcal{O}(\alpha)$ electroweak corrections reach up to $-10 \%$ and are thus of relevance for precision measurements. Two-loop electroweak corrections are negligible at the Tevatron. With $p_{\mathrm{T}}$ below $400 \mathrm{GeV}$ the relative rates for $W, Z$ and $\gamma$ production are hardly affected by electroweak corrections.

In contrast, for transverse momenta in the TeV region accessible at the LHC, electroweak corrections play an important role. The $\mathcal{O}(\alpha)$ corrections lead to a reduction of the cross section by about $-15 \%$ at transverse momenta of $500 \mathrm{GeV}$ and reach more than $-40 \%$ at $2 \mathrm{TeV}$. The logarithmically dominant terms were extracted from the exact expression of the virtual corrections and agreement was found with the predictions based on the process-independent analysis of electroweak Sudakov logarithms. If no cuts on real photons are applied, the contribution of the real photon emission is numerically small (about 1\%) and almost independent of $p_{\mathrm{T}}$. Numerically the NLL and NNLL approximations give a good description of the full $\mathcal{O}(\alpha)$ result with an accuracy of about $1-2 \%$. Considering the large event rate at the LHC, leading to a fairly good statistical precision even at transverse momenta up to $2 \mathrm{TeV}$, we evaluated also the dominant (NLL) two-loop terms. In the high- $p_{\mathrm{T}}$ region, these two-loop logarithmic effects increase the cross section by $5-10 \%$ and thus become of importance in precision studies. We also studied the relative rates 
for $W^{+}, W^{-}, Z$ and $\gamma$ production, which are expected to be stable with respect to QCD effects. The electroweak corrections cancel almost completely in the $W^{+} / W^{-}$ ratio. In contrast, their impact on the $W^{+} / Z$ and the $W^{+} / \gamma$ ratios is significant and leads to a shift of $\mathcal{O}(10 \%)$ for $p_{\mathrm{T}} \geq 1 \mathrm{TeV}$.

\section{Acknowledgements}

We would like to thank S. Dittmaier, B. Jäger and P. Uwer for helpful discussions. This work was supported in part by BMBF Grant No. 05HT4VKA/3, the Sonderforschungsbereich Transregio 9 and the DFG Graduiertenkolleg "Hochenergiephysik und Teilchenastrophysik". 


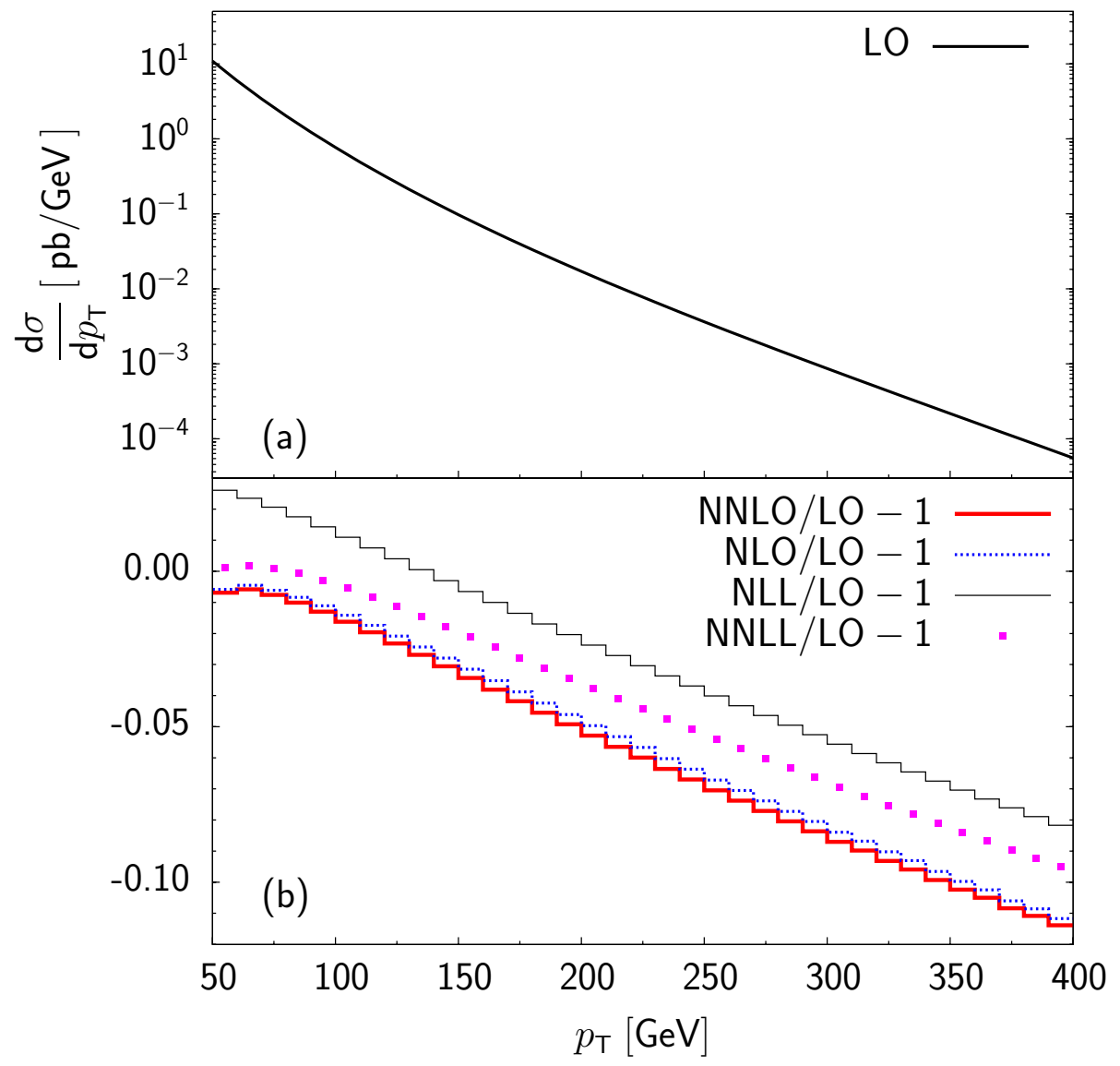

Figure 12: Transverse-momentum distribution for $W$-boson production at the Tevatron. (a) LO distribution for $p \bar{p} \rightarrow W^{+(-)} j$ (solid). (b) Relative NLO (dotted), NLL (thin solid), NNLL (squares) and NNLO (thick solid) electroweak correction wrt. the LO distribution for $p p \rightarrow W^{+(-)} j$. 


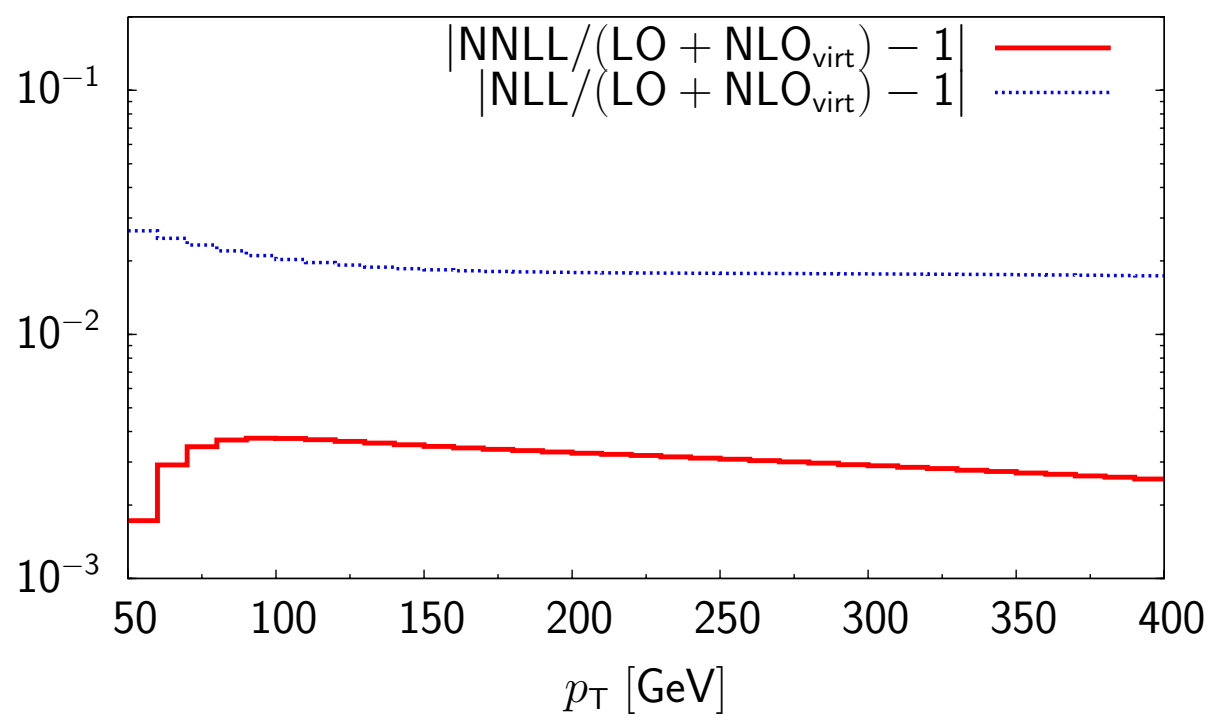

Figure 13: Relative precision of the high-energy approximations at one loop in the process $p \bar{p} \rightarrow W^{+(-)} j$ at $\sqrt{s}=2 \mathrm{TeV}$ as a function of $p_{\mathrm{T}}$ : NNLL (solid) and NLL (dashed) wrt. the IR-finite part of the exact one-loop result $\left(\mathrm{LO}+\mathrm{NLO}_{\text {virt }}\right)$.

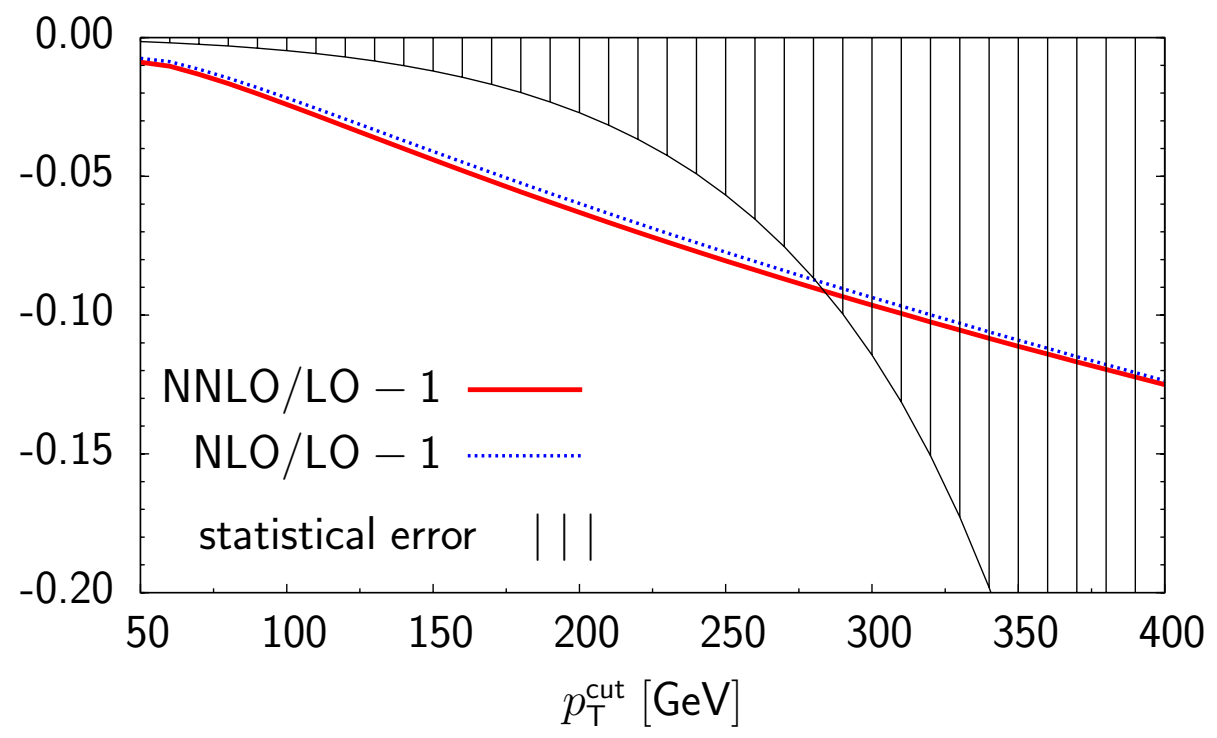

Figure 14: Relative NLO (dotted) and NNLO (solid) electroweak corrections wrt. the LO and statistical error (shaded area) for the unpolarized integrated cross section for $p \bar{p} \rightarrow W^{+(-)} j$ at $\sqrt{s}=2 \mathrm{TeV}$ as a function of $p_{\mathrm{T}}^{\text {cut }}(W)$. 


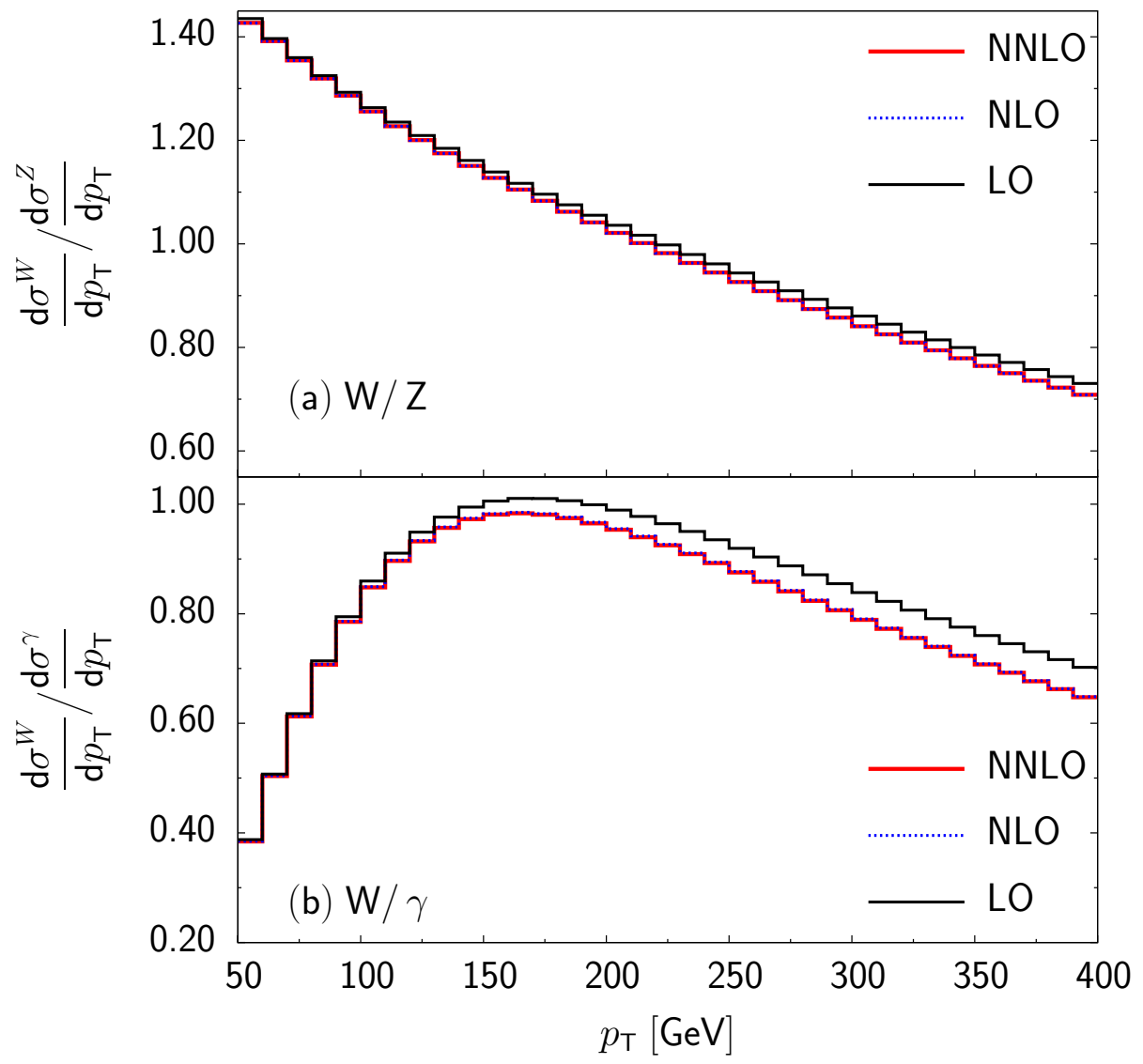

Figure 15: Ratio of the $p_{\mathrm{T}}$-distributions (a) for the processes $p \bar{p} \rightarrow W^{+(-)} j$ and $p \bar{p} \rightarrow Z j$ and (b) for the processes $p \bar{p} \rightarrow W^{+(-)} j$ and $p \bar{p} \rightarrow \gamma j$ at $\sqrt{s}=2 \mathrm{TeV}:$ LO (thin solid), NLO(dotted) and NNLO (thick solid) predictions. 


\section{A Recombination and exclusive $W j$ cross section}

As discussed in Sect. 2.2, the recombination prescription that we use to regularize photon-quark final-state collinear singularities implies a different treatment of finalstate quarks and gluons. While for final-state gluons we apply a cut on $p_{\mathrm{T}, g}$ within the entire phase space, for final-state quarks the recombination effectively removes the cut on $p_{\mathrm{T}, q}$ inside the collinear cone $R(q, \gamma)<R_{\text {sep }}$. As a consequence the recombined $g q^{\prime} \rightarrow W^{\sigma} q \gamma$ cross section (18) has a logarithmic dependence on the cut-off parameter $R_{\text {sep }}$. In order to quantify this $R_{\text {sep-dependence, let us consider }}$ the contribution of real photon radiation inside the recombination cone. To this end, assuming that the cone is sufficiently small $\left(R_{\text {sep }} \ll 1\right)$, we adopt a collinear approximation

$$
\int_{R(q, \gamma)<R_{\mathrm{sep}}} \mathrm{d} \hat{\sigma}^{g q^{\prime} \rightarrow W^{\sigma} q \gamma}=\hat{\sigma}^{g q^{\prime} \rightarrow W^{\sigma} q} \int_{0}^{1} \mathrm{~d} z F_{q \gamma}(z),
$$

where $10 z=p_{\mathrm{T}, \gamma} /\left(p_{\mathrm{T}, q}+p_{\mathrm{T}, \gamma}\right)=1-p_{\mathrm{T}, q} / p_{\mathrm{T}, W}$ is the photon momentum fraction and [18]

$$
\begin{aligned}
F_{q \gamma}(z) & =\frac{\alpha Q_{q}^{2}}{2 \pi} P_{q \gamma}(z, \varepsilon) \frac{\left(4 \pi \mu^{2}\right)^{\varepsilon}}{\Gamma(1-\varepsilon)} \int_{0}^{k_{\perp, \max }^{2}} \frac{\mathrm{d} k_{\perp}^{2}}{\left(k_{\perp}^{2}\right)^{1+\varepsilon}} \\
& =-\frac{\alpha Q_{q}^{2}}{2 \pi} P_{q \gamma}(z) \frac{(4 \pi)^{\varepsilon}}{\varepsilon \Gamma(1-\varepsilon)}+\bar{F}_{q \gamma}\left(z, \mu^{2}\right)
\end{aligned}
$$

with

$$
\bar{F}_{q \gamma}\left(z, \mu^{2}\right)=-\frac{\alpha Q_{q}^{2}}{2 \pi}\left[P_{q \gamma}(z) \ln \left(\frac{\mu^{2}}{k_{\perp, \max }^{2}}\right)-z\right] .
$$

Here $P_{q \gamma}(z, \varepsilon)=P_{q \gamma}(z)-\varepsilon z$ with $P_{q \gamma}(z)=\left[1+(1-z)^{2}\right] / z$ is the $q \rightarrow \gamma$ splitting function in $4-2 \varepsilon$ dimensions, $k_{\perp}$ is the photon tranverse momentum wrt. the photonquark system, and $k_{\perp, \max }=z(1-z) R_{\mathrm{sep}} p_{\mathrm{T}, W}$. The $1 / \varepsilon$ collinear singularity resulting from inclusive photon radiation, i.e. integrating over the complete energy spectrum $0 \leq z \leq 1$, cancels against the virtual corrections.

The $R_{\mathrm{sep}}$-dependence of the recombined cross section (18) is due to the fact that, inside the recombination cone quarks with $p_{\mathrm{T}, q}<p_{\mathrm{T}, j}^{\min }$ (or equivalently photons with $\left.z>1-p_{\mathrm{T}, j}^{\min } / p_{\mathrm{T}, W}\right)$ are not rejected. Thus the variation of $\hat{\sigma}_{\text {rec. }}$ induced by a rescaling $R_{\text {sep }} \rightarrow \xi_{\text {sep }} R_{\text {sep }}$ amounts to

$$
\frac{\Delta \hat{\sigma}_{\text {rec. }}^{g q^{\prime} \rightarrow W^{\sigma} q \gamma}}{\hat{\sigma}^{g q^{\prime} \rightarrow W^{\sigma} q}}=\frac{\alpha Q_{q}^{2}}{2 \pi} \ln \xi_{\text {sep }}^{2} \int_{z_{\min }}^{1} \mathrm{~d} z P_{q \gamma}(z) \quad \text { with } \quad z_{\min }=1-p_{\mathrm{T}, j}^{\min } / p_{\mathrm{T}, W} .
$$

For relatively small transverse momenta $\left(p_{\mathrm{T}, W} \simeq 2 p_{\mathrm{T}, j}^{\min }\right)$ a rescaling of $R_{\text {sep }}$ by a factor $\xi_{\text {sep }}=10$ shifts the $g q^{\prime} \rightarrow W^{\sigma} q(\gamma)$ cross section by less than $2(0.5)$ permille for up-

\footnotetext{
${ }^{10}$ Here we assume lowest-order kinematics, i.e. $p_{\mathrm{T}, q}+p_{\mathrm{T}, \gamma}=p_{\mathrm{T}, W}$ in the collinear region.
} 
(down-) type quarks. Moreover it is obvious that at high $p_{\mathrm{T}, W}$, where $z_{\min } \rightarrow 1$, this effect tends to disappear.

Let us now compare the recombination procedure with a realistic definition of exclusive $p p \rightarrow W j$ production, where final-state quarks $(a=q)$ and gluons $(a=g)$ are subject to the same cut $p_{\mathrm{T}, a}>p_{\mathrm{T}, j}^{\min }$ within the entire phase space (including collinear quark-photon configurations). Since the recombination procedure does not affect final-state gluons, only channels involving final-state quarks need to be considered. The difference between the recombined $g q^{\prime} \rightarrow W^{\sigma} q \gamma$ cross section (18) and the exclusive cross section (19) corresponds to the contribution of hard collinear photons with $R(q, \gamma)<R_{\text {sep }}$ and $z_{\text {min }} \leq z \leq 1$. This collinear hard-photon radiation can be described by means of quark fragmentation functions [43, 44, 45, 46, 47, 48] as

$$
\Delta \hat{\sigma}_{\text {excl. }}=\hat{\sigma}_{\text {rec. }}^{g q^{\prime} \rightarrow W^{\sigma} q \gamma}-\hat{\sigma}_{\text {excl. }}^{g q^{\prime} \rightarrow W^{\sigma} q \gamma}=\hat{\sigma}^{g q^{\prime} \rightarrow W^{\sigma} q} \int_{z_{\min }}^{1} \mathrm{~d} z \mathcal{D}_{q \gamma}(z)
$$

Here the effective quark fragmentation function $\mathcal{D}_{q \gamma}(z)=F_{q \gamma}(z)+D_{q \gamma}(z)$ consists of the perturbative contribution $F_{q \gamma}$ and the bare fragmentation function $D_{q \gamma}$. The collinear singularity resulting from the perturbative contribution is factorized into the bare fragmentation function at the scale $\mu$, such that in the $\overline{\mathrm{MS}}$ scheme [43]

$$
\mathcal{D}_{q \gamma}(z)=\bar{F}_{q \gamma}\left(z, \mu^{2}\right)+\bar{D}_{q \gamma}\left(z, \mu^{2}\right)
$$

and the renormalized fragmentation function $\bar{D}_{q \gamma}$ can be extracted from experimental measurements. Using the parametrization [44, 48]

$$
\bar{D}_{q \gamma}\left(z, \mu_{0}^{2}\right)=\frac{\alpha Q_{q}^{2}}{2 \pi}\left[-P_{q \gamma}(z) \ln (1-z)^{2}-13.26\right]
$$

obtained by the ALEPH collaboration at $\mu_{0}=0.14 \mathrm{GeV}$, we arrive at

$$
\mathcal{D}_{q \gamma}(z)=\frac{\alpha Q_{q}^{2}}{2 \pi}\left[P_{q \gamma}(z) \ln \left(\frac{z R_{\mathrm{sep}} p_{\mathrm{T}, W}}{\mu_{0}}\right)^{2}+z-13.26\right]
$$

With this expression we derive a conservative upper bound for $\Delta \hat{\sigma}_{\text {excl. }}$. To this end we consider $Q_{q}=2 / 3, R_{\mathrm{sep}} \simeq 1$, and a wide range of transverse momenta, $2 p_{\mathrm{T}, j}^{\min } \leq p_{\mathrm{T}, W} \leq 2 \mathrm{TeV}$. With these parameters we obtain

$$
\frac{\Delta \hat{\sigma}_{\text {excl. }}}{\hat{\sigma}} \lesssim 2 \times 10^{-3}
$$

We conclude that, for $R_{\text {sep }} \lesssim \mathcal{O}(1)$, the recombined cross section has a negligible dependence on the recombination parameter $R_{\text {sep }}$ and provides a fairly precise description of exclusive $p p \rightarrow W j$ production at high transverse momentum. 


\section{B Standard matrix elements}

The algebraic expressions involving external momenta, Dirac matrices, spinors and

gauge-boson polarization vectors have been reduced to a set of 10 standard matrix elements

$$
\mathcal{S}_{i}=\bar{v}\left(p_{\bar{q}}\right) \mathcal{S}_{i}^{\mu \nu} \omega_{\mathrm{L}} u\left(p_{q^{\prime}}\right) \varepsilon_{\mu}^{*}\left(p_{W}\right) \varepsilon_{\nu}^{*}\left(p_{g}\right)
$$

with

$$
\begin{aligned}
& \mathcal{S}_{1}^{\mu \nu}=\gamma^{\mu}\left(\not p_{W}-\not p_{\bar{q}}\right) \gamma^{\nu}, \\
& \mathcal{S}_{2}^{\mu \nu}=\left(\not \not_{W}-\not p_{g}\right) g^{\mu \nu}, \\
& \mathcal{S}_{3}^{\mu \nu}=\gamma^{\mu} p_{W}^{\nu}, \\
& \mathcal{S}_{4}^{\mu \nu}=-\gamma^{\nu} p_{g}^{\mu}, \\
& \mathcal{S}_{5}^{\mu \nu}=\gamma^{\mu} p_{q^{\prime}}^{\nu}, \\
& \mathcal{S}_{6}^{\mu \nu}=-\gamma^{\nu} p_{\bar{q}}^{\mu}, \\
& \mathcal{S}_{7}^{\mu \nu}=\left(\not p_{W}-\not p_{g}\right) p_{g}^{\mu} p_{W}^{\nu}, \\
& \mathcal{S}_{8}^{\mu \nu}=\left(\not p_{W}-\not p_{g}\right) p_{\bar{q}}^{\mu} p_{q^{\prime}}^{\nu}, \\
& \mathcal{S}_{9}^{\mu \nu}=\left(\not p_{W}-\not p_{g}\right) p_{g}^{\mu} p_{q^{\prime}}^{\nu}, \\
& \mathcal{S}_{10}^{\mu \nu}=\left(\not \not_{W}-\not p_{g}\right) p_{\bar{q}}^{\mu} p_{W}^{\nu} .
\end{aligned}
$$

These algebraic expressions correspond to the massless subset of the standard matrix elements of Ref. [25].

\section{Scalar loop integrals}

In this appendix we list the scalar loop integrals $J_{j}\left(M_{V}^{2}\right)$ that contribute to (48). The symbols $J_{j}$ are chosen in analogy with Ref. [9]. For convenience, to denote constant terms we define

$$
J_{0}\left(M_{V}^{2}\right)=1 .
$$

For the scalar integrals $A_{0}, B_{0}, C_{0}$ and $D_{0}$ we adopt the notation of FEYNCALC [40]. However, we choose their normalization according to Ref. [25], i.e. we include the factor $(2 \pi \mu)^{4-D}$ which is omitted in the conventions of FEYNCALC.

The UV-divergent one- and two-point functions are denoted as

$$
\begin{aligned}
J_{1 a}\left(M_{V}^{2}\right) & =B_{0}\left(m^{2} ; M_{V}^{2}, m^{2}\right), \\
J_{1 b}\left(M_{V}^{2}\right) & =B_{0}\left(m^{2} ; M_{W}^{2}, m^{2}\right)=J_{1 a}\left(M_{W}^{2}\right), \\
J_{2}\left(M_{V}^{2}\right) & =B_{0}\left(p_{W}^{2} ; m^{2}, m^{2}\right), \\
J_{3}\left(M_{V}^{2}\right) & =B_{0}\left(p_{W}^{2} ; M_{W}^{2}, M_{V}^{2}\right), \\
J_{4}\left(M_{V}^{2}\right) & =B_{0}\left(\hat{s} ; m^{2}, m^{2}\right), \\
J_{5 a}\left(M_{V}^{2}\right) & =B_{0}\left(\hat{u} ; M_{V}^{2}, m^{2}\right),
\end{aligned}
$$




$$
\begin{aligned}
& J_{5 b}\left(M_{V}^{2}\right)=B_{0}\left(\hat{u} ; M_{W}^{2}, m^{2}\right)=J_{5 a}\left(M_{W}^{2}\right), \\
& J_{6 a}\left(M_{V}^{2}\right)=B_{0}\left(\hat{t} ; M_{V}^{2}, m^{2}\right), \\
& J_{6 b}\left(M_{V}^{2}\right)=B_{0}\left(\hat{t} ; M_{W}^{2}, m^{2}\right)=J_{6 a}\left(M_{W}^{2}\right) .
\end{aligned}
$$

The remaining loop integrals are free from UV singularities. The following threepoint functions are finite if $M_{V}$ and the $W$-boson transverse momentum are nonvanishing:

$$
\begin{aligned}
J_{7}\left(M_{V}^{2}\right) & =C_{0}\left(\hat{s}, m^{2}, m^{2} ; m^{2}, m^{2}, M_{V}^{2}\right), \\
J_{8}\left(M_{V}^{2}\right) & =C_{0}\left(\hat{u}, p_{W}^{2}, m^{2} ; M_{V}^{2}, m^{2}, m^{2}\right), \\
J_{9 a}\left(M_{V}^{2}\right) & =C_{0}\left(\hat{u}, p_{W}^{2}, m^{2} ; m^{2}, M_{W}^{2}, M_{V}^{2}\right), \\
J_{9 b}\left(M_{V}^{2}\right) & =C_{0}\left(\hat{u}, p_{W}^{2}, m^{2} ; m^{2}, M_{V}^{2}, M_{W}^{2}\right)=\left.J_{9 a}\left(M_{V}^{2}\right)\right|_{M_{V}^{2} \leftrightarrow M_{W}^{2}}, \\
J_{10}\left(M_{V}^{2}\right) & =C_{0}\left(\hat{t}, p_{W}^{2}, m^{2} ; M_{V}^{2}, m^{2}, m^{2}\right)=\left.J_{8}\left(M_{V}^{2}\right)\right|_{\hat{t} \leftrightarrow \hat{u}}, \\
J_{11 a}\left(M_{V}^{2}\right) & =C_{0}\left(\hat{t}, p_{W}^{2}, m^{2} ; m^{2}, M_{W}^{2}, M_{V}^{2}\right)=\left.J_{9 a}\left(M_{V}^{2}\right)\right|_{\hat{t} \leftrightarrow \hat{u}}, \\
J_{11 b}\left(M_{V}^{2}\right) & =C_{0}\left(\hat{t}, p_{W}^{2}, m^{2} ; m^{2}, M_{V}^{2}, M_{W}^{2}\right)=\left.J_{9 b}\left(M_{V}^{2}\right)\right|_{\hat{t} \leftrightarrow \hat{u}},
\end{aligned}
$$

In addition, the box diagrams b1-b3 in Fig. 2, provide the following combinations of three- and four-point functions

$$
\begin{aligned}
J_{12}\left(M_{V}^{2}\right)= & D_{0}\left(m^{2}, 0, p_{W}^{2}, m^{2}, \hat{u}, \hat{s} ; M_{V}^{2}, m^{2}, m^{2}, m^{2}\right)-\frac{1}{\hat{s} \hat{u}+(\hat{t}+\hat{u}) M_{V}^{2}} \\
& \times\left[\left(\hat{u}-p_{W}^{2}\right) C_{0}\left(\hat{u}, p_{W}^{2}, m^{2} ; M_{V}^{2}, m^{2}, m^{2}\right)+\hat{u} C_{0}\left(\hat{u}, 0, m^{2} ; M_{V}^{2}, m^{2}, m^{2}\right)\right. \\
& \left.+\left(\hat{s}-p_{W}^{2}\right) C_{0}\left(\hat{s}, p_{W}^{2}, 0 ; m^{2}, m^{2}, m^{2}\right)\right], \\
J_{13}\left(M_{V}^{2}\right)= & \left.J_{12}\left(M_{V}^{2}\right)\right|_{\hat{t} \leftrightarrow \hat{u}}, \\
J_{14 a}\left(M_{V}^{2}\right)= & D_{0}\left(p_{W}^{2}, m^{2}, 0, m^{2}, \hat{t}, \hat{u} ; M_{V}^{2}, M_{W}^{2}, m^{2}, m^{2}\right) \\
& -\frac{\hat{t} C_{0}\left(\hat{t}, 0, m^{2} ; M_{V}^{2}, m^{2}, m^{2}\right)+\hat{u} C_{0}\left(\hat{u}, 0, m^{2} ; M_{W}^{2}, m^{2}, m^{2}\right)}{\hat{t} \hat{u}-\hat{t} M_{W}^{2}-\hat{u} M_{V}^{2}}, \\
J_{14 b}\left(M_{V}^{2}\right)= & \left.J_{14 a}\left(M_{V}^{2}\right)\right|_{M_{W}^{2} \leftrightarrow M_{V}^{2}}=\left.J_{14 a}\left(M_{V}^{2}\right)\right|_{\hat{t} \leftrightarrow \hat{u}} \cdot
\end{aligned}
$$

For non-vanishing $M_{V}$ and $W$-boson transverse momentum, the functions $J_{12}-J_{14 b}$ are finite. The fact that the scalar four-point functions in (134) appear always in combination with three-point functions is due to the cancellation of the collinear singularities that are associated with the $g q \bar{q}$ vertex [9]. Although these singularities are present in individual $D_{0}$ and $C_{0}$ functions, they always cancel in the complete result for box diagrams. 


\section{Infrared singularities}

The scalar integrals $J_{i}\left(M_{V}^{2}\right)$ in Appendix C contain soft and collinear singularities that appear when $M_{V}=M_{A} \rightarrow 0$ and $m \rightarrow 0$. As discussed in Sect. 3.4, these integrals are split into IR-singular (IR) and IR-finite (fin) parts,

$$
J_{i}\left(M_{A}^{2}\right)=J_{i}^{\mathrm{IR}}+J_{i}^{\mathrm{fin}} .
$$

The IR-singular parts depend on the scheme adopted to regularize soft and collinear singularities. The IR-finite parts are scheme independent and free from soft-collinear singularities, but can contain ultraviolet poles.

Let us start with the two-point functions (132). Here only $J_{1 a}\left(M_{A}^{2}\right)$ gives rise to IR singularities. This integral is split into

$$
\begin{aligned}
J_{1 a, \mathrm{MR}}^{\mathrm{IR}} & =-\ln \left(\frac{m^{2}}{M_{W}^{2}}\right)+1, \\
J_{1 a, \mathrm{DR}}^{\mathrm{IR}} & =-\left(\frac{4 \pi \mu^{2}}{M_{W}^{2}}\right)^{\varepsilon} \frac{\Gamma(1+\varepsilon)}{\varepsilon}-1, \\
J_{1 a}^{\mathrm{fin}} & =\left(\frac{4 \pi \mu^{2}}{M_{W}^{2}}\right)^{\varepsilon} \frac{\Gamma(1+\varepsilon)}{\varepsilon}+1 .
\end{aligned}
$$

We note that within dimensional regularization the UV and IR singularities cancel each other and the massless two-point function vanishes, $J_{1 a, \mathrm{DR}}^{\mathrm{IR}}+J_{1 a}^{\mathrm{fin}}=0$. The three-point functions $J_{9 b}\left(M_{A}^{2}\right)$ and $J_{11 b}\left(M_{A}^{2}\right)$ are free from IR singularities and the singularities originating from $J_{8}\left(M_{A}^{2}\right)$ and $J_{10}\left(M_{A}^{2}\right)$ do not need to be considered since the coefficients associated with these scalar integrals are of order $M_{A}^{2}$ (see Appendix E). The remaining three-point functions in (133) contain soft and collinear singularities. For them we find

$$
\begin{aligned}
J_{7, \mathrm{MR}}^{\mathrm{IR}} & =\frac{1}{\hat{s}}\left[-\frac{1}{2} \ln ^{2}\left(\frac{M_{W}^{2}}{m^{2}}\right)+\ln \left(\frac{M_{W}^{2}}{\lambda^{2}}\right) \ln \left(\frac{-\hat{s}}{m^{2}}\right)\right], \\
J_{7, \mathrm{DR}}^{\mathrm{IR}} & =\left(\frac{4 \pi \mu^{2}}{M_{W}^{2}}\right)^{\varepsilon} \frac{\Gamma(1+\varepsilon)}{\hat{s}}\left[\frac{1}{\varepsilon^{2}}-\frac{1}{\varepsilon} \ln \left(\frac{-\hat{s}}{M_{W}^{2}}\right)\right], \\
J_{7}^{\mathrm{fin}} & =\frac{1}{\hat{s}}\left[\frac{1}{2} \ln ^{2}\left(\frac{-\hat{s}}{M_{W}^{2}}\right)-\frac{\pi^{2}}{6}\right],
\end{aligned}
$$

and

$$
\begin{aligned}
J_{9 a, \mathrm{MR}}^{\mathrm{IR}}= & \frac{1}{\hat{u}-M_{W}^{2}}\left\{\frac{1}{2}\left[\ln \left(\frac{M_{W}^{2}}{\lambda^{2}}\right)-\frac{1}{2} \ln \left(\frac{M_{W}^{2}}{m^{2}}\right)\right] \ln \left(\frac{M_{W}^{2}}{m^{2}}\right)\right. \\
& \left.+\ln \left(\frac{M_{W}^{2}}{\lambda^{2}}\right) \ln \left(1-\frac{\hat{u}}{M_{W}^{2}}\right)\right\}, \\
J_{9 a, \mathrm{DR}}^{\mathrm{IR}}= & \left(\frac{4 \pi \mu^{2}}{M_{W}^{2}}\right)^{\varepsilon} \frac{\Gamma(1+\varepsilon)}{\hat{u}-M_{W}^{2}}\left[\frac{1}{2 \varepsilon^{2}}-\frac{1}{\varepsilon} \ln \left(1-\frac{\hat{u}}{M_{W}^{2}}\right)\right], \\
J_{9 a}^{\mathrm{fin}}= & \frac{1}{\hat{u}-M_{W}^{2}}\left[\ln ^{2}\left(1-\frac{\hat{u}}{M_{W}^{2}}\right)+\operatorname{Li}_{2}\left(\frac{\hat{u}}{M_{W}^{2}}\right)\right],
\end{aligned}
$$


where $\operatorname{Li}_{2}(x)=-\int_{0}^{x} \mathrm{~d} t \ln (1-t) / t$. The finite and singular parts for $J_{11 a}\left(M_{A}^{2}\right)=$ $\left.J_{9 a}\left(M_{A}^{2}\right)\right|_{\hat{u} \rightarrow \hat{t}}$ are constructed in the same way.

The singular parts of the subtracted four-point functions (134) can be related to the ones of the three-point functions,

$$
\begin{aligned}
J_{12}^{\mathrm{IR}} & =\left.J_{13}^{\mathrm{IR}}\right|_{\hat{t} \leftrightarrow \hat{u}}=\frac{1}{\hat{u}} J_{7}^{\mathrm{IR}}, \\
J_{14 a}^{\mathrm{IR}} & =\left.J_{14 b}^{\mathrm{IR}}\right|_{\hat{t} \leftrightarrow \hat{u}}=\frac{1}{\hat{t}} J_{9 a}^{\mathrm{IR}},
\end{aligned}
$$

in both regularization schemes. This implicitly defines the remainders as

$$
\begin{aligned}
& J_{12}^{\mathrm{fin}}=\left.J_{13}^{\mathrm{fin}}\right|_{\hat{t} \leftrightarrow \hat{u}}=J_{12}\left(M_{A}^{2}\right)-\frac{1}{\hat{u}} J_{7}^{\mathrm{IR}}, \\
& J_{14 a}^{\mathrm{fin}}=\left.J_{14 b}^{\mathrm{fin}}\right|_{\hat{t} \leftrightarrow \hat{u}}=J_{14 a}\left(M_{A}^{2}\right)-\frac{1}{\hat{t}} J_{9 a}^{\mathrm{IR}} .
\end{aligned}
$$

Using the explicit analytic expressions for the infrared singular four-point and threepoint functions [41, 42] we obtain

$$
\begin{aligned}
J_{12}^{\mathrm{fin}}= & \frac{1}{\hat{s} \hat{u}}\left[\frac{1}{2} \ln ^{2}\left(\frac{-\hat{s}}{M_{W}^{2}}\right)-\ln ^{2}\left(\frac{\hat{s}}{\hat{u}}\right)-2 \operatorname{Li}_{2}\left(1-\frac{M_{W}^{2}}{\hat{s}}\right)-2 \operatorname{Li}_{2}\left(1-\frac{M_{W}^{2}}{\hat{u}}\right)-\frac{\pi^{2}}{2}\right], \\
J_{14 a}^{\mathrm{fin}}= & \frac{1}{\hat{t}\left(\hat{u}-M_{W}^{2}\right)}\left[2 \ln \left(1-\frac{\hat{u}}{M_{W}^{2}}\right) \ln \left(\frac{-\hat{t}}{M_{W}^{2}}\right)-\frac{1}{2} \ln ^{2}\left(\frac{-\hat{t}}{M_{W}^{2}}\right)\right. \\
& \left.+\operatorname{Li}_{2}\left(\frac{\hat{u}}{M_{W}^{2}}\right)-\frac{\pi^{2}}{2}\right] .
\end{aligned}
$$

\section{E Explicit result for the virtual corrections}

In this appendix we present explicit analytic expression for the functions $H_{1}^{\mathrm{I}}\left(M_{V}^{2}\right)$ defined in (74). These functions describe the contribution of the unrenormalized Feynman diagrams of Fig. 2 to the unpolarized cross section. They consist of linear combinations of the scalar integrals defined in Appendix $\mathrm{C}$.

$$
H_{1}^{\mathrm{I}}\left(M_{V}^{2}\right)=\sum_{j} K_{j}^{\mathrm{I}}\left(M_{V}^{2}\right) \operatorname{Re}\left[J_{j}\left(M_{V}^{2}\right)\right] \quad \text { for } \quad \mathrm{I}=\mathrm{A}, \mathrm{N}, \mathrm{X}, \mathrm{Y} .
$$

The coefficients of the function $H_{1}^{\mathrm{A}}\left(M_{V}^{2}\right)$ read

$$
\begin{aligned}
K_{0}^{\mathrm{A}}\left(M_{V}^{2}\right)= & -\frac{4 \hat{s}^{2}+3\left(\hat{t}^{2}+\hat{u}^{2}\right)}{\hat{t} \hat{u}}+\hat{s}\left(\frac{1}{\hat{s}+\hat{t}}+\frac{1}{\hat{s}+\hat{u}}-\frac{5}{\hat{u}}-\frac{5}{\hat{t}}+\frac{4}{\hat{t}+\hat{u}}\right), \\
K_{1 a}^{\mathrm{A}}\left(M_{V}^{2}\right)= & M_{V}^{2}\left\{-\left[\frac{3 \hat{s}}{(\hat{s}+\hat{t})^{2}}+\frac{3 \hat{s}}{(\hat{s}+\hat{u})^{2}}\right]+\left(\frac{1}{\hat{s}+\hat{t}}+\frac{1}{\hat{s}+\hat{u}}\right)\right. \\
& \left.-2\left(\frac{\hat{s}+\hat{u}}{\hat{t}^{2}}+\frac{\hat{s}+\hat{t}}{\hat{u}^{2}}\right)+\frac{2 \hat{s}^{2}(2 \hat{s}+\hat{t}+\hat{u})}{\hat{t} \hat{u}(\hat{s}+\hat{t})(\hat{s}+\hat{u})}\right\}+4 \frac{(\hat{s}+\hat{t})^{2}+(\hat{s}+\hat{u})^{2}}{\hat{t} \hat{u}},
\end{aligned}
$$




$$
\begin{aligned}
& K_{1 b}^{\mathrm{A}}\left(M_{V}^{2}\right)=0, \\
& K_{2}^{\mathrm{A}}\left(M_{V}^{2}\right)=p_{W}^{2}\left[\frac{6 \hat{s} M_{V}^{2}}{(\hat{s}+\hat{t})^{3}}+\frac{6 \hat{s} M_{V}^{2}}{(\hat{s}+\hat{u})^{3}}+\frac{2 \hat{s} M_{V}^{2}}{(\hat{s}+\hat{t})^{2} \hat{u}}+\frac{2 \hat{s} M_{V}^{2}}{(\hat{s}+\hat{u})^{2} \hat{t}}+\frac{4(\hat{s}+\hat{t}+\hat{u})}{(\hat{t}+\hat{u})^{2}}-\frac{3}{\hat{t}}\right. \\
& \left.-\frac{3}{\hat{u}}+\frac{2 \hat{s}+\hat{t}-2 M_{V}^{2}}{(\hat{s}+\hat{t})^{2}}+\frac{2 \hat{s}+\hat{u}-2 M_{V}^{2}}{(\hat{s}+\hat{u})^{2}}-\frac{\hat{s}(2 \hat{s}+\hat{t}+\hat{u})\left(2 M_{V}^{2}+3 \hat{s}\right)}{\hat{t} \hat{u}(\hat{s}+\hat{t})(\hat{s}+\hat{u})}\right] \text {, } \\
& K_{3}^{\mathrm{A}}\left(M_{V}^{2}\right)=0 \text {, } \\
& K_{4}^{\mathrm{A}}\left(M_{V}^{2}\right)=-\frac{4 \hat{s}(\hat{s}+2 \hat{t}+2 \hat{u})}{(\hat{t}+\hat{u})^{2}}, \\
& K_{5 a}^{\mathrm{A}}\left(M_{V}^{2}\right)=-\frac{6 M_{V}^{2} \hat{s} \hat{u}}{(\hat{s}+\hat{t})^{3}}+\frac{M_{V}^{2}(2 \hat{u}-5 \hat{s})-\hat{s} \hat{u}}{(\hat{s}+\hat{t})^{2}}+\frac{2 M_{V}^{2}(\hat{s}+\hat{t}+\hat{u})}{\hat{u}^{2}}-\frac{M_{V}^{2}+4 \hat{s}+\hat{u}}{\hat{s}+\hat{t}}, \\
& K_{5 b}^{\mathrm{A}}\left(M_{V}^{2}\right)=0 \text {, } \\
& K_{6 a}^{\mathrm{A}}\left(M_{V}^{2}\right)=\left.K_{5 a}^{\mathrm{A}}\left(M_{V}^{2}\right)\right|_{\hat{t} \leftrightarrow \hat{u}}, \\
& K_{6 b}^{\mathrm{A}}\left(M_{V}^{2}\right)=0 \text {, } \\
& K_{7}^{\mathrm{A}}\left(M_{V}^{2}\right)=-\frac{\hat{s}}{\hat{t} \hat{u}}\left[2\left(\hat{s}+M_{V}^{2}\right)(\hat{t}+\hat{u})+\hat{t}^{2}+\hat{u}^{2}\right] \text {, } \\
& K_{8}^{\mathrm{A}}\left(M_{V}^{2}\right)=\frac{p_{W}^{2} M_{V}^{2}}{\hat{u}\left(\hat{u}-p_{W}^{2}\right)^{3}}\left[2 \hat{t} M_{V}^{2}(\hat{u}-\hat{s}-\hat{t})-4 p_{W}^{2} \hat{s}\left(\hat{s}+\hat{t}+M_{V}^{2}\right)\right], \\
& K_{9 a}^{\mathrm{A}}\left(M_{V}^{2}\right)=K_{9 b}^{\mathrm{A}}\left(M_{V}^{2}\right)=0, \\
& K_{10}^{\mathrm{A}}\left(M_{V}^{2}\right)=\left.K_{8}^{\mathrm{A}}\left(M_{V}^{2}\right)\right|_{\hat{t} \leftrightarrow \hat{u}}, \\
& K_{11 a}^{\mathrm{A}}\left(M_{V}^{2}\right)=K_{11 b}^{\mathrm{A}}\left(M_{V}^{2}\right)=0 \text {, } \\
& K_{12}^{\mathrm{A}}\left(M_{V}^{2}\right)=-\frac{M_{V}^{2}(\hat{t}+\hat{u})+\hat{s} \hat{u}}{\hat{t} \hat{u}}\left[2\left(\hat{s}+M_{V}^{2}\right)\left(\hat{s}+M_{V}^{2}+\hat{t}\right)+\hat{t}^{2}\right] \text {, } \\
& K_{13}^{\mathrm{A}}\left(M_{V}^{2}\right)=\left.K_{12}^{\mathrm{A}}\left(M_{V}^{2}\right)\right|_{\hat{t} \leftrightarrow \hat{u}}, \\
& K_{14 a}^{\mathrm{A}}\left(M_{V}^{2}\right)=K_{14 b}^{\mathrm{A}}\left(M_{V}^{2}\right)=0 \text {. }
\end{aligned}
$$

The only difference between $H_{1}^{\mathrm{A}}\left(M_{V}^{2}\right)$ and the equally named function in Ref. [9] is due to the fact that $H_{1}^{\mathrm{A}}\left(M_{V}^{2}\right)$ in Ref. [9] includes the contribution of the fermionic wave-function renormalization constants, which modify the coefficients $K_{0}^{\mathrm{A}}$ and $K_{1 a}^{\mathrm{A}}$ (see eqs. (54) and (55) in Ref. [9]).

For the coefficients of the function $H_{1}^{\mathrm{N}}\left(M_{V}^{2}\right)$ we obtain

$$
\begin{aligned}
K_{0}^{\mathrm{N}}\left(M_{V}^{2}\right)= & \frac{4 \hat{s}}{\hat{t} \hat{u}}(\hat{s}+\hat{t}+\hat{u})-2 \hat{s}\left(\frac{1}{\hat{s}+\hat{u}}+\frac{1}{\hat{s}+\hat{t}}+\frac{2}{\hat{t}+\hat{u}}\right)+2\left(\frac{\hat{t}}{\hat{u}}+\frac{\hat{u}}{\hat{t}}\right), \\
K_{1 a}^{\mathrm{N}}\left(M_{V}^{2}\right)= & -\frac{M_{V}^{2}}{2}\left\{\frac{4 \hat{s}}{\hat{\hat{t} \hat{u}}}+(\hat{t} \hat{u}-2 \hat{s}(\hat{s}+\hat{t}+\hat{u}))\left[\frac{1}{\hat{t}(\hat{s}+\hat{u})^{2}}+\frac{1}{\hat{u}(\hat{s}+\hat{t})^{2}}\right]\right\} \\
& -\frac{\hat{s}(4 \hat{s}+3 \hat{t}+3 \hat{u})+\hat{t}^{2}+\hat{u}^{2}}{\hat{t} \hat{u}},
\end{aligned}
$$




$$
\begin{aligned}
& K_{1 b}^{\mathrm{N}}\left(M_{V}^{2}\right)=-\left.K_{1 a}^{\mathrm{N}}\left(M_{V}^{2}\right)\right|_{M_{V}^{2} \leftrightarrow M_{W}^{2}}, \\
& K_{2}^{\mathrm{N}}\left(M_{V}^{2}\right)=-K_{2}^{\mathrm{A}}\left(M_{V}^{2}\right), \\
& K_{3}^{\mathrm{N}}\left(M_{V}^{2}\right)=\left(M_{W}^{2}+M_{V}^{2}\right)\left[\frac{3 \hat{s} \hat{t}}{(\hat{s}+\hat{u})^{3}}+\frac{3 \hat{s} \hat{u}}{(\hat{s}+\hat{t})^{3}}\right]-\frac{1}{\hat{t} \hat{u}}\left[\frac{1}{(\hat{s}+\hat{t})^{2}}+\frac{1}{(\hat{s}+\hat{u})^{2}}\right] \\
& \times\left\{\hat{s}^{4}-2 \hat{t}^{2} \hat{u}^{2}+\hat{s}^{2}(\hat{t}+\hat{u})(2 \hat{s}+\hat{t}+\hat{u})+\left(M_{W}^{2}+M_{V}^{2}\right)\right. \\
& \left.\times\left[\hat{s}^{2}(\hat{s}+\hat{t}+\hat{u})-\hat{t} \hat{u}(2 \hat{s}-\hat{t}-\hat{u})\right]\right\} \\
& K_{4}^{\mathrm{N}}\left(M_{V}^{2}\right)=-K_{4}^{\mathrm{A}}\left(M_{V}^{2}\right), \\
& K_{5 a}^{\mathrm{N}}\left(M_{V}^{2}\right)=\frac{2 \hat{s}(\hat{s}+\hat{t})-2 \hat{t} \hat{u}}{2(\hat{s}+\hat{t})^{2}}-\left[\frac{\hat{s}+\hat{t}}{\hat{u}}-\frac{2 \hat{s}}{\hat{s}+\hat{t}}-\frac{\hat{u}(2 \hat{s}+\hat{t})}{2(\hat{s}+\hat{t})^{2}}\right]-M_{V}^{2}\left[\frac{1}{\hat{u}}-\frac{2 \hat{s}+\hat{t}}{2(\hat{s}+\hat{t})^{2}}\right] \\
& +\left(2 M_{V}^{2}-M_{W}^{2}\right)\left[\frac{2 \hat{s}}{(\hat{s}+\hat{t})^{2}}+\frac{\hat{u}(2 \hat{s}-\hat{t})}{(\hat{s}+\hat{t})^{3}}\right] \text {, } \\
& K_{5 b}^{\mathrm{N}}\left(M_{V}^{2}\right)=\frac{2 \hat{s}(\hat{s}+\hat{t})-2 \hat{t} \hat{u}}{2(\hat{s}+\hat{t})^{2}}+\left[\frac{\hat{s}+\hat{t}}{\hat{u}}-\frac{2 \hat{s}}{\hat{s}+\hat{t}}-\frac{\hat{u}(2 \hat{s}+\hat{t})}{2(\hat{s}+\hat{t})^{2}}\right]+M_{W}^{2}\left[\frac{1}{\hat{u}}-\frac{2 \hat{s}+\hat{t}}{2(\hat{s}+\hat{t})^{2}}\right] \\
& -M_{V}^{2}\left[\frac{2 \hat{s}}{(\hat{s}+\hat{t})^{2}}+\frac{\hat{u}(2 \hat{s}-\hat{t})}{(\hat{s}+\hat{t})^{3}}\right] \text {, } \\
& K_{6 a}^{\mathrm{N}}\left(M_{V}^{2}\right)=\left.K_{5 a}^{\mathrm{N}}\left(M_{V}^{2}\right)\right|_{\hat{t} \leftrightarrow \hat{u}}, \\
& K_{6 b}^{\mathrm{N}}\left(M_{V}^{2}\right)=\left.K_{5 b}^{\mathrm{N}}\left(M_{V}^{2}\right)\right|_{\hat{t} \leftrightarrow \hat{u}}, \\
& K_{7}^{\mathrm{N}}\left(M_{V}^{2}\right)=-K_{7}^{\mathrm{A}}\left(M_{V}^{2}\right), \\
& K_{8}^{\mathrm{N}}\left(M_{V}^{2}\right)=-K_{8}^{\mathrm{A}}\left(M_{V}^{2}\right) \text {, } \\
& K_{9 a}^{\mathrm{N}}\left(M_{V}^{2}\right)=\hat{u}-\frac{\hat{s}^{2}-\hat{s} \hat{t}}{2 \hat{t}}+\frac{\hat{s}^{2}+\hat{s} \hat{t}}{2 \hat{u}}+\left(M_{W}^{2}+M_{V}^{2}\right)\left[\frac{2 \hat{s}^{2}+\hat{t} \hat{s}+\hat{t}^{2}}{2 \hat{t} \hat{u}}-\frac{\hat{t}-\hat{s}}{2 \hat{t}}\right] \\
& -\frac{2 M_{V}^{2} \hat{t} \hat{u}}{(\hat{s}+\hat{t})^{2}}+\frac{M_{V}^{2}}{\hat{s}+\hat{t}}\left[\frac{M_{W}^{2} \hat{s}^{2}}{\hat{t} \hat{u}}-\frac{M_{V}^{2} \hat{u}(2 \hat{s}-\hat{t})}{(\hat{s}+\hat{t})^{2}}-\frac{2\left(M_{W}^{2}+M_{V}^{2}\right) \hat{s}}{\hat{s}+\hat{t}}\right], \\
& K_{9 b}^{\mathrm{N}}\left(M_{V}^{2}\right)=\left.K_{9 a}^{\mathrm{N}}\left(M_{V}^{2}\right)\right|_{M_{V}^{2} \leftrightarrow M_{W}^{2}}, \\
& K_{10}^{\mathrm{N}}\left(M_{V}^{2}\right)=\left.K_{8}^{\mathrm{N}}\left(M_{V}^{2}\right)\right|_{\hat{t} \leftrightarrow \hat{u}}=-K_{10}^{\mathrm{A}}\left(M_{V}^{2}\right), \\
& K_{11 a}^{\mathrm{N}}\left(M_{V}^{2}\right)=\left.K_{9 a}^{\mathrm{N}}\left(M_{V}^{2}\right)\right|_{\hat{t} \leftrightarrow \hat{u}} \text {, } \\
& K_{11 b}^{\mathrm{N}}\left(M_{V}^{2}\right)=\left.K_{9 b}^{\mathrm{N}}\left(M_{V}^{2}\right)\right|_{\hat{t} \leftrightarrow \hat{u}} \text {, } \\
& K_{12}^{\mathrm{N}}\left(M_{V}^{2}\right)=-K_{12}^{\mathrm{A}}\left(M_{V}^{2}\right) \text {, } \\
& K_{13}^{\mathrm{N}}\left(M_{V}^{2}\right)=\left.K_{12}^{\mathrm{N}}\left(M_{V}^{2}\right)\right|_{\hat{t} \leftrightarrow \hat{u}}=-K_{13}^{\mathrm{A}}\left(M_{V}^{2}\right), \\
& K_{14 a}^{\mathrm{N}}\left(M_{V}^{2}\right)=\frac{M_{W}^{2} \hat{t}+M_{V}^{2} \hat{u}-\hat{t} \hat{u}}{2 \hat{t} \hat{u}}\left[2 M_{W}^{2} M_{V}^{2}+(2 \hat{s}+\hat{t}+\hat{u})\left(M_{W}^{2}+M_{V}^{2}\right)-2 \hat{t} \hat{u}\right.
\end{aligned}
$$




$$
\begin{gathered}
-\hat{s}(\hat{t}+\hat{u})], \\
K_{14 b}^{\mathrm{N}}\left(M_{V}^{2}\right)=\left.K_{14 a}^{\mathrm{N}}\left(M_{V}^{2}\right)\right|_{M_{V}^{2} \leftrightarrow M_{W}^{2}} .
\end{gathered}
$$

For $M_{V}=M_{W}$ the function $H_{1}^{\mathrm{N}}\left(M_{V}^{2}\right)$ is identical to the equally named function in Ref. [9].

The only non-vanishing coefficients of the function $H_{1}^{\mathrm{x}}\left(M_{V}^{2}\right)$ read

$$
\begin{aligned}
K_{0}^{\mathrm{X}}\left(M_{V}^{2}\right) & =-\frac{\hat{t}^{2}+\hat{u}^{2}+\hat{s}(\hat{t}+\hat{u})}{\hat{t} \hat{u}}, \\
K_{1 a}^{\mathrm{X}}\left(M_{V}^{2}\right) & =-\left[K_{5 a}^{\mathrm{X}}\left(M_{V}^{2}\right)+K_{6 a}^{\mathrm{X}}\left(M_{V}^{2}\right)\right], \\
K_{5 a}^{\mathrm{X}}\left(M_{V}^{2}\right) & =\frac{2\left(M_{V}^{2}-\hat{u}\right)(\hat{s}+\hat{t})}{\hat{u}^{2}}, \\
K_{6 a}^{\mathrm{X}}\left(M_{V}^{2}\right) & =\left.K_{5 a}^{\mathrm{X}}\left(M_{V}^{2}\right)\right|_{\hat{t} \leftrightarrow \hat{u}} .
\end{aligned}
$$

Finally, for $H_{1}^{\mathrm{Y}}\left(M_{V}^{2}\right)$ we obtain

$$
\begin{aligned}
K_{0}^{\mathrm{Y}}\left(M_{V}^{2}\right)= & \frac{(\hat{u}-\hat{t})(\hat{s}+\hat{t}+\hat{u})}{\hat{t} \hat{u}}, \\
K_{1 a}^{\mathrm{Y}}\left(M_{V}^{2}\right)= & M_{V}^{2}\left[\frac{2}{\hat{t}}+\frac{3 \hat{s}}{(\hat{s}+\hat{t})^{2}}-\frac{1}{\hat{s}+\hat{t}}-\frac{2}{\hat{u}}-\frac{3 \hat{s}}{(\hat{s}+\hat{u})^{2}}+\frac{1}{\hat{s}+\hat{u}}\right] \\
& +M_{V}^{2}\left[\frac{2(\hat{s}+\hat{u})}{\hat{t}^{2}}-\frac{2(\hat{s}+\hat{t})}{\hat{u}^{2}}\right], \\
K_{1 b}^{\mathrm{Y}}\left(M_{V}^{2}\right)= & -M_{W}^{2}\left[\frac{2}{\hat{t}}+\frac{3 \hat{s}}{(\hat{s}+\hat{t})^{2}}-\frac{1}{\hat{s}+\hat{t}}-\frac{2}{\hat{u}}-\frac{3 \hat{s}}{(\hat{s}+\hat{u})^{2}}+\frac{1}{\hat{s}+\hat{u}}\right] \\
& -\left[\frac{2(\hat{s}+\hat{u})}{\hat{t}}-\frac{2(\hat{s}+\hat{t})}{\hat{u}}\right], \\
K_{2}^{\mathrm{Y}}\left(M_{V}^{2}\right)= & \left.0, \quad-\hat{s}\left(2 \hat{t}^{2}-\hat{t} \hat{u}+2 \hat{u}^{2}\right)\right], \\
K_{3}^{\mathrm{Y}}\left(M_{V}^{2}\right)= & \frac{2\left(M_{W}^{2}-M_{V}^{2}\right)(\hat{t}-\hat{u})(\hat{s}+\hat{t}+\hat{u})}{(\hat{s}+\hat{t})^{3}(\hat{s}+\hat{u})^{3}}\left[-7 \hat{s}^{3}+\left(\hat{t} \hat{u}-6 \hat{s}^{2}\right)(\hat{t}+\hat{u})\right. \\
K_{4}^{\mathrm{Y}}\left(M_{V}^{2}\right)= & 0, \quad-\frac{2 \hat{s}^{2}+3 \hat{t} \hat{u}+2 \hat{s}(\hat{t}+\hat{u})}{(\hat{s}+\hat{t})^{2}}-M_{W}^{2}\left[\frac{4 \hat{s}}{(\hat{s}+\hat{t})^{2}}+\frac{2 \hat{u}(2 \hat{s}-\hat{t})}{(\hat{s}+\hat{t})^{3}}\right] \\
K_{5 a}^{\mathrm{Y}}\left(M_{V}^{2}\right)= & -M_{V}^{2}\left[\frac{2 \hat{s}+\hat{t}}{(\hat{s}+\hat{t})^{2}}-\frac{2}{\hat{u}}-\frac{2(\hat{s}+\hat{t})}{\hat{u}^{2}}\right], \\
K_{5 b}^{\mathrm{Y}}\left(M_{V}^{2}\right)= & \frac{2 \hat{s}^{2}+3 \hat{t} \hat{u}+2 \hat{s}(\hat{t}+\hat{u})}{(\hat{s}+\hat{t})^{2}}+M_{V}^{2}\left[\frac{4 \hat{s}}{(\hat{s}+\hat{t})^{2}}+\frac{2 \hat{u}(2 \hat{s}-\hat{t})}{(\hat{s}+\hat{t})^{3}}\right]
\end{aligned}
$$




$$
\begin{aligned}
&+M_{W}^{2}\left[\frac{2 \hat{s}+\hat{t}}{(\hat{s}+\hat{t})^{2}}-\frac{2}{\hat{u}}\right]-\frac{2(\hat{s}+\hat{t})}{\hat{u}}, \\
& K_{6 a}^{\mathrm{Y}}\left(M_{V}^{2}\right)=-\left.K_{5 a}^{\mathrm{Y}}\left(M_{V}^{2}\right)\right|_{\hat{t} \leftrightarrow \hat{u}}, \\
& K_{6 b}^{\mathrm{Y}}\left(M_{V}^{2}\right)=-\left.K_{5 b}^{\mathrm{Y}}\left(M_{V}^{2}\right)\right|_{\hat{t} \leftrightarrow \hat{u}}, \\
& K_{7}^{\mathrm{Y}}\left(M_{V}^{2}\right)= 0 \\
& K_{8}^{\mathrm{Y}}\left(M_{V}^{2}\right)= 0, \\
& K_{9 a}^{\mathrm{Y}}\left(M_{V}^{2}\right)= \frac{1}{(\hat{s}+\hat{t})^{3} \hat{t} \hat{u}}\left\{2 M_{V}^{4} \hat{t} \hat{u}\left[2 \hat{s}^{2}-\hat{t} \hat{u}+2 \hat{s}(\hat{t}+\hat{u})\right]\right. \\
&-M_{V}^{2}(\hat{s}+\hat{t})\left[(\hat{s}+\hat{t})^{2}\left(2 \hat{s}^{2}+\hat{s} \hat{t}+\hat{t}^{2}\right)+(\hat{s}-\hat{t})(\hat{s}+\hat{t})^{2} \hat{u}-4 \hat{t}^{2} \hat{u}^{2}\right. \\
&\left.+2 M_{W}^{2} \hat{s}(\hat{s}(\hat{s}+\hat{t})-2 \hat{t} \hat{u})\right]-(\hat{s}+\hat{t})^{3}\left[\hat{s}^{2}(\hat{t}-\hat{u})+2 \hat{t} \hat{u}^{2}\right. \\
&\left.\left.+\hat{s} \hat{t}(\hat{t}+\hat{u})+M_{W}^{2}\left(2 \hat{s}^{2}+\hat{t}(\hat{t}-\hat{u})+\hat{s}(\hat{t}+\hat{u})\right)\right]\right\} \\
& K_{9 b}^{\mathrm{Y}}\left(M_{V}^{2}\right)=-\left.K_{9 a}^{\mathrm{Y}}\left(M_{V}^{2}\right)\right|_{M_{V}^{2} \leftrightarrow M_{W}^{2}}, \\
& K_{10}^{\mathrm{Y}}\left(M_{V}^{2}\right)= 0, \\
& K_{11 a}^{\mathrm{Y}}\left(M_{V}^{2}\right)=-\left.K_{9 a}^{\mathrm{Y}}\left(M_{V}^{2}\right)\right|_{\hat{t} \leftrightarrow \hat{u}} \\
& K_{11 b}^{\mathrm{Y}}\left(M_{V}^{2}\right)=-\left.K_{9 b}^{\mathrm{Y}}\left(M_{V}^{2}\right)\right|_{\hat{t} \leftrightarrow \hat{u}}, \\
& K_{12}^{\mathrm{Y}}\left(M_{V}^{2}\right)= 0, \\
& K_{13}^{\mathrm{Y}}\left(M_{V}^{2}\right)= 0, \\
& K_{14 a}^{\mathrm{Y}}\left(M_{V}^{2}\right)=-2 K_{14 a}^{\mathrm{N}}\left(M_{V}^{2}\right), \\
& K_{14 b}^{\mathrm{Y}}\left(M_{V}^{2}\right)= 2 K_{14 b}^{\mathrm{Y}}\left(M_{V}^{2}\right)=-\left.K_{14 a}^{\mathrm{Y}}\left(M_{V}^{2}\right)\right|_{M_{V}^{2} \leftrightarrow M_{W}^{2}} \cdot \\
&(1 \\
&=
\end{aligned}
$$

\section{F Real corrections}

In Table 1 and Table 2 we list the dipoles that were used to calculate the subtraction terms in (91) for the massive regularization and in (109) for the dimensional regularization, respectively. We give references to the explicit formulae for the dipole terms and the phase-space mappings in the original paper [17] and [18, 19]. 


\begin{tabular}{|c|c|c|c|}
\hline Dipole & Type (emitter, spectator) & eq. no. & $\tilde{\Phi}_{2, n m}$ \\
\hline \hline$g_{a b, \tau}^{\text {sub }}$ & massless IS, massless IS & $(3.22)$ & $(3.25)-(3.27)$ \\
\hline$g_{a W, \tau}^{\text {sub }}$ & massless IS, massive FS & $(\mathrm{A} .1)$ & $(3.12)$ \\
\hline$g_{W a, \tau}^{\text {sub }}$ & massive FS, massless IS & $(\mathrm{A} .1)$ & $(3.12)$ \\
\hline$g_{a k, \tau}^{\text {sub }}$ & massless IS, massless FS & $(3.9)$ & $(3.12)$ \\
\hline$g_{k a, \tau}^{\text {sub }}$ & massless FS, massless IS & $(3.9)$ & $(3.12)$ \\
\hline$g_{k W, \tau}^{\text {sub }}$ & massless FS, massive FS & $(4.4)$ & $(4.5)$ \\
\hline$g_{W k, \tau}^{\text {sub }}$ & massive FS, massless FS & $(4.4)$ & $(4.5)$ \\
\hline
\end{tabular}

Table 1: Dipole subtraction terms from Ref. [17] used to calculate $\mathrm{M}_{\text {sub }}^{a b}$ in (91) for the massive regularization (IS = initial-state, $\mathrm{FS}=$ final-state).

\begin{tabular}{|c|c|c|c|}
\hline Dipole & Type (emitter, spectator) & eq. nos. & $\tilde{\Phi}_{2, n m}$ \\
\hline \hline $\mathcal{D}_{\text {QED }}^{a \gamma, b}$ & massless IS, massless IS & $\begin{array}{c}(5.136),(5.145) \\
\text { in Ref. [18] }\end{array}$ & $\begin{array}{c}(5.137),(5.139), \\
(5.140) \text { in Ref. [18] }\end{array}$ \\
\hline $\mathcal{D}_{W, \text { QED }}^{a \gamma}$ & massless IS, massive FS & $\begin{array}{c}(5.71),(5.81) \\
\text { in Ref. [19] }\end{array}$ & $\begin{array}{c}(5.73),(5.74) \\
\text { in Ref. [19] }\end{array}$ \\
\hline $\mathcal{D}_{\gamma W, \text { QED }}^{a}$ & massive FS, massless IS & $\begin{array}{c}(5.40),(5.50) \\
\text { in Ref. [19] }\end{array}$ & $\begin{array}{c}(5.42),(5.43) \\
\text { in Ref. [19] }\end{array}$ \\
\hline $\mathcal{D}_{k, \text { QED }}^{a \gamma}$ & massless IS, massless FS & $\begin{array}{c}(5.61),(5.65) \\
\text { in Ref. [18] }\end{array}$ & $\begin{array}{c}(5.62)-(5.64) \\
\text { in Ref. [18] }\end{array}$ \\
\hline $\mathcal{D}_{\gamma k, \text { QED }}^{a}$ & massless FS, massless IS & $(5.36),(5.39)$ & $(5.37),(5.38)$ \\
& & in Ref. [18] & in Ref. [18] \\
\hline $\mathcal{D}_{\gamma k, W, \text { QED }}$ & massless FS, massive FS & $(5.2),(5.16)$ & $(5.3),(5.7),(5.9)$ \\
& & in Ref. [19] & in Ref. [19] \\
\hline $\mathcal{D}_{\gamma W, k, \text { QED }}$ & massive FS, massless FS & $(5.2),(5.16)$ & $(5.3),(5.7),(5.9)$ \\
& & in Ref. [19] & in Ref. [19] \\
\hline
\end{tabular}

Table 2: Dipole expressions from Refs. [18, 19] used to calculate $M_{\text {sub }}^{a b}$ in (109) for the dimensional regularization. 


\section{References}

[1] G. Arnison et al. [UA1 Collaboration], Phys. Lett. B 122 (1983) 103; Phys. Lett. B 126 (1983) 398;

M. Banner et al. [UA2 Collaboration], Phys. Lett. B 122 (1983) 476;

P. Bagnaia et al. [UA2 Collaboration], Phys. Lett. B 129 (1983) 130.

[2] J. H. Kühn, A. A. Penin and V. A. Smirnov, Eur. Phys. J. C 17 (2000) 97 [hepph/9912503]; M. Ciafaloni, P. Ciafaloni and D. Comelli, Phys. Rev. Lett. 84 (2000) 4810 [hep-ph/0001142]; A. Denner and S. Pozzorini, Eur. Phys. J. C 18 (2001) 461 [hep-ph/0010201]; Eur. Phys. J. C 21 (2001) 63 [hep-ph/0104127]; J. H. Kühn et al., Nucl. Phys. B 616 (2001) 286 [hep-ph/0106298].

[3] B. Jantzen et al., Nucl. Phys. B 731 (2005) 188 [Erratum-ibid. B 752 (2006) 327] [hep-ph/0509157].

[4] V. S. Fadin et al., Phys. Rev. D 61 (2000) 094002 [hep-ph/9910338]; M. Melles, Phys. Rev. D 63 (2001) 034003 [hep-ph/0004056]; Phys. Rev. D 64 (2001) 054003 [hep-ph/0102097]; W. Beenakker and A. Werthenbach, Nucl. Phys. B 630 (2002) 3 [hep-ph/0112030]; A. Denner, M. Melles and S. Pozzorini, Nucl. Phys. B 662 (2003) 299 [hep-ph/0301241]; S. Pozzorini, Nucl. Phys. B 692 (2004) 135 hep-ph/0401087].

[5] S. Dittmaier and M. Krämer, Phys. Rev. D 65 (2002) 073007 hep-ph/0109062].

[6] U. Baur, O. Brein, W. Hollik, C. Schappacher and D. Wackeroth, Phys. Rev. D 65 (2002) 033007 hep-ph/0108274];

E. Accomando, A. Denner and S. Pozzorini, Phys. Rev. D 65 (2002) 073003 [hep-ph/0110114]; W. Hollik and C. Meier, Phys. Lett. B 590 (2004) 69 hep-ph/0402281; U. Baur and D. Wackeroth, Phys. Rev. D 70 (2004) 073015 hep-ph/0405191; E. Accomando, A. Denner and A. Kaiser, Nucl. Phys. B 706 (2005) 325 (hep-ph/0409247); E. Accomando, A. Denner and C. Meier, Eur. Phys. J. C 47 (2006) 125 [hep-ph/0509234]; J. H. Kühn, A. Scharf and P. Uwer, Eur. Phys. J. C 45 (2006) 139 [hep-ph/0508092]; hep-ph/0610335; S. Moretti, M. R. Nolten and D. A. Ross, Phys. Lett. B 639 (2006) 513 [hep-ph/0603083]; C. M. Carloni Calame, G. Montagna, O. Nicrosini and A. Vicini, JHEP 0612 (2006) 016 [hep-ph/0609170]; W. Bernreuther, M. Fuecker and Z. G. Si, Phys. Rev. D 74 (2006) 113005 [hep-ph/0610334].

[7] E. Maina, S. Moretti and D. A. Ross, Phys. Lett. B 593 (2004) 143 [Erratumibid. B 614 (2005) 216] hep-ph/0403050].

[8] J. H. Kühn, A. Kulesza, S. Pozzorini and M. Schulze, Phys. Lett. B 609 (2005) 277 [hep-ph/0408308]. 
[9] J. H. Kühn, A. Kulesza, S. Pozzorini and M. Schulze, Nucl. Phys. B 727 (2005) 368 hep-ph/0507178.

[10] J. H. Kühn, A. Kulesza, S. Pozzorini and M. Schulze, JHEP 0603 (2006) 059 hep-ph/0508253.

[11] U. Baur, Phys. Rev. D 75 (2007) 013005 hep-ph/0611241.

[12] A. Denner, B. Jantzen and S. Pozzorini, Nucl. Phys. B 761 (2007) 1 hep-ph/0608326.

[13] D. Wackeroth and W. Hollik, Phys. Rev. D 55 (1997) 6788 hep-ph/9606398]; U. Baur, S. Keller and D. Wackeroth, Phys. Rev. D 59 (1999) 013002 [hep-ph/9807417].

[14] A. Denner and S. Pozzorini, Eur. Phys. J. C 18 (2001) 461 [hep-ph/0010201]; Eur. Phys. J. C 21 (2001) 63 [hep-ph/0104127].

[15] A. Denner, M. Melles and S. Pozzorini, Nucl. Phys. B 662 (2003) 299 hep-ph/0301241.

[16] M. Melles, Phys. Rev. D 63 (2001) 034003 [hep-ph/0004056]; Phys. Rev. D 64 (2001) 014011 [hep-ph/0012157]; Phys. Rev. D 64 (2001) 054003 [hepph/0102097]; Phys. Rept. 375 (2003) 219 [hep-ph/0104232]; Eur. Phys. J. C 24 (2002) 193 [hep-ph/0108221].

[17] S. Dittmaier, Nucl. Phys. B 565 (2000) 69 [hep-ph/9904440].

[18] S. Catani and M. H. Seymour, Nucl. Phys. B 485 (1997) 291 [Erratum-ibid. B 510 (1998) 503] [hep-ph/9605323].

[19] S. Catani, S. Dittmaier, M. H. Seymour and Z. Trocsanyi, Nucl. Phys. B 627 (2002) 189 hep-ph/0201036.

[20] R. K. Ellis, G. Martinelli and R. Petronzio, Nucl. Phys. B 211 (1983) 106; P. B. Arnold and M. H. Reno, Nucl. Phys. B 319 (1989) 37 [Erratum-ibid. B 330 (1990) 284]; R. J. Gonsalves, J. Pawlowski and C. F. Wai, Phys. Rev. D 40 (1989) 2245; W. T. Giele, E. W. N. Glover and D. A. Kosower, Nucl. Phys. B 403 (1993) 633 [hep-ph/9302225]; J. Campbell, R. K. Ellis and D. L. Rainwater, Phys. Rev. D 68 (2003) 094021 hep-ph/0308195].

[21] J. H. Kühn, A. Kulesza, S. Pozzorini and M. Schulze, hep-ph/0703283.

[22] W. Hollik, T. Kasprzik, B.A. Kniehl, hep-ph/07072553.

[23] S. Pozzorini, doctoral thesis, Universität Zürich, 2001, hep-ph/0201077.

[24] G. Passarino and M. J. G. Veltman, Nucl. Phys. B 160 (1979) 151. 
[25] A. Denner, Fortsch. Phys. 41 (1993) 307.

[26] A. Sirlin, Phys. Rev. D 22 (1980) 971; W. J. Marciano and A. Sirlin, Phys. Rev. D 22 (1980) 2695 [Erratum-ibid. D 31, 213 (1985)]; Nucl. Phys. B 189 (1981) 442 .

[27] M. Böhm, A. Denner and H. Joos, "Gauge theories of the strong and electroweak interaction," Stuttgart, Germany: Teubner (2001) 784 p.

[28] M. Roth and A. Denner, Nucl. Phys. B 479 (1996) 495 [hep-ph/9605420].

[29] The expressions for the functions $H_{\mathrm{r}}^{i, \bar{q} q^{\prime}}$ in (88) are available from the authors upon request.

[30] Wolfram Research, Inc., Mathematica, Version 4.2, Champaign, IL (2002).

[31] G. J. van Oldenborgh, Comput. Phys. Commun. 66 (1991) 1.

[32] T. Stelzer and W. F. Long, Comput. Phys. Commun. 81 (1994) 357.

[33] G. P. Lepage, Cornell preprint CLNS 80-447.

[34] S. Eidelman et al. [Particle Data Group], Phys. Lett. B 592 (2004) 1.

[35] A. D. Martin, R. G. Roberts, W. J. Stirling and R. S. Thorne, Phys. Lett. B 531 (2002) 216 hep-ph/0201127.

[36] A. D. Martin, R. G. Roberts, W. J. Stirling and R. S. Thorne, Eur. Phys. J. C 39 (2005) 155 hep-ph/0411040].

[37] M. Roth and S. Weinzierl, Phys. Lett. B 590 (2004) 190 hep-ph/0403200.

[38] V. A. Mitsou, hep-ph/0004161.

[39] R. Dixon, presentation at the Fermilab Users' Meeting, Batavia, IL, June 2007. http://www.fnal.gov/orgs/fermilab_users_org/users_mtg/2007/agenda-for-web.htm

[40] R. Mertig, M. Böhm and A. Denner, Comput. Phys. Commun. 64 (1991) 345; http://www.feyncalc.org/.

[41] W. Beenakker and A. Denner, Nucl. Phys. B 338 (1990) 349.

[42] S. Dittmaier, Nucl. Phys. B 675 (2003) 447 hep-ph/0308246.

[43] E. W. N. Glover and A. G. Morgan, Z. Phys. C 62 (1994) 311.

[44] D. Buskulic et al. [ALEPH Collaboration], Z. Phys. C 69 (1996) 365. 
[45] L. Bourhis, M. Fontannaz and J. P. Guillet, Eur. Phys. J. C 2 (1998) 529 [hep-ph/9704447].

[46] M. Fontannaz, J. P. Guillet and G. Heinrich, Eur. Phys. J. C 21 (2001) 303 hep-ph/0105121.

[47] M. Klasen, Rev. Mod. Phys. 74 (2002) 1221 [hep-ph/0206169].

[48] A. Gehrmann-De Ridder, T. Gehrmann and E. Poulsen, Eur. Phys. J. C 47 (2006) 395 [hep-ph/0604030]. 